\title{
A Theoretical Approach to Pseudogap and Superconducting Transitions in Hole-Doped Cuprates
}

\author{
Partha Goswami \\ Physics Department, Deshbandhu College, Kalkaji, New Delhi 110019, India \\ Correspondence should be addressed to Partha Goswami; physics_goswami@yahoo.co.in
}

Received 7 August 2012; Accepted 27 August 2012

Academic Editors: H. Eisaki, A. A. Kordyuk, and T. Prokscha

Copyright (C) 2013 Partha Goswami. This is an open access article distributed under the Creative Commons Attribution License, which permits unrestricted use, distribution, and reproduction in any medium, provided the original work is properly cited.

\begin{abstract}
We consider a two-dimensional fermion system on a square lattice described by a mean-field Hamiltonian involving the singlet iddensity wave (DDW) order, assumed to correspond to the pseudo-gap (PG) state, favored by the electronic repulsion and the coexisting $d$-wave superconductivity (DSC) driven by an assumed attractive interaction within the BCS framework. Whereas the single-particle excitation spectrum of the pure DDW state consists of the fermionic particles and holes over the reasonably conducting background, the coexisting states corresponds to Bogoliubov quasi-particles in the background of the delocalized Cooper pairs in the momentum space. We find that the two gaps in the single-particle excitation spectrum corresponding to PG and DSC, respectively, are distinct and do not merge into one "quadrature" gap if the nesting property of the normal state dispersion is absent. We show that the PG and DSC are representing two competing orders as the former brings about a depletion of the spectral weight available for pairing in the anti-nodal region of momentum space where the superconducting gap is supposed to be the largest. This indicates that the PG state perhaps could not be linked to a preformed pairing scenario. We also show the depletion of the spectral weight below $T_{c}$ at energies larger than the gap amplitude. This is an important hallmark of the strong coupling superconductivity.
\end{abstract}

\section{Introduction}

A general consensus among the condensed matter physics community regarding the existence of the pseudogap (PG) phase in underdoped high $T_{c}$ superconductors has emerged after nearly a decade and half of the intensive theoretical and experimental studies [1-27]. However, regarding the origin of the PG and its relation with superconductivity (SC), there are divergent views. The interpretations run from descriptions where the PG is regarded as a superconducting precursor state involving incoherent electron-electron pairings above $T_{c}$ [1-7] with particle-hole symmetry of the SC state preserved to others where the PG, distinct from SC, corresponds to an ordered state with particle-hole asymmetry and both the phases compete [8-12]. In the former description, the preformed pairs appear at relatively high temperatures $T^{*} \sim 200 \mathrm{~K}$ compared to $T_{c}$ and one views $T^{*}$ as a "crossover" temperature, rather than a sharp phase transition. The origin of these preformed pairs is not fully known. They are supposed to arise from the attractive interaction which drives the superconductivity [21].

Our view regarding the origin of the PG is, however, centered around the simple paradigm that PG corresponds to $i d$-density wave ordering [9-11] (or its more complex variant, namely, $d+i d$ density wave ordering $[22,23,28-$ $30])$, at the antiferromagnetic wave vector $\mathbf{Q}=(\pi, \pi)$. Starting with a two-dimensional fermion system on a square lattice described by a mean-field Hamiltonian involving the singlet $i d$-density wave (DDW) order $\left(i \Delta_{k}=\left(i \Delta_{0}(T) / 2\right)\right.$ $\left.\left(\cos k_{x} a-\cos k_{y} a\right)\right)$ at the wave vector $\mathbf{Q}=(\pi, \pi)$, we intend to show that, for a transition to the PG state, upon lowering the temperature from $T>T^{*}$ to $T<T^{*}$ at a fixed doping level $(\sim 10 \%)$ on the underdoped side (see Figure 1), the entropy difference $\Delta S\left(=S_{\mathrm{PG}}-S_{\text {Normal }}\right)$ between the PG and normal paramagnetic states is negative. As an ordered state is expected to have lower entropy, there is nothing unusual about it. There appears to be no discontinuity in entropy at $T^{*}$ during this passage. There is no discontinuity in the electronic specific heat $C_{\mathrm{el}}$ either. This 


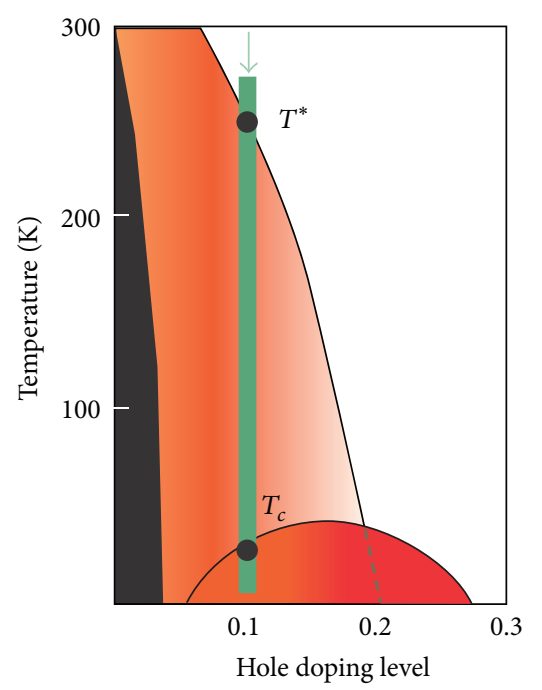

FIgURE 1: A generic phase diagram of the hole-doped cuprates in the doping level $(x)$-temperature $(T)$ plane. The antiferromagnetic phase corresponds to the blackened region in the far left. While the lightly red region to its right is the pseudogap (PG) state, the near-semicircular red region corresponds to the superconducting (SC) phase. The curves $T^{*}(x)$ and $T_{c}(x)$, respectively, correspond to the PG transition (from the paramagnetic insulating phase) and the SC transition. The vertical, thick green line within the SC phase corresponds to the region investigated which is a part of the superconducting DDW region.

is due to the fact that (high-energy) electronic excitations, responsible for the contributions towards the entropy and the specific heat, along the antinodal directions of momentum space where the pseudogap are maximal become scattered with shorter lifetimes (compared to hole excitations) and therefore unable to show any striking feature in $C_{\mathrm{el}}$ at $T^{*}$. The outcome of the calculation of in-plane quasiparticle thermal conductivity $\alpha_{x x}$ in both the phases via the Boltzmann equation in the relaxation-time approximation shows that there is a mild discontinuity in $\alpha_{x x}$ at $T^{*}: \alpha_{x x}$ for $t=$ $\left(T-T^{*}\right) / T^{*} \rightarrow 0_{-}$is found to be higher than $\alpha_{x x}$ for $t \rightarrow 0_{+}$. This is an unusual feature. We needed to solve the pseudogap equation and the equation to determine the chemical potential $(\mu)$ consistently in order to calculate $\alpha_{x x}$. We pinned $\mu$ close to van Hove singularities (vHSs) of the normal state dispersion $\varepsilon_{\mathbf{k}}$ (involving the first, the second, and third neighbor hoppings) in an effort to do so. We have rendered all quantities, such as $\alpha_{x x}$, dimensionless expressing $\varepsilon_{\mathbf{k}}$, and energy gaps, in units of the first neighbor hopping. The origin of the above-mentioned discontinuity in $\alpha_{x x}$ is found to rest on the fact that the PG phase has nodes in the gap function resulting in the longer-lived excitation of singlefermion states with negligible energies even down to very low temperature. These low-lying excitations have significant contribution in $\alpha_{x x}$. In fact, the entire bunch of energy states corresponding to the Fermi arc are the contributors. We find that the in-plane (dimensionless) longitudinal electrical conductivity in the PG phase $\sigma_{x x}$ is significantly greater than 1. As regards the Hall conductivities (electrical $\left(\sigma_{x y}\right)$ and thermal $\left.\left(\alpha_{x y}\right)\right)$, we found them nearly constant and close to

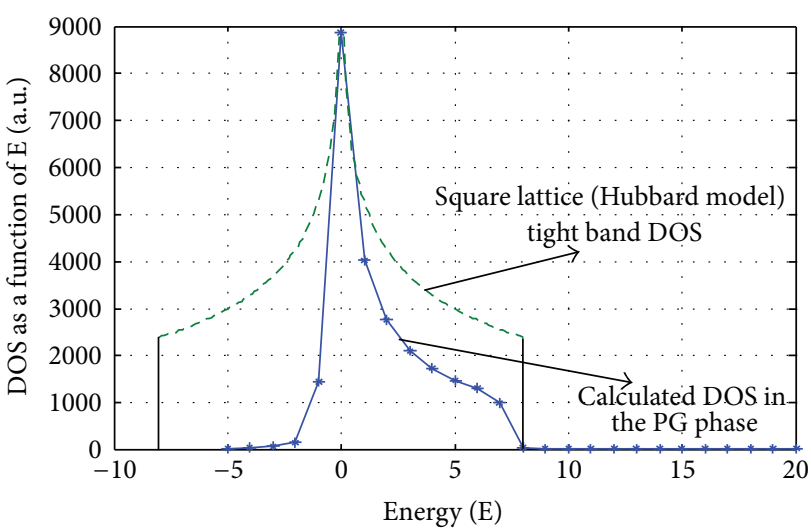

FIgURE 2: The 2D plot of the spectral function $A(\omega)=$ $\int d(\mathbf{k} a) A(\mathbf{k}, \omega)=\int_{-\pi}^{+\pi} d\left(k_{x} a\right) / 2 \pi \int_{-\pi}^{+\pi} d\left(k_{y} a\right) / 2 \pi A(\mathbf{k}, \omega)$ as a function of $\omega$, with a cusp at $\omega=0$. The negative $\omega$ states by and large correspond to k's close to $\Gamma$-point whereas positive $\omega$ states are close to $( \pm \pi, \pm \pi)$. For the comparison purpose, we have also plotted the square lattice tight band DOS in the Hubbard model which clearly shows vHS at $\omega=0$.

unity for all the temperatures considered between $T_{c}$ and $T^{*}$. Thus, for the in-plane electrical conductivities, the Hall angle $\tan \theta_{H} \equiv \sigma_{x y} / \sigma_{x x} \ll 1$. These results show reasonably conducting character of the PG state as has been reported by Levchenko et al. [13]. These authors have found the in-plane electrical and thermal conductivities are metal-like, while the $c$-axis resistivity and the Hall number are insulator-like in the pseudogap phase of the cuprates.

As regards $d$-wave superconductivity (DSC), we model the effective two-particle pairing interaction $V\left(\mathbf{k}, \mathbf{k}^{\prime}\right)$ in the singlet pairing channel by suitable function of the form $-\left|V_{1}\right|\left(\cos k_{x} a-\cos k_{y} a\right)\left(\cos k_{x}^{\prime} a-\cos k_{y}^{\prime} a\right)$, where $V_{1}$ is the coupling strength (model parameter). We assume implicitly that this unconventional superconductivity is initiated by the strongly coupled bosonic modes, such as those corresponding to the electron spin fluctuations (proximity to an antiferromagnetic phase raises the possibility of spinfluctuation-mediated pairing), leading to singlet pairing and concomitant kinetic energy reduction of the nodal quasiparticles at the pairing temperature $T_{c}$ which ultimately generates sufficiently high "quantum pressure" due to the temperature reduction to make the entire system undergo further lowering of kinetic energy and the free energy at $T<T_{c}$. Whereas the single-particle excitation spectrum of the pure DDW state consists of the fermionic particles and holes over the insulating background, the coexisting (CXS) DDW and DSC state corresponds to Bogoliubov quasiparticles (or "bogolons") in the background of the delocalized Cooper pairs in the momentum space. We find that the single-particle excitation spectrum $E_{\mathbf{k}}\left(T<T_{c}\right)=$ $\pm E^{(U, L)}(\mathbf{k}), E_{\mathbf{k}}^{(U, L) 2}=(1 / 2)\left[\left(\xi_{\mathbf{k}}^{2}+\xi_{\mathbf{k}+\mathbf{Q}}^{2}+2 D_{\text {eff }}(\mathbf{k})^{2}\right) \pm\right.$ $p(\mathbf{k})]$, where $p(\mathbf{k})=\left[\left(\xi_{\mathbf{k}}^{2}-\xi_{\mathbf{k}+\mathbf{Q}}^{2}\right)^{2}+4 \Delta_{\mathbf{k}}^{2}\left(\xi_{\mathbf{k}}+\xi_{\mathbf{k}+\mathbf{Q}}\right)^{2}\right]^{1 / 2}$, $D_{\text {eff }}(\mathbf{k})^{2}=\left[\Delta_{\mathbf{k}}^{2}+\Delta_{\mathbf{k}}^{(\mathrm{sc}) 2}\right]$, and $\xi_{\mathbf{k}}=\varepsilon_{\mathbf{k}}-\mu$. The symbol $\varepsilon_{\mathbf{k}}$ stands for the normal state dispersion and $\mu$ for 
the chemical potential of the fermion number. In our scheme, as already mentioned, the Fermi level is pinned at the Van Hove singularity of the dispersion $\varepsilon_{\mathbf{k}}$ involving the first, the second, and the third neighbor hoppings plus a constant term. All energies are expressed in units of the first neighbor hopping. With all these paraphernalia, there are only two energy gaps $i \Delta_{\mathbf{k}}$ and $\Delta_{\mathbf{k}}^{(\mathrm{sc})}$ corresponding to PG and DSC, respectively, and two distinct quasiparticle dynamics in our formulation of the problem. The second neighbor hopping in the dispersion, which is known to be important for cuprates [26] and frustrates the kinetic energy of electrons, leads to Fermi surface sheets being not connected by $\mathbf{Q}=(\pi, \pi)$ (nonnesting property). One may notice that the two gaps in the excitation spectrum are distinct and do not merge into one "quadrature" gap $\left(D_{\text {eff }}(\mathbf{k})^{2}=\left[\Delta_{\mathbf{k}}^{2}+\Delta_{\mathbf{k}}^{(\mathrm{sc}) 2}\right]\right)$ if the nesting property, $\varepsilon_{\mathbf{k}}=-\varepsilon_{\mathbf{k}+\mathbf{Q}}$, of the dispersion is absent; for the nested situation we do obtain such a merger yielding $E_{\mathbf{k}}^{(U)}=E_{\mathbf{k}}^{(L)}=\left(\xi_{\mathbf{k}}^{2}+D_{\text {eff }}(\mathbf{k})^{2}\right)^{1 / 2}$. As we shall see below (9), the nonnesting property of the dispersion is one of the important requirements for the onset of pure DDW ordering. To explain a little more, we note that the nesting is a meaningful phenomenon for an interacting system when we have a Fermi liquid (FL) description for the system. For interacting systems, all many-body effects are lumped into the self-energy $(\Sigma)$ part which is generally $\mathbf{k}$-independent. The $\operatorname{Re} \Sigma$ changes the quasiparticle dispersion away from the one corresponding to the noninteracting case whereas $\operatorname{Im} \Sigma$ gives the quasiparticle lifetime. The simplest example is the one corresponding to the repulsive Hubbard model $(U>$ 0 ) on a two-dimensional tight-binding square (bipartite) lattice. The kinetic energy connects only one sublattice to the other. The single-particle eigenstates for the noninteracting case have energies $\varepsilon_{\mathbf{k}}=\left\{-2 t_{1}\left(\cos \left(k_{x} a\right)+\cos \left(k_{y} a\right)\right)\right\}$ here. When fermions are poured into such a band, if initially the Fermi surface is circular that is, free-electron-like, at halffilling becomes a perfect square. The Fermi surface (FS) is nested with $\mathbf{Q}=( \pm \pi, \pm \pi)$ only at half-filling; close to halffilling the nesting is approximate. The density of states (DOS) displays nesting singularity. Upon inclusion of $U>0$, say, at the Hartree-Fock level, we obtain fermionic quasiparticles with reasonable $\mathrm{FL}$ description. In the $2 \mathrm{D}$ system under consideration in this communication, the FS nested with $\mathbf{Q}=$ $( \pm \pi, \pm \pi)$ in the pure DDW state also presents a particularly striking though untenable situation with single semimetallic band. In order to present a suitable description of cuprates, we thus have to turn our attention to nonnested dispersion (NND). However, the kinetic energy and DOS do not display the same behavior as in the $2 \mathrm{D}$ Hubbard model with this type of dispersion due to the second neighbor hopping. As we shall see below, the specific heat $C_{\mathrm{el}}$ shows anomalous temperature dependence, a typical non-Fermi liquid (NFL) feature, with the onset of DDW ordering. We thus note that nonnested dispersion and NFL behavior are perhaps as deeply connected as the nesting and the Fermi liquid behavior are for $2 \mathrm{D}$ systems.

The particle-hole asymmetry in the single-particle excitation spectrum (SPES) of the pure DDW state with NND is also reflected in the coexisting DDW and DSC states

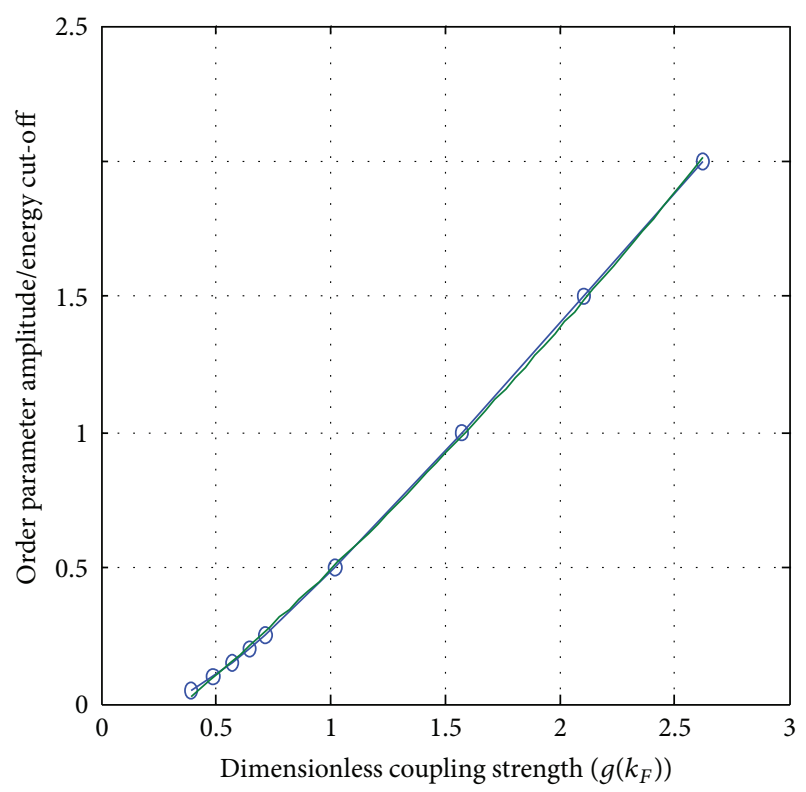

Figure 3: The 2D plot of $\left(\Delta_{0} / \hbar \omega_{c}\right)$ as a function of the dimensionless coupling strength $g\left(k_{F}\right)$ and second-degree polynomial fit.

(see Figure 4(c)) though the latter is characterized by the Bogoluibov quasiparticle bands-a prominent fingerprint of superconductivity. These results are qualitatively the same as those obtained by Hashimoto and his coworkers [24]. The particle-hole asymmetry in the CXS is an indication of the interplay of the two orderings. Obviously enough, the second neighbor hopping is partly responsible for this. We shall show that the pseudogap and high temperature superconductivity are representing two competing orders as the former brings about a depletion of the spectral weight $(\mathrm{SW}(T))$ available for pairing in the antinodal region of momentum space where the superconducting gap is supposed to be the largest. This indicates that the PG state could not be linked to a preformed pairing scenario. Furthermore, there is depletion of the spectral weight below $T_{c}$ at energies larger than the energy gap. We show this result analytically for the coexistent states calculating SW $(T)$ within the BCS framework for a two-dimensional fermion system on a square lattice starting with a Hamiltonian $H$ corresponding to the $i d$-density wave $(\mathrm{DDW})$ order plus the superconducting pairing $\Delta_{k}^{(\mathrm{sc})}=$ $\sum_{k^{\prime}, \sigma} V\left(\mathbf{k}, \mathbf{k}^{\prime}\right)\left\langle d_{-k^{\prime},-\sigma} d_{k^{\prime}, \sigma}\right\rangle$. This is a prominent spectroscopic evidence for the strong coupling superconductivity observed by Kaminski et al. [31].

The paper is organized as follows. In Section 2, we discuss the particle-hole asymmetry aspect of SPES in the coexistent DDW and DSC states. In Section 3, we derive expressions for the thermodynamic potential and entropy and exploit the latter for the estimation of the pseudogap transition temperature $T^{*}$. The electronic specific heat is shown to display anomalous temperature dependence. We also calculate the quasiparticle thermal conductivity $\alpha_{x x}$ in the normal and pseudogap phases via the Boltzmann 


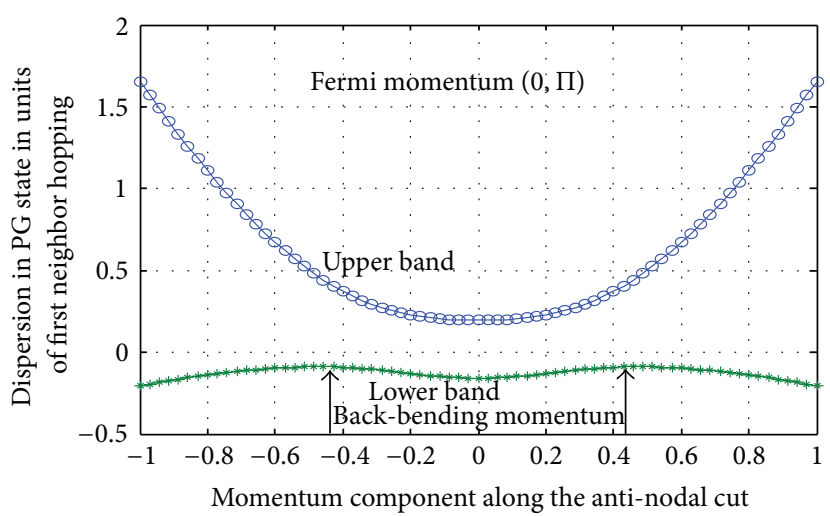

(a)

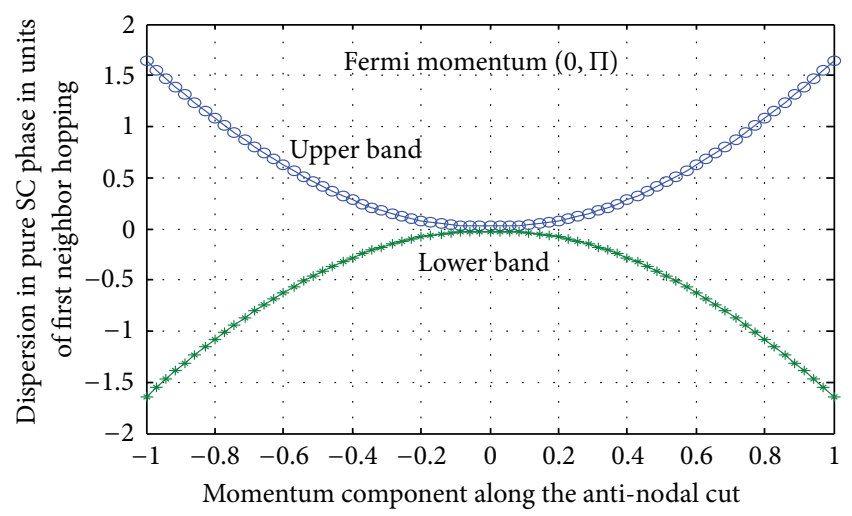

(b)

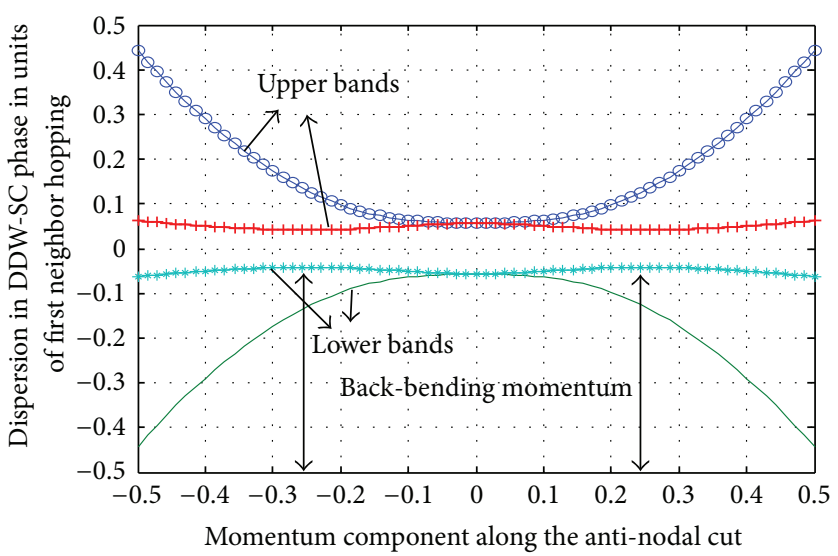

(c)

Figure 4: (a) A plot of the upper and the lower bands $\varepsilon_{k}^{(U, L)}$ in the pure DDW state (doping level 9.94\%) along the antinodal cut (- $\pi, \pi$ )$(0, \pi)-(\pi, \pi)$. The back-bending (or saturation) momentum of the dispersion $\varepsilon_{k}^{(L)}$ and the Fermi momentum $\left(k_{F}\right)$ are indicated. The numerical values, in the units of the first neighbor hopping $t_{1}$, are $\left(\mu / t_{1}\right)=-0.0183$, the PG gap amplitude $\left(\Delta_{0}^{(\mathrm{pg})} / t_{1}\right)=0.0610$, and the SC gap amplitude $\left(\Delta_{0}^{(\mathrm{sc})} / t_{1}\right)=0$. The hopping parameters are $\left(t_{2} / t_{1}\right)=0.3925$ and $\left(t_{3} / t_{1}\right)=0.0005$. The DDW ordering leads to pining of the Fermi level close to, but not precisely at, the Van Hove singularity. (b) A plot of the upper and the lower bands $\pm \dot{\varepsilon}(\mathbf{k})$ in the pure SC state along the antinodal cut $(-\pi, \pi)-(0, \pi)-(\pi, \pi)$ indicating perfect electron-hole symmetry (both the upper and lower bands are parabolic). The numerical value of the SC gap amplitude $\left(\Delta_{0}^{(\mathrm{sc})} / t_{1}\right)=0.0150$. The hopping parameters values and $\left(\mu / t_{1}\right)$ are the same as above. (c) A plot of the upper bands $+E_{k}^{(U, L)}$ and the lower bands $-E_{k}^{(U, L)}$ in the DDW-DSC state along the antinodal cut $(-\pi, \pi)-(0, \pi)-(\pi, \pi)$. The back-bending (or saturation) momentum of the dispersion $-E_{k}^{(L)}$ is indicated by double-headed arrows. This shoulder-type feature also exists in the experimental data of Hashimoto et al. [24]. Evidently, there are four quasiparticle bands (shown as a function of momentum ( $k$ ) along the antinodal cut) with two positioned at negative energy and two at positive energy for the Fermi energy taken as zero. The parameter values used are $\left(\mu / t_{1}\right)=-0.0018$, the PG gap amplitude $\left(\Delta_{0}^{(\mathrm{pg})} / t_{1}\right)=0.024$, and the SC gap amplitude $\left(\Delta_{0}^{(\mathrm{sc})} / t_{1}\right)=0.015$. The hopping parameters are $\left(t_{2} / t_{1}\right)=0.3913$ and $\left(t_{3} / t_{1}\right)=0.0005$.

equation in the relaxation-time approximation. The observed mild discontinuity in $\alpha_{x x}$ at $T^{*}$ indicates that the passage of the system from the normal to the PG state is a nonsharp thermal phase transition. The optical conductivity or the spectral weight occupies the centre-stage [32-40] in determining whether the pseudogap and high temperature superconductivity are representing two competing or cooperating orders. In Section 4, we discuss this issue in detail and conclude that these are competing orders. Besides, the calculation/plot of the integral

$$
F(\omega)=\int_{-\pi}^{+\pi} \frac{d\left(k_{x} a\right)}{2 \pi} \int_{-\pi}^{+\pi} \frac{d\left(k_{y} a\right)}{2 \pi} \sum_{\alpha} A(\mathbf{k}, \omega) \varepsilon_{\mathbf{k}}^{(x x)} n_{k, \alpha}(T),
$$


where the subscript " $\alpha$ " stands for the conduction band, $A(\mathbf{k}, \omega)$ is the density of states (DOSs) or spectral density (SD), $\varepsilon_{\mathbf{k}}^{(x x)}$ is the second derivative with respect to $k_{x}$ of dispersion $\varepsilon_{\mathbf{k}}$, and $n_{k, \alpha}(T)$ is the momentum distribution function at a temperature $T<T_{c}$, as a function of energy $(\omega)$ in units of the first neighbor hopping is obtained as a decreasing function for energy larger than that corresponding to the SC gap amplitude. This is an important hallmark of the strong coupling superconductivity. The paper ends in Section 5 with the concluding remarks.

\section{Bogoluibov Quasiparticle Bands}

In the second quantized notation, the Hamiltonian to deal with the id-density wave (DDW) order at the antiferromagnetic wave vector $\mathbf{Q}=(\pi, \pi)$ plus the $d$-wave superconductivity can be expressed as

$$
\begin{aligned}
H= & \sum_{k, \sigma} \varepsilon_{\mathbf{k}} d_{k, \sigma}^{\dagger} d_{k, \sigma}+\sum_{k, \sigma} \varepsilon_{\mathbf{k}+\mathbf{Q}} d_{k+\mathrm{Q}, \sigma}^{\dagger} d_{k+\mathrm{Q}, \sigma} \\
& +\sum_{k, \sigma} D_{k} d_{k, \sigma}^{\dagger} d_{k+\mathrm{Q}, \sigma}+\sum_{k, \sigma} D_{k}^{\dagger} d_{k+\mathrm{Q}, \sigma}^{\dagger} d_{k, \sigma} \\
& +\sum_{k, \sigma} \Delta_{\mathbf{k}}^{\dagger(\mathrm{sc})} d_{-k,-\sigma} d_{k, \sigma}+\sum_{k, \sigma} \Delta_{\mathbf{k}}^{(\mathrm{sc})} d_{k, \sigma}^{\dagger} d_{-k,-\sigma}^{\dagger} \\
& -\mu \sum_{k, \sigma}\left(d_{k, \sigma}^{\dagger} d_{k, \sigma}+d_{k+\mathrm{Q}, \sigma}^{\dagger} d_{k+\mathrm{Q}, \sigma}\right)
\end{aligned}
$$

where $D_{\mathbf{k}}=\left(i \Delta_{\mathbf{k}}\right) \equiv-\sum_{k^{\prime}, \sigma} U\left(\mathbf{k}, \mathbf{k}^{\prime}\right)\left\langle d_{\mathbf{k}^{\prime}+\mathbf{Q}, \sigma}^{\dagger} d_{\mathbf{k}^{\prime}, \sigma}\right\rangle$ and $D_{\mathbf{k}}^{\dagger}=$ $\left(-i \Delta_{\mathbf{k}}\right) \equiv-\sum_{k^{\prime}, \sigma} U\left(\mathbf{k}, \mathbf{k}^{\prime}\right)\left\langle d_{\mathbf{k}^{\prime}, \sigma}^{\dagger} d_{\mathbf{k}^{\prime}+\mathbf{Q}, \sigma}\right\rangle$. The time-reversal invariance of the normal state requires that the dispersion $\varepsilon_{\mathbf{k}}=\varepsilon_{-\mathbf{k}}$. The function $\varepsilon_{\mathbf{k}}$ involves the near neighbor hopping terms to be specified later (see (10)). The gap function $\Delta_{\mathbf{k}}^{\dagger(\mathrm{sc})} \equiv \sum_{k^{\prime}, \sigma} V\left(\mathbf{k}, \mathbf{k}^{\prime}\right)\left\langle d_{k, \sigma}^{\dagger} d_{-k,-\sigma}^{\dagger}\right\rangle=-\Delta_{\mathbf{k}+\mathbf{Q}}^{\dagger}{ }^{(\mathrm{sc})}$. The conical brackets stand for the thermal average calculated with the Hamiltonian in (2). This step ensures the self-consistency.

The imaginary $d$-wave order parameter $D_{\mathbf{k}}$ describing the PG state breaks the time-reversal symmetry of the normal state. The time-reversal operator $\hat{O}$ transforms the order parameter to its complex conjugate: $\hat{O} D_{\mathbf{k}}=D_{\mathbf{k}}^{\dagger}$. If the timereversal symmetry is preserved, $D_{\mathbf{k}}$ and $D_{\mathbf{k}}^{\dagger}$ are identical to within a common spatially independent phase. If, however, the time-reversal symmetry is broken, the two states are distinct albeit with the same free energy. We shall see that the equation corresponding to this gap in the pure DDW case is $D_{\mathbf{k}}^{\dagger} \approx \sum_{\mathbf{k}^{\prime}} U\left(\mathbf{k}, \mathbf{k}^{\prime}\right) D_{\mathbf{k}^{\prime}}^{\dagger} f\left(\mathbf{k}^{\prime}, T\right) \times\left[{\sqrt{\left(\xi_{\mathbf{k}^{\prime}}^{2}+\left|D_{\mathbf{k}^{\prime}}\right|^{2}\right)}}^{-1}\right.$, where the function $f\left(\mathbf{k}^{\prime}, T\right)$ being the difference of two Fermi functions and is positive, the interaction $U(\mathbf{k}, \mathbf{k})$ has to be of the form $U\left(\mathbf{k}, \mathbf{k}^{\prime}\right)=\left|U_{1}\right|\left(\cos k_{x} a-\cos k_{y} a\right)\left(\cos k_{x}^{\prime} a-\cos k_{y}^{\prime} a\right)$. The nonzero DSC order parameter or the gap $\Delta_{k}^{(\text {sc })}$, on the other hand, requires an appropriate attractive interactions $V\left(\mathbf{k}, \mathbf{k}^{\prime}\right)=-\left|V_{1}\right|\left(\cos k_{x} a-\cos k_{y} a\right)\left(\cos k_{x}^{\prime} a-\cos k_{y}^{\prime} a\right)$, where
$V_{1}$ is the coupling strength. The pure DSC gap is given by $\Delta_{\mathbf{k}}^{(\mathrm{sc})}=-(1 / 2) \sum_{\mathbf{k}^{\prime}} V\left(\mathbf{k}, \mathbf{k}^{\prime}\right) \Delta_{\mathbf{k}^{\prime}}^{(\mathrm{sc})} \times\left[{\sqrt{\left(\xi_{\mathbf{k}^{\prime}}^{2}+\Delta_{\mathbf{k}^{\prime}}^{(\mathrm{sc}) 2}\right)}}^{-1}\right.$ which is the usual BCS form. If the pairing interaction $V\left(\mathbf{k}, \mathbf{k}^{\prime}\right)$ is imagined to be a "probe" applied to the Fermi system in the PG state, then the gap function $\Delta_{\mathbf{k}}^{(\mathrm{sc})}$ (where $\Delta_{\mathbf{k}}^{(\mathrm{sc})}=\Delta_{\mathbf{k}}^{\dagger^{(\mathrm{sc})}}$ ) is perhaps a "response" that the system displays. Naturally, the structure of the "probe" in momentum space will have tremendous influence on the "response." For example, the usual electron-phonon (e-ph) type pairing interaction leads to a fully gapped state-a "conventional" BCS superconductor. The electron-bosonic mode (e-bm) interaction or a combination of electron-electron (e-e) and e-bm interactions, on the other hand, are expected to produce gaps with nodes and antinodes (or, more generally, Fermi surface (FS) pockets of the "unconventional" superconductors) and these are interpreted as the manifestation of the non-s-wave symmetry of the order parameter. For a conventional "e-ph pairing interaction" which is structureless in momentum space such a solution of the gap equation would never be possible. Thus, it is natural to surmise, as we have done above, that a combination of e-e and/or e-bm interactions will lead to a $d$-wave gap $\Delta_{k}^{(\text {sc) }}$. For the quantities $\left(\Delta_{\mathbf{k}}, \Delta_{\mathbf{k}}^{(\mathrm{sc})}\right)$, given in the form of $\Delta_{0}(T)\left(\cos k_{x} a-\cos k_{y} a\right)$, the amplitude $\Delta_{0}(T)$ for the two orderings is to be obtained solving a set of self-consistent equations to be specified below.

At this point, we introduce few thermal averages determined by $H$, namely, $G_{\sigma}(\mathbf{k}, \tau)=$ $-\left\langle T\left\{d_{\mathbf{k}, \sigma}(\tau) d_{\mathbf{k}, \sigma}^{\dagger}(0)\right\}\right\rangle, \Gamma_{\sigma}(\mathbf{k}, \tau)=-\left\langle T\left\{d_{-\mathbf{k},-\sigma}^{\dagger}(\tau) d_{\mathbf{k}, \sigma}^{\dagger}(0)\right\}\right\rangle$, $G_{\sigma}^{\prime}(\mathbf{k}, \tau)=-\left\langle T\left\{d_{\mathbf{k}+\mathbf{Q}, \sigma}(\tau) d_{\mathbf{k}, \sigma}^{\dagger}(0)\right\}\right\rangle$, and $\Gamma_{\sigma}^{\prime}(\mathbf{k}, \tau)=$ $-\left\langle T\left\{d_{-\mathbf{k}-\mathbf{Q},-\sigma}^{\dagger}(\tau) d_{\mathbf{k}, \sigma}^{\dagger}(0)\right\}\right\rangle$. Here, $T$ is the time-ordering operator which arranges other operators from right to left in the ascending order of imaginary time $\tau$. The first step of the scheme involves the calculation of (imaginary) time evolution of the operators $d_{\mathbf{k}, \sigma}(\tau)$ where, in units such that $\hbar=1, d_{\mathbf{k}, \sigma}(\tau)=\exp (H \tau) d_{\mathbf{k}, \sigma}(0) \exp (-H \tau)$. We obtain, for example,

$$
\begin{array}{r}
\partial d_{\mathbf{k}, \sigma}=-\left(\varepsilon_{\mathbf{k}}-\mu\right) d_{\mathbf{k}, \sigma}-\Delta_{\mathbf{k}}^{(\mathrm{sc})} d_{-\mathbf{k},-\sigma}^{\dagger}-D_{\mathbf{k}} d_{\mathbf{k}+\mathbf{Q}, \sigma}, \\
\partial d_{-\mathbf{k},-\sigma}^{\dagger}=\left(\varepsilon_{\mathbf{k}}-\mu\right) d_{-\mathbf{k},-\sigma}^{\dagger}-\Delta_{\mathbf{k}}^{\dagger(s c)} d_{\mathbf{k}, \sigma}+D_{\mathbf{k}}^{\dagger} d_{-\mathbf{k}-\mathbf{Q},-\sigma}^{\dagger},
\end{array}
$$

and so on. Here, $\partial \equiv(\partial / \partial \tau)$ and the argument part has been dropped in writing the operators $d_{k, \sigma}(\tau)$ and their derivative. As the next step, upon using (3), we find that the equations of motion of these averages are given by

$$
\begin{aligned}
\partial G_{\sigma}(\mathbf{k}, \tau)= & -\left(\varepsilon_{\mathbf{k}}-\mu\right) G_{\sigma}(\mathbf{k}, \tau)-\Delta_{\mathbf{k}}^{(\mathrm{sc})} \Gamma_{\sigma}(\mathbf{k}, \tau) \\
& -D_{k} G_{\sigma}^{\prime}(\mathbf{k}, \tau)-\delta(\tau), \\
\partial \Gamma_{\sigma}(\mathbf{k}, \tau)= & \left(\varepsilon_{\mathbf{k}}-\mu\right) \Gamma_{\sigma}(\mathbf{k}, \tau)-\Delta_{\mathbf{k}}^{\dagger(s c)} G_{\sigma}(k, \tau) \\
& +D_{\mathbf{k}}^{\dagger} \Gamma_{\sigma}^{\prime}(\mathbf{k}, \tau),
\end{aligned}
$$




$$
\begin{aligned}
\partial G_{\sigma}^{\prime}(\mathbf{k}, \tau)= & -\left(\varepsilon_{\mathbf{k}+\mathbf{Q}}-\mu\right) G_{\sigma}^{\prime}(\mathbf{k}, \tau)-D_{k}^{\dagger} G_{\sigma}(\mathbf{k}, \tau) \\
& -\Delta_{\mathbf{k}+\mathbf{Q}}^{(\mathrm{sc})} \Gamma_{\sigma}^{\prime}(k, \tau), \\
\partial \Gamma_{\sigma}^{\prime}(\mathbf{k}, \tau)= & \left(\varepsilon_{\mathbf{k}+\mathbf{Q}}-\mu\right) \Gamma_{\sigma}^{\prime}(\mathbf{k}, \tau)+D_{\mathbf{k}} \Gamma_{\sigma}(\mathbf{k}, \tau) \\
& -\Delta_{\mathbf{k}+\mathbf{Q}}^{\dagger}{ }^{(s c)} G_{\sigma}^{\prime}(\mathbf{k}, \tau) .
\end{aligned}
$$

The final step is the calculation of the Fourier coefficients: $G_{\sigma}^{\lambda}\left(\mathbf{k}, \omega_{n}\right)=\int_{0}^{\beta} d \tau e^{i \omega n \tau} G_{\sigma}^{\lambda}(\mathbf{k}, \tau)$ (where the Matsubara frequencies are $\omega_{n}=[(2 n+1) \pi / \beta]$ with $\left.n=0, \pm 1, \pm 2, \ldots\right)$ of these temperature Green's functions. Here, $\beta=\left(k_{B} T\right)^{-1}$. We refrain from writing explicitly the equations to determine these coefficients as this is a trivial exercise in view of (4). Upon solving the equations, we obtain $G_{\sigma}\left(\mathbf{k}, \omega_{n}\right)=\left(\Delta_{1} / \Delta\right)$, $\Gamma_{\sigma}\left(\mathbf{k}, \omega_{n}\right)=\left(\Delta_{2} / \Delta\right), G_{\sigma}^{\prime}\left(\mathbf{k}, \omega_{n}\right)=\left(\Delta_{3} / \Delta\right)$, and $\Gamma_{\sigma}^{\prime}\left(\mathbf{k}, \omega_{n}\right)=$ $\left(\Delta_{4} / \Delta\right)$, where

$$
\begin{aligned}
\Delta_{1}= & \left(i \omega_{n}+\xi_{\mathbf{k}}\right)\left(\left(i \omega_{n}\right)^{2}-\xi_{\mathbf{k}+\mathbf{Q}}^{2}-\Delta_{\mathbf{k}}^{(\mathrm{sc}) 2}\right) \\
& -\Delta_{\mathbf{k}}^{2}\left(i \omega_{n}-\xi_{\mathbf{k}+\mathbf{Q}}\right), \Delta_{2} \\
= & \left(\left(i \omega_{n}\right)^{2}-\xi_{\mathbf{k}+\mathbf{Q}}^{2}-D_{\mathrm{eff}}(\mathbf{k})^{2}\right) \Delta_{\mathbf{k}}^{(\mathrm{sc})}, \Delta_{3} \\
= & D_{k}^{\dagger}\left[\left(i \omega_{n}+\xi_{k}\right)\left(i \omega_{n}+\xi_{k+\mathbf{Q}}\right)-D_{\mathrm{eff}}(\mathbf{k})^{2}\right], \Delta_{4} \\
= & D_{k}^{\dagger} \Delta_{\mathbf{k}}^{(\mathrm{sc})}\left(\xi_{\mathbf{k}}+\xi_{\mathbf{k}+\mathbf{Q}}\right), \Delta \\
= & \left(\left(i \omega_{n}\right)^{2}-\xi_{\mathbf{k}}^{2}\right)\left(\left(i \omega_{n}\right)^{2}-\xi_{\mathbf{k}+\mathbf{Q}}^{2}\right) \\
& -\left\{\left(i \omega_{n}+\xi_{\mathbf{k}}\right)\left(i \omega_{n}+\xi_{\mathbf{k}+\mathbf{Q}}\right)\right. \\
& \left.+\left(i \omega_{n}-\xi_{\mathbf{k}}\right)\left(i \omega_{n}-\xi_{\mathbf{k}+\mathbf{Q}}\right)\right\} \Delta_{\mathbf{k}}^{2} \\
& -\Delta_{\mathbf{k}}^{(\mathrm{sc}) 2}\left(\left(i \omega_{n}\right)^{2}-\xi_{\mathbf{k}}^{2}+\left(i \omega_{n}\right)^{2}-\xi_{\mathbf{k}+\mathbf{Q}}^{2}\right) \\
& +\left(\Delta_{\mathbf{k}}^{(\mathrm{scc}) 4}+\Delta_{\mathbf{k}}^{4}+2 \Delta_{\mathbf{k}}^{2} \Delta_{\mathbf{k}}^{(\mathrm{sc}) 2}\right),
\end{aligned}
$$

$\xi_{\mathbf{k}}=\varepsilon_{\mathbf{k}}-\mu$, and $D_{\text {eff }}^{2}(\mathbf{k})=\left(\Delta_{\mathbf{k}}^{2}+\Delta_{\mathbf{k}}^{(\mathrm{sc}) 2}\right)$. The denominator $\Delta$ of the Fourier coefficients yields the single particle excitation spectrum. We find that this is a quadratic in $\left(i \omega_{n}\right)^{2}$ :

$$
\begin{aligned}
\Delta= & \left(i \omega_{n}\right)^{4}-\left(i \omega_{n}\right)^{2}\left(\xi_{\mathbf{k}}^{2}+\xi_{\mathbf{k}+\mathbf{Q}}^{2}+2 D_{\text {eff }}(\mathbf{k})^{2}\right) \\
+ & \left\{\xi_{\mathbf{k}}^{2} \xi_{\mathbf{k}+\mathbf{Q}}^{2}-2 \xi_{\mathbf{k}} \xi_{\mathbf{k}+\mathbf{Q}} \Delta_{\mathbf{k}}^{2}+\Delta_{\mathbf{k}}^{(\mathrm{sc}) 2}\left(\xi_{\mathbf{k}}^{2}+\xi_{\mathbf{k}+\mathbf{Q}}^{2}\right)\right. \\
+ & \left.\left(\Delta_{\mathbf{k}}^{(\mathrm{sc}) 4}+\Delta_{\mathbf{k}}^{4}+2 \Delta_{\mathbf{k}}^{2} \Delta_{\mathbf{k}}^{(\mathrm{sc}) 2}\right)\right\} .
\end{aligned}
$$

It may be noted that, in the pure $d$-wave case to be investigated below, $\left|D_{\mathbf{k}}\right|^{2}$ will get replaced by $\Delta_{\mathbf{k}}^{2}$. Correspondingly, the single-particle excitation spectra will be given by $E_{\mathbf{k}}(T<$ $\left.T_{c}\right)= \pm E^{(U, L)}(\mathbf{k}), E_{\mathbf{k}}^{(U, L) 2}=(1 / 2)\left[\left(\xi_{\mathbf{k}}^{2}+\xi_{\mathbf{k}+\mathbf{Q}}^{2}+2 D_{\text {eff }}(\mathbf{k})^{2}\right) \pm\right.$ $p(\mathbf{k})]$, and $p(\mathbf{k})=\left[\left(\xi_{\mathbf{k}}^{2}-\xi_{\mathbf{k}+\mathbf{Q}}^{2}\right)^{2}+4 \Delta_{\mathbf{k}}^{2}\left(\xi_{\mathbf{k}}+\xi_{\mathbf{k}+\mathbf{Q}}\right)^{2}\right]^{1 / 2}$. The quasiparticle excitations in cuprates (where we have a pure $d$-wave SC order together with a coexistent pseudogap right up to $0 \mathrm{~K}$ ) are thus demonstrably Bogoliubov quasiparticles in the SC phase. It may be noted that the result $E_{\mathbf{k}}(T<$ $\left.T_{c}\right)= \pm E^{(U, L)}(\mathbf{k})$ obtained here is different from the one surmised by Leblanc et al. [41], within the ansatz for the RVB state proposed by Yang et al. [42, 43], to explain the angle-resolved photoemission spectroscopy (ARPES) data published by Hashimoto and his collaborators [24]. The conjectured energy of the gapped excitations in the superconducting state is $E_{\mathrm{sc}}^{\sigma}=\sqrt{\left\{\left(E^{\sigma}\right)^{2}+\Delta_{\mathrm{sc}}^{2}\right\}}$, with Bogoliubov amplitudes $u_{\sigma}^{2}=\left(1+E^{\sigma} / E_{\mathrm{sc}}^{\sigma}\right) / 2$ and $v_{\sigma}^{2}=\left(1-E^{\sigma} / E_{\mathrm{sc}}^{\sigma}\right) / 2$ which are applied to the pseudogapped bands indexed by $\sigma= \pm$ and given as $E^{ \pm}=\varepsilon_{1} \pm \sqrt{\left\{\varepsilon_{2}^{2}+\Delta_{\mathrm{PG}}^{2}\right\}}$. The energies $\varepsilon_{1}=\left(\epsilon_{\mathbf{k}}-\epsilon_{\mathbf{k}}^{0}\right) / 2$ and $\varepsilon_{2}=\left(\epsilon_{\mathbf{k}}+\epsilon_{\mathbf{k}}^{0}\right) / 2$, where $\epsilon_{\mathbf{k}}$ is a third nearest neighbor tightbinding dispersion and $\epsilon_{\mathbf{k}}^{0}$ is that for first nearest neighbor which for $\epsilon_{\mathbf{k}}^{0}=0$ defines the antiferromagnetic Brillouin zone boundary. Obviously enough, the difference between our result and that proposed by LeBlanc et al. [41] lies in the nonmerger of the two gaps $\Delta_{\mathbf{k}}$ and $\Delta_{\mathbf{k}}^{(\mathrm{sc})}$ in the excitation spectrum of hole-doped cuprates into one "quadrature gap" in the former. Despite this, as we shall see below, we could capture qualitatively some key aspects (see Figure 4) of the results obtained by Hashimoto and his coworkers [24].

At this point, we note that many theorists and experimentalists $[22,23,28-30]$ subscribe to the view that the pseudogap is of $d+i d$ variety (chiral DDW (CDDW) order at the wave vector $\mathbf{Q}=( \pm \pi, \pm \pi))$. In such a situation, $D_{\mathbf{k}}=\left(-\chi_{\mathbf{k}}+i \Delta_{\mathbf{k}}\right)$, where the real and the imaginary parts $\left(\chi_{\mathbf{k}}, \Delta_{\mathbf{k}}\right)$ are given by $\chi_{\mathbf{k}}=-\chi_{0} \sin \left(k_{x} a\right) \sin \left(k_{y} a\right)$, and $\Delta_{\mathbf{k}}=\left(\Delta_{0}^{(\mathrm{PG})}(T)\right)\left(\cos k_{x} a-\cos k_{y} a\right)$. The quantities $\left(\Delta, \Delta_{1}, \Delta_{2}, \Delta_{3}, \Delta_{4}\right)$ in (5) would now be given by

$$
\begin{aligned}
\Delta= & \left(i \omega_{n}\right)^{4}-\left(i \omega_{n}\right)^{2}\left(\xi_{\mathbf{k}}^{2}+\xi_{\mathbf{k}+\mathbf{Q}}^{2}+2 D_{\mathrm{eff}}(\mathbf{k})^{2}\right) \\
& +\left\{\xi_{\mathbf{k}}^{2} \xi_{\mathbf{k}+\mathbf{Q}}^{2}-2 \xi_{\mathbf{k}} \xi_{\mathbf{k}+\mathbf{Q}}\left|D_{\mathbf{k}}\right|^{2}+\Delta_{\mathbf{k}}^{(\mathrm{sc}) 2}\left(\xi_{\mathbf{k}}^{2}+\xi_{\mathbf{k}+\mathbf{Q}}^{2}\right)\right. \\
& \left.+\Delta_{\mathbf{k}}^{(\mathrm{sc}) 4}+\left|D_{\mathbf{k}}\right|^{4}-\left(D_{\mathbf{k}}^{\dagger}\right)^{2} \Delta_{\mathbf{k}}^{(\mathrm{sc} 2}-\left(D_{\mathbf{k}}\right)^{2} \Delta_{\mathbf{k}}^{(\mathrm{sc}) 2}\right\}, \Delta_{1} \\
= & \left(i \omega_{n}+\xi_{\mathbf{k}}\right)\left(\left(i \omega_{n}\right)^{2}-\xi_{\mathbf{k}+\mathbf{Q}}^{2}-\Delta_{\mathbf{k}}^{(\mathrm{sc}) 2}\right) \\
& -\left|D_{\mathbf{k}}\right|^{2}\left(i \omega_{n}-\xi_{\mathbf{k}+\mathbf{Q}}\right), \Delta_{2} \\
= & \Delta_{\mathbf{k}}^{(\mathrm{sc})}\left\{\left(i \omega_{n}\right)^{2}-\xi_{\mathbf{k}+\mathbf{Q}}^{2}-\Delta_{\mathbf{k}}^{(\mathrm{sc}) 2}+\left(D_{\mathbf{k}}^{\dagger}\right)^{2}\right\}, \Delta_{3} \\
= & D_{\mathbf{k}}^{\dagger}\left(i \omega_{n}+\xi_{\mathbf{k}}\right)\left(i \omega_{n}+\xi_{\mathbf{k}+\mathbf{Q}}\right)-D_{\mathbf{k}}\left(-\Delta_{\mathbf{k}}^{(\mathrm{scc}) 2}+\left(D_{\mathbf{k}}^{\dagger}\right)^{2}\right), \Delta_{4} \\
= & D_{\mathbf{k}}^{\dagger} \Delta_{\mathbf{k}}^{(\mathrm{scc})}\left(i \omega_{n}+\xi_{\mathbf{k}}\right)+D_{\mathbf{k}} \Delta_{\mathbf{k}}^{(\mathrm{sc})}\left(i \omega_{n}-\xi_{\mathbf{k}+\mathbf{Q}}\right),
\end{aligned}
$$

where $D_{\text {eff }}(\mathbf{k})^{2}$ here is equal to $\left(\left|D_{\mathbf{k}}\right|^{2}+\Delta_{\mathbf{k}}^{(\mathrm{sc}) 2}\right)$. The single particle excitation spectrum $E_{\mathbf{k}}\left(T<T_{c}\right)$ is given by $E_{\mathbf{k}}\left(T<T_{c}\right)= \pm E^{(U, L)}(\mathbf{k}), E_{\mathbf{k}}^{(U, L) 2}=$ $(1 / 2)\left[\left(\xi_{\mathbf{k}}^{2}+\xi_{\mathbf{k}+\mathbf{Q}}^{2}+2 D_{\text {eff }}(\mathbf{k})^{2}\right) \pm p(\mathbf{k})\right]$, and $p(\mathbf{k})=\left[\left(\xi_{\mathbf{k}}^{2}-\xi_{\mathbf{k}+\mathbf{Q}}^{2}\right)^{2}+\right.$ $\left.4\left|D_{\mathbf{k}}\right|^{2}\left(\xi_{\mathbf{k}}+\xi_{\mathbf{k}+\mathbf{Q}}\right)^{2}+4\left\{\left(D_{\mathbf{k}}^{\dagger}\right)^{2}+\left(D_{\mathbf{k}}\right)^{2}-2\left|D_{\mathbf{k}}\right|^{2}\right\} \Delta_{\mathbf{k}}^{(\mathrm{sc}) 2}\right]^{1 / 2}$. Unlike the pure DDW case, for the CDDW case 
$p(\mathbf{k})=4 i \Delta_{\mathbf{k}} \Delta_{\mathbf{k}}^{(\mathrm{sc})}$ when the dispersion is perfectly nested. Thus, $E_{\mathbf{k}}^{(U, L)}= \pm\left[\xi_{\mathbf{k}}^{2}+D_{\text {eff }}(\mathbf{k})^{2} \pm 2 i \Delta_{\mathbf{k}} \Delta_{\mathbf{k}}^{(\mathrm{sc})}\right]^{1 / 2}$ and the Fourier coefficient $G_{\sigma}\left(\mathbf{k}, \omega_{n}\right)=\sum_{j= \pm 1}\left\{U_{\mathbf{k}}^{(j)^{2}}\left(i \omega_{n}+j\left(\dot{\varepsilon}_{\mathbf{k}}+i_{\mathbf{k}}^{-1}\right)\right)^{-1}+\right.$ $\left.V_{\mathbf{k}}^{(j)^{2}}\left(i \omega_{n}+j\left(\dot{\varepsilon}_{\mathbf{k}}-i \tau_{\mathbf{k}}^{-1}\right)\right)^{-1}\right\}$, where $\dot{\varepsilon}_{\mathbf{k}}=R_{\mathbf{k}}^{1 / 2} \cos \left(\theta_{\mathbf{k}} / 2\right)$, $i_{\mathbf{k}}^{-1}=R_{\mathbf{k}}^{1 / 2} \sin \left(\theta_{\mathbf{k}} / 2\right), R_{\mathbf{k}}=\left[\left(\xi_{\mathbf{k}}^{2}+D_{\mathrm{eff}}(\mathbf{k})^{2}\right)^{2}+4 \Delta_{\mathbf{k}}^{2} \Delta_{\mathbf{k}}^{(\mathrm{sc}) 2}\right]^{1 / 2}$, $\tan ^{2}\left(\theta_{\mathbf{k}}\right)=\left(4 \Delta_{\mathbf{k}}^{2} \Delta_{\mathbf{k}}^{(\mathrm{sc}) 2}\right) /\left(\xi_{\mathbf{k}}^{2}+D_{\text {eff }}(\mathbf{k})^{2}\right)^{2}, U_{\mathbf{k}}^{(j)^{2}}=$ $(1 / 4)\left[1-\left(j \xi_{\mathbf{k}} / R_{\mathbf{k}}^{1 / 2} \exp \left(i \theta_{\mathbf{k}} / 2\right)\right)\right]$, and $V_{\mathbf{k}}^{(j)^{2}}=(1 / 4)[1-$ $\left.\left(j \xi_{\mathbf{k}} / R_{\mathbf{k}}^{1 / 2} \exp \left(i \theta_{\mathbf{k}} / 2\right)\right)\right]$. The sum of the coherence factors $\sum_{j= \pm 1}\left\{U_{\mathbf{k}}^{(j)^{2}}+V_{\mathbf{k}}^{(j)^{2}}\right\}=1$, but the factors are complex unlike the usual Bogoluibov picture. The outcome suggests that the investigation on the possibility of CDDW + DSC state requires a deeper analysis. We shall, therefore, presently focus our attention on the imperfect nesting and the pure DDW scenario.

It may be seen that, in the pure DDW case, $\Delta=\left\{\left(i \omega_{n}+\right.\right.$ $\left.\left.\xi_{\mathbf{k}}\right)\left(i \omega_{n}+\xi_{\mathbf{k}+\mathbf{Q}}\right)-\Delta_{\mathbf{k}}^{2}\right\} \times\left\{\left(i \omega_{n}-\xi_{\mathbf{k}}\right)\left(i \omega_{n}-\xi_{\mathbf{k}+\mathbf{Q}}\right)-\Delta_{\mathbf{k}}^{2}\right\}$ and

$$
\begin{aligned}
& G_{\sigma}\left(\mathbf{k}, \omega_{n}\right)=\frac{\left(i \omega_{n}-\xi_{\mathbf{k}+\mathbf{Q}}\right)}{\left\{\left(i \omega_{n}-\xi_{\mathbf{k}}\right)\left(i \omega_{n}-\xi_{\mathbf{k}+\mathbf{Q}}\right)-\Delta_{\mathbf{k}}^{2}\right\}}, \\
& G_{\sigma}^{\prime}\left(\mathbf{k}, \omega_{n}\right)=\frac{D_{\mathbf{k}}^{\dagger}}{\left\{\left(i \omega_{n}-\xi_{\mathbf{k}}\right)\left(i \omega_{n}-\xi_{\mathbf{k}+\mathbf{Q}}\right)-\Delta_{\mathbf{k}}^{2}\right\}},
\end{aligned}
$$

which may be written as

$$
\begin{gathered}
G_{\sigma}\left(\mathbf{k}, \omega_{n}\right)=\left\{u_{\mathbf{k}}^{2}\left(i \omega_{n}-\varepsilon_{k}^{(U)}\right)^{-1}+v_{\mathbf{k}}^{2}\left(i \omega_{n}-\varepsilon_{k}^{(L)}\right)^{-1}\right\}, \\
G_{\sigma}^{\prime}\left(\mathbf{k}, \omega_{n}\right)=\left(\frac{D_{\mathbf{k}}^{\dagger}}{2 w_{k}}\right)\left\{\left(i \omega_{n}-\varepsilon_{k}^{(U)}\right)^{-1}-\left(i \omega_{n}-\varepsilon_{k}^{(L)}\right)^{-1}\right\} .
\end{gathered}
$$

The first result in (9), upon applying Luttinger's theorem [44], leads to the equation to determine the chemical potential $\mu:(1+p)=\left(N_{s} / 2\right)^{-1} \sum_{\mathbf{k}^{\prime}}\left[u_{\mathbf{k}^{\prime}}^{2} \times\right.$ $\left.\left(\exp \left(\beta \varepsilon_{\mathbf{k}^{\prime}}^{(U)}\right)+1\right)^{-1}+v_{\mathbf{k}^{\prime}}^{2} \times\left(\exp \left(\beta \varepsilon_{\mathbf{k}^{\prime}}^{(L)}\right)+1\right)^{-1}\right]$, where $p$ is the hole-doping level, and $N_{s}$ is the number of unit cells in the $\mathbf{k}$-space. The second result in (9) leads to the DDW gap equation $D_{\mathbf{k}}^{\dagger}=\sum_{\mathbf{k}^{\prime}, \sigma} U\left(\mathbf{k}, \mathbf{k}^{\prime}\right)\left(D_{\mathbf{k}^{\prime}}^{\dagger} / 2 w_{\mathbf{k}^{\prime}}\right)\left[\left(\exp \left(\beta \varepsilon_{\mathbf{k}^{\prime}}^{(L)}\right)+1\right)^{-1}-\right.$ $\left.\left(\exp \left(\beta \varepsilon_{\mathbf{k}^{\prime}}^{(U)}\right)+1\right)^{-1}\right]$. Quite obviously, the difference of the Fermi functions within the square brackets is positive and therefore the interaction $U\left(\mathbf{k}, \mathbf{k}^{\prime}\right)$ needs to be repulsive for this equation to be meaningful. The quasiparticle coherence factors $\left(u_{\mathbf{k}}^{2}, v_{\mathbf{k}}^{2}\right)$ are given by the expressions $u_{\mathbf{k}}^{2}=(1 / 2)[1+$ $\left.\left(\varepsilon_{\mathbf{k}}^{L} / w_{\mathbf{k}}\right)\right], v_{\mathbf{k}}^{2}=(1 / 2)\left[1-\left(\varepsilon_{\mathbf{k}}^{L} / w_{\mathbf{k}}\right)\right]$. Here $\varepsilon_{\mathbf{k}}^{(U, L)}=\left[\varepsilon_{\mathbf{k}}^{U}+\right.$ $\left.j w_{\mathbf{k}}\right], \varepsilon_{\mathbf{k}}^{U}=\left(\xi_{\mathbf{k}}+\xi_{\mathbf{k}+\mathbf{Q}}\right) / 2, \varepsilon_{\mathbf{k}}^{L}=\left(\xi_{\mathbf{k}}-\xi_{\mathbf{k}+\mathbf{Q}}\right) / 2$, and $w_{\mathbf{k}}=$ $\left[\left(\varepsilon_{\mathbf{k}}^{L}\right)^{2}+\left|D_{\mathbf{k}}\right|^{2}\right]^{1 / 2}$. The index $j$ is equal to $( \pm 1)$ with $j=$ +1 corresponding to the upper branch $(U)$ and $j=-1$ corresponding to the lower branch $(L)$. The single-particle spectral function in the spin- $\sigma$ channel is given by $A_{\sigma}(\mathbf{k}, \omega)=$ $\left(-\pi^{-1}\right) \operatorname{Im} G_{\sigma}^{(R)}(\mathbf{k}, \omega)$, where $G_{\sigma}^{(R)}(\mathbf{k}, \omega)$ is the retarded Green's function given by $G_{\sigma}^{R}(k, \omega)=\int_{-\infty}^{\infty}\left(d \omega^{\prime} / 2 \pi\right)\left\{\zeta_{\sigma}\left(k, \omega^{\prime}\right) /(\omega-\right.$ $\left.\left.\omega^{\prime}+i 0^{+}\right)\right\}$and $\zeta_{\sigma}(k, \omega)=(1 / i)\left\{\left.G_{\sigma}\left(k, z=i \omega_{n}\right)\right|_{z=\omega-i 0^{+}}-\right.$ $\left.\left.G_{\sigma}\left(k, z=i \omega_{n}\right)\right|_{z=\omega+i 0^{+}}\right\}$. Upon using the result $\left(x \pm i 0^{+}\right)^{-1}=$ $\left[P\left(x^{-1}\right) \pm(1 / i) \pi \delta(x)\right]$, where $P$ represents a Cauchy's principal value, the spectral function $A(\mathbf{k}, \omega)$ in the DDW phase is given by a sum of $\delta$ functions at the quasiparticle energies: $A(\mathbf{k}, \omega)=2 \pi\left[u_{\mathbf{k}}^{2} \delta\left(\omega-\varepsilon_{\mathbf{k}}^{(U)}\right)+v_{\mathbf{k}}^{2} \delta\left(\omega-\varepsilon_{\mathbf{k}}^{(L)}\right)\right]$. These results are the same as those reported by Chakravarty et al. [9-11]. In particular, if the dispersion is nested, we obtain Bogoluibov-like dispersion $\varepsilon_{\mathbf{k}}^{(U, L)}=j w_{\mathbf{k}}$, and $D_{\mathbf{k}}^{\dagger}=$ $\sum_{\mathbf{k}^{\prime}, \sigma} U\left(\mathbf{k}, \mathbf{k}^{\prime}\right)\left(D_{\mathbf{k}^{\prime}}^{\dagger} / 2 w_{\mathbf{k}^{\prime}}\right) \tanh \left(\beta w_{\mathbf{k}^{\prime}} / 2\right)$, where $\beta=\left(k_{B} T\right)^{-1}$, but the coherence factors $u_{\mathbf{k}}^{2}=1$ and $v_{\mathbf{k}}^{2}=0$. This situation being inadmissible as it effectively corresponds to a single semimetallic band, we need to specify the normal-state dispersion at this stage. It is well known [45] that near a van Hove singularity (vHS) the fermion density of states diverges, so that even arbitrarily weak interactions can produce large effects. When the Fermi level reaches these points, a variety of response functions diverge. As already stated, we have a twodimensional fermionic system with a square lattice. Suppose we have a tight-binding dispersion of the form

$$
\begin{aligned}
\varepsilon_{\mathbf{k}}= & -2 t_{1}\left(\cos \left(k_{x} a\right)+\cos \left(k_{y} a\right)\right)+4 t_{2} \cos \left(k_{x} a\right) \\
& \times \cos \left(k_{y} a\right)-2 t_{3}\left(\cos 2 k_{x} a+\cos 2 k_{y} a\right)+4 t_{2},
\end{aligned}
$$

where, for the hole-doped materials, $t_{2}>0$ (for the electrondoped materials $\left.t_{2}<0\right)$, and, in all cases, $t_{2}<\left(t_{1} / 2\right)$. For example, typical values are $t_{1} \sim 0.2 \mathrm{eV},\left(t_{2} / t_{1}\right) \sim 0.24$, and $\left(t_{3} / t_{1}\right) \sim 0.01$. Upon ignoring the third neighbor hopping term above, we find that the dispersion typically has two inequivalent saddle points at $(\pi, 0)$ and $(0, \pi)$ in the first Brillouin zone. Upon assuming that for fillings such that the Fermi curve lies close to the singularities, the majority of states participating in the pairing formation will come from regions in the vicinity of these saddle points. This is the key strategy we adopt below to plot the single-particle excitation spectrum and calculate all quantities of interest, such as the the thermodynamic and transport properties. As we shall show below, the anomalous temperature dependence of the electronic specific heat (a typical non-Fermi liquid (NFL) behavior) due to the onset of exotic DDW ordering has its origin in the nonnesting property of the dispersion in (10).

The k-summation in the equations for $D_{\mathbf{k}}^{\dagger}$ and $\mu$ in the preceeding paragraph may be replaced by the integration: $\int d(\mathbf{k} a) \rho_{\text {Fermi }}(\mathbf{k}) \rightarrow$ $\int_{-\pi}^{+\pi} d\left(k_{x} a\right) / 2 \pi \int_{-\pi}^{+\pi}\left(d\left(k_{y} a\right) / 2 \pi \rho_{\text {Fermi }}(\mathbf{k})\right.$, where the Fermi energy density of states (DOSs) $\rho_{\text {Fermi }}(\mathbf{k})$ should be determined by the inclusion of the disorder potential ideally (see Section 3.2). The quantity $\rho_{\text {Fermi }}(\mathbf{k})$ here is obtained from the spectral function $A(\mathbf{k}, \omega)$ given below (9) by a sum of $\delta$ functions at the quasiparticle energies. We simply replace the $\delta$ functions by Lorentzians with an assumed intrinsic lifetime broadening $\left(\gamma / t_{1}\right) \sim 0.1$. A 2D plot of $A(\omega)=$ $\int d(\mathbf{k} a) A(\mathbf{k}, \omega)=\int_{-\pi}^{+\pi} d\left(k_{x} a\right) / 2 \pi \int_{-\pi}^{+\pi} d\left(k_{y} a\right) / 2 \pi A(\mathbf{k}, \omega)$ as a function of $\omega$, in the pseudogap phase (doping level 9.94\%), is shown in Figure 2. The numerical values, in the units of 
the first neighbor hopping $t_{1}$, are $\left(\mu / t_{1}\right)=-0.0189$, the PG gap amplitude $\left(\Delta_{0}^{(\mathrm{pg})} / t_{1}\right)=0.0871$, and the SC gap amplitude $\left(\Delta_{0}^{(\mathrm{sc})} / t_{1}\right)=0$. The hopping parameters are $\left(t_{2} / t_{1}\right)=0.3925$, and $\left(t_{3} / t_{1}\right)=0.0005$. The DDW ordering leads to pining of the Fermi level close to, but not precisely at, the vHs. The plot shows a cusp at $\omega=0$. For comparison purpose, we have plotted the square lattice tight band DOS in the Hubbard model as well which clearly shows vHS. There is logarithmic singularity at the centre (saddle point singularity) and the step-like discontinuities at the band-edges [46].

In the absence of the DDW gap, with the modulation vector $\mathbf{Q}$ set at $(0,0)$ and $\left|D_{\mathbf{k}}\right|=0$, we have $\Delta=$ $\left(\left(i \omega_{n}\right)^{2}-\hat{\varepsilon}^{2}(\mathbf{k})\right)\left(\left(i \omega_{n}\right)^{2}-\xi_{\mathbf{k}}^{2}\right), \Delta_{1}=\left(i \omega_{n}+\xi_{\mathbf{k}}\right)\left(\left(i \omega_{n}\right)^{2}-\right.$ $\left.\xi_{\mathbf{k}}^{2}\right)$, and $\Delta_{2}=\left(\left(i \omega_{n}\right)^{2}-\xi_{\mathbf{k}}^{2}\right) \Delta_{\mathbf{k}}^{(\mathrm{sc})}$. This yields $G_{\sigma}\left(\mathbf{k}, \omega_{n}\right)=$ $a_{\mathbf{k}}^{(-)}\left(i \omega_{n}+\dot{\varepsilon}(\mathbf{k})\right)^{-1}+a_{\mathbf{k}}^{(+)}\left(i \omega_{n}-\dot{\varepsilon}(\mathbf{k})\right)^{-1}$, and $\Gamma_{\sigma}\left(\mathbf{k}, \omega_{n}\right)=$ $-\left(\Delta_{\mathbf{k}}^{(\mathrm{sc})} / 2 \dot{\varepsilon}(\mathbf{k})\right)\left[\left(i \omega_{n}+\dot{\varepsilon}(\mathbf{k})\right)^{-1}-\left(i \omega_{n}-\dot{\varepsilon}(\mathbf{k})\right)^{-1}\right]$, where $\dot{\varepsilon}(\mathbf{k})=$ $\sqrt{\left(\xi_{\mathbf{k}}^{2}+\left|\Delta_{\mathbf{k}}^{(\mathrm{sc})}\right|^{2}\right)}$ and the coherence factors are given by $a_{\mathbf{k}}^{( \pm)}=(1 / 2)\left[1 \pm\left(\xi_{\mathbf{k}} / \hat{\varepsilon}(\mathbf{k})\right)\right]$. Upon using the expression for the Fourier coefficient $G_{\sigma}\left(\mathbf{k}, \omega_{n}\right)$ the chemical potential $\mu$, according to the Luttinger rule [34], is given by the equation $(1+p)=\left(N_{s} / 2\right)^{-1} \sum_{k}\left[\left(1-\left(\xi_{\mathbf{k}} / \hat{\varepsilon}(\mathbf{k})\right) \times(\exp (-\beta \dot{\varepsilon}(\mathbf{k}))+1)^{-1}+\right.\right.$ $\left(1+\left(\xi_{\mathbf{k}} / \mathcal{\varepsilon}^{\prime}\left(\mathbf{k}^{\prime}\right)\right) \times\left(\exp \left(\beta \dot{\varepsilon}^{\prime}\left(\mathbf{k}^{\prime}\right)\right)+1\right)^{-1}\right]$, where $p$ is the doping level and $N_{s}$ is the number of $\mathbf{k}$-vectors in the first Brillouin zone. The Fourier coefficient $\Gamma_{\sigma}\left(\mathbf{k}, \omega_{n}\right)$ leads to the weak coupling BCS gap equation for the singlet pairing: $\Delta_{\mathbf{k}}^{(\mathrm{sc})}=-\sum_{\mathbf{k}^{\prime}} V\left(\mathbf{k}, \mathbf{k}^{\prime}\right)\left(\Delta_{\mathbf{k}^{\prime}}^{(\mathrm{sc})} / 2 \dot{\varepsilon}\left(\mathbf{k}^{\prime}\right)\right) \tanh \left(\beta \dot{\varepsilon}^{\prime}\left(\mathbf{k}^{\prime}\right) / 2\right)$, where $\beta=\left(k_{B} T\right)^{-1}$. We, thus, notice that the Matsubara propagators obtained in our general analysis, where the DDW and DSC orderings have been assumed to be coexisting, are able to yield the already known results [9-11] albeit with slightly different expression for the excitation spectrum.

For the coexistent DDW and DSC state, from (5) and (6) we find that

$$
\begin{aligned}
& G_{\sigma}\left(\mathbf{k}, \omega_{n}\right)=\left\{u_{\mathbf{k}}^{(+)^{2}}\left(i \omega_{n}+E_{\mathbf{k}}^{(U)}\right)^{-1}+u_{\mathbf{k}}^{(-)^{2}}\left(i \omega_{n}-E_{\mathbf{k}}^{(U)}\right)^{-1}\right.\left.+v_{\mathbf{k}}^{(+)^{2}}\left(i \omega_{n}+E_{\mathbf{k}}^{(L)}\right)^{-1}+v_{\mathbf{k}}^{(-)^{2}}\left(i \omega_{n}-E_{\mathbf{k}}^{(L)}\right)^{-1}\right\}, \\
& u_{\mathbf{k}}^{(+)^{2}}=\left(\frac{1}{2}\right)\left[\left(1-\left(\frac{\xi_{\mathbf{k}}}{E_{\mathbf{k}}^{(U)}}\right)\right) \times\left\{\frac{\left(E_{\mathbf{k}}^{(U) 2}-\xi_{\mathbf{k}+\mathbf{Q}}^{\prime}\right)}{q(\mathbf{k})}\right\}\right. \\
&\left.-\left\{\left(1+\left(\frac{\xi_{\mathbf{k}+\mathbf{Q}}}{E_{\mathbf{k}}^{(U)}}\right)\right) \times\left(\frac{\Delta_{\mathbf{k}}^{2}}{q(\mathbf{k})}\right)\right\}\right], \\
& u_{\mathbf{k}}^{(-)^{2}}=\left(\frac{1}{2}\right)\left[\left(1+\left(\frac{\xi_{\mathbf{k}}}{E_{\mathbf{k}}^{(U)}}\right)\right) \times\left\{\frac{\left(E_{\mathbf{k}}^{(U) 2}-\xi_{\mathbf{k}+\mathbf{Q}}^{\prime}\right)}{q(\mathbf{k})}\right\}\right. \\
&\left.-\left\{\left(1-\left(\frac{\xi_{\mathbf{k}+\mathbf{Q}}}{E_{\mathbf{k}}^{(U)}}\right)\right) \times\left(\frac{\Delta_{\mathbf{k}}^{2}}{q(\mathbf{k})}\right)\right\}\right],
\end{aligned}
$$

$$
\begin{aligned}
& v_{\mathbf{k}}^{(+)^{2}}=\left(-\frac{1}{2}\right) {\left[\left(1-\left(\frac{\xi_{\mathbf{k}}}{E_{\mathbf{k}}^{(L)}}\right)\right) \times\left\{\frac{\left(E_{\mathbf{k}}^{(L) 2}-\xi_{\mathbf{k}+\mathbf{Q}}^{\prime}\right)}{q(\mathbf{k})}\right\}\right.} \\
&\left.-\left\{\left(1+\left(\frac{\xi_{\mathbf{k}+\mathbf{Q}}}{E_{\mathbf{k}}^{(L)}}\right)\right) \times\left(\frac{\Delta_{\mathbf{k}}^{2}}{q(\mathbf{k})}\right)\right\}\right], \\
& v_{\mathbf{k}}^{(-)^{2}}=\left(-\frac{1}{2}\right)\left[\left(1+\left(\frac{\xi_{\mathbf{k}}}{E_{\mathbf{k}}^{(L)}}\right)\right) \times\left\{\frac{\left(E_{\mathbf{k}}^{(L) 2}-\xi_{\mathbf{k}+\mathbf{Q}}^{\prime}\right)}{q(\mathbf{k})}\right\}\right. \\
&\left.-\left\{\left(1-\left(\frac{\xi_{\mathbf{k}+\mathbf{Q}}}{E_{\mathbf{k}}^{(L)}}\right)\right) \times\left(\frac{\Delta_{\mathbf{k}}^{2}}{q(\mathbf{k})}\right)\right\}\right],
\end{aligned}
$$

where $\xi_{\mathbf{k}+\mathbf{Q}}^{\prime}{ }^{2}=\left(\xi_{\mathbf{k}+\mathbf{Q}}^{2}+\Delta_{\mathbf{k}}^{(\mathrm{sc}) 2}\right)$, and $q(\mathbf{k})=\left(E_{\mathbf{k}}^{(U) 2}-E_{\mathbf{k}}^{(L) 2}\right)$. We find that the coherence factors $u_{\mathbf{k}}^{( \pm)^{2}}$ and $v_{\mathbf{k}}^{( \pm)^{2}}$ satisfy the sum rule $\left(u_{\mathbf{k}}^{(+)^{2}}+u_{\mathbf{k}}^{(-)^{2}}+v_{\mathbf{k}}^{(+)^{2}}+v_{\mathbf{k}}^{(-)^{2}}\right)=1$. The singleparticle spectral function in the DDW + DSC state, as before, is given by $A(\mathbf{k}, \omega)=2 \pi\left[u_{\mathbf{k}}^{(+)^{2}} \delta\left(\omega+E_{\mathbf{k}}^{(U)}\right)+u_{\mathbf{k}}^{(-)^{2}} \delta(\omega-\right.$ $\left.\left.E_{\mathbf{k}}^{(U)}\right)+v_{\mathbf{k}}^{(+)^{2}} \delta\left(\omega+E_{\mathbf{k}}^{(L)}\right)+v_{\mathbf{k}}^{(-)^{2}} \delta\left(\omega-E_{\mathbf{k}}^{(L)}\right)\right]$. Similarly, the spectral function $A(\mathbf{k}+\mathbf{Q}, \omega)$ is given by $A(\mathbf{k}+\mathbf{Q}, \omega)=$ $2 \pi\left[u_{\mathbf{k}+\mathbf{Q}}^{(+)}{ }^{2} \delta\left(\omega+E_{\mathbf{k}}^{(U)}\right)+u_{\mathbf{k}+\mathbf{Q}}^{(-)}{ }^{2} \delta\left(\omega-E_{\mathbf{k}}^{(U)}\right)+v_{\mathbf{k}+\mathbf{Q}}^{(+)}{ }^{2} \delta\left(\omega+E_{\mathbf{k}}^{(L)}\right)+\right.$ $\left.v_{\mathbf{k}+\mathbf{Q}}^{(-)} \delta\left(\omega-E_{\mathbf{k}}^{(L)}\right)\right]$. We find that, when the nesting property is satisfied, $u_{\mathbf{k}+\mathbf{Q}}^{(+)}{ }^{2}=u_{\mathbf{k}}^{(-)^{2}}, u_{\mathbf{k}+\mathbf{Q}}^{(-)}{ }^{2}=u_{\mathbf{k}}^{(+)^{2}}, v_{\mathbf{k}+\mathbf{Q}}^{(+)}{ }^{2}=v_{\mathbf{k}}^{(-)^{2}}$, and $v_{\mathbf{k}+\mathbf{Q}}^{(-)}{ }^{2}=v_{\mathbf{k}}^{(+)^{2}}$. In this situation, the sum $(A(\mathbf{k}, \omega)+A(\mathbf{k}+$ $\mathbf{Q}, \omega))=2 \pi\left[\left(u_{\mathbf{k}}^{(+)^{2}}+u_{\mathbf{k}}^{(-)^{2}}\right)\left(\delta\left(\omega+E_{\mathbf{k}}^{(U)}\right)+\delta\left(\omega-E_{\mathbf{k}}^{(U)}\right)\right)+\left(v_{\mathbf{k}}^{(+)^{2}}+\right.\right.$ $\left.\left.v_{\mathbf{k}}^{(-)^{2}}\right)\left(\delta\left(\omega+E_{\mathbf{k}}^{(L)}\right)+\delta\left(\omega-E_{\mathbf{k}}^{(L)}\right)\right)\right]$. As we shall see below, the $\operatorname{sum}(A(\mathbf{k}, \omega)+A(\mathbf{k}+\mathbf{Q}, \omega))$ will be required for the derivation of an expression for the thermodynamic potential following the Kadanoff-Baym approach $[47,48]$. The remaining Fourier coefficients $G_{\sigma}^{\prime}\left(k, \omega_{n}\right)$ and $\Gamma_{\sigma}\left(\mathbf{k}, \omega_{n}\right)$ which correspond to the DDW gap and the DSC gap, respectively, are given by

$$
\begin{aligned}
G_{\sigma}^{\prime}\left(k, \omega_{n}\right)= & -\left(\frac{D_{\mathbf{k}}^{\dagger}}{2 E_{\mathbf{k}}^{(U)}}\right) \\
& \times\left[\frac{\left\{\left(E_{\mathbf{k}}^{(U)}-\xi_{\mathbf{k}}\right) \times\left(E_{\mathbf{k}}^{(U)}-\xi_{\mathbf{k}+\mathbf{Q}}\right)-D_{\mathrm{eff}}(\mathbf{k})^{2}\right\}}{q(\mathbf{k})}\right] \\
& \times\left(i \omega_{n}+E_{\mathbf{k}}^{(U)}\right)^{-1}+\left(\frac{D_{\mathbf{k}}^{\dagger}}{2 E_{\mathbf{k}}^{(U)}}\right) \\
& \times\left[\frac{\left\{\left(E_{\mathbf{k}}^{(U)}+\xi_{\mathbf{k}}\right) \times\left(E_{\mathbf{k}}^{(U)}+\xi_{\mathbf{k}+\mathbf{Q}}\right)-D_{\mathrm{eff}}(\mathbf{k})^{2}\right\}}{q(\mathbf{k})}\right] \\
& \times\left(i \omega_{n}-E_{\mathbf{k}}^{(U)}\right)^{-1}+\left(\frac{D_{\mathbf{k}}^{\dagger}}{2 E_{\mathbf{k}}^{(L)}}\right)
\end{aligned}
$$




$$
\begin{aligned}
& \times\left[\frac{\left\{\left(E_{\mathbf{k}}^{(L)}-\xi_{\mathbf{k}}\right) \times\left(E_{\mathbf{k}}^{(L)}-\xi_{\mathbf{k}+\mathbf{Q}}\right)-D_{\text {eff }}(\mathbf{k})^{2}\right\}}{q(\mathbf{k})}\right] \\
& \times\left(i \omega_{n}+E_{\mathbf{k}}^{(L)}\right)^{-1}-\left(\frac{D_{\mathbf{k}}^{\dagger}}{2 E_{\mathbf{k}}^{(L)}}\right) \\
& \times\left[\frac{\left\{\left(E_{\mathbf{k}}^{(L)}+\xi_{\mathbf{k}}\right) \times\left(E_{\mathbf{k}}^{(L)}+\xi_{\mathbf{k}+\mathbf{Q}}\right)-D_{\text {eff }}(\mathbf{k})^{2}\right\}}{q(\mathbf{k})}\right] \\
& \times\left(i \omega_{n}-E_{\mathbf{k}}^{(L)}\right)^{-1}, \\
& \Gamma_{\sigma}\left(\mathbf{k}, \omega_{n}\right)=-\left(\frac{\Delta_{\mathbf{k}}^{(\mathrm{sc})}}{2 E_{\mathbf{k}}^{(U)}}\right)\left[\frac{\left(E_{\mathbf{k}}^{(U) 2}-\xi_{\mathbf{k}+\mathbf{Q}}^{2}-D_{\mathrm{eff}}(\mathbf{k})^{2}\right)}{\left(E_{\mathbf{k}}^{(U) 2}-E_{\mathbf{k}}^{(L) 2}\right)}\right] \\
& \times\left(i \omega_{n}+E_{\mathbf{k}}^{(U)}\right)^{-1}+\left(\frac{\Delta_{\mathbf{k}}^{(\mathrm{sc})}}{2 E_{\mathbf{k}}^{(U)}}\right) \\
& \times\left[\frac{\left(E_{\mathbf{k}}^{(U) 2}-\xi_{\mathbf{k}+\mathbf{Q}}^{2}-D_{\text {eff }}(\mathbf{k})^{2}\right)}{\left(E_{\mathbf{k}}^{(U) 2}-E_{\mathbf{k}}^{(L) 2}\right)}\right]\left(i \omega_{n}-E_{\mathbf{k}}^{(U)}\right)^{-1} \\
& +\left(\frac{\Delta_{\mathbf{k}}^{(\mathrm{sc})}}{2 E_{\mathbf{k}}^{(L)}}\right)\left[\frac{\left(E_{\mathbf{k}}^{(L) 2}-\xi_{\mathbf{k}+\mathbf{Q}}^{2}-D_{\mathrm{eff}}(\mathbf{k})^{2}\right)}{\left(E_{\mathbf{k}}^{(U) 2}-E_{\mathbf{k}}^{(L) 2}\right)}\right] \\
& \times\left(i \omega_{n}+E_{\mathbf{k}}^{(L)}\right)^{-1}-\left(\frac{\Delta_{\mathbf{k}}^{(\mathrm{sc})}}{2 E_{\mathbf{k}}^{(L)}}\right) \\
& \times\left[\frac{\left(E_{\mathbf{k}}^{(L) 2}-\xi_{\mathbf{k}+\mathbf{Q}}^{2}-D_{\mathrm{eff}}(\mathbf{k})^{2}\right)}{\left(E_{\mathbf{k}}^{(U) 2}-E_{\mathbf{k}}^{(L) 2}\right)}\right]\left(i \omega_{n}-E_{\mathbf{k}}^{(L)}\right)^{-1} .
\end{aligned}
$$

Upon replacing $D_{\mathbf{k}}$ and $D_{\mathbf{k}}^{\dagger}$, respectively, by $\left(i \Delta_{\mathbf{k}}\right)$ and $\left(-i \Delta_{\mathbf{k}}\right)\left(\right.$ and $\Delta_{\mathbf{k}}^{(\mathrm{sc})}=\Delta_{\mathbf{k}}^{\dagger}$ (sc) $)$, as in the pure $d$-wave case, the two Fourier coefficients $G_{\sigma}^{\prime}\left(k, \omega_{n}\right)$ and $\Gamma_{\sigma}\left(\mathbf{k}, \omega_{n}\right)$ lead to the DDW $\left(\Delta_{\mathbf{k}}\right)$ and DSC gap $\left(\Delta_{\mathbf{k}}^{(\mathrm{sc})}\right)$ equations while $G_{\sigma}\left(\mathbf{k}, \omega_{n}\right)$ leads to the equation for the chemical potential. With $G_{\sigma}^{\prime}\left(k, \omega_{n}\right)$, in view of the definition $D_{\mathbf{k}}^{\dagger} \equiv-\sum_{k^{\prime}, \sigma} U\left(\mathbf{k}, \mathbf{k}^{\prime}\right)\left\langle d_{\mathbf{k}^{\prime}, \sigma}^{\dagger} d_{\mathbf{k}^{\prime}+\mathbf{Q}, \sigma}\right\rangle$ and the first equation in (12), we obtain

$$
\begin{aligned}
D_{\mathbf{k}^{\prime}}^{\dagger}=\sum_{\mathbf{k}, \sigma} U\left(\mathbf{k}^{\prime}, \mathbf{k}\right)[ & \left(\frac{D_{\mathbf{k}}^{\dagger}}{2 q(\mathbf{k}) E_{\mathbf{k}}^{(U)}}\right) \\
\times & \left\{S_{\mathbf{k}-}^{(U)}\left(\exp \left(-\beta E_{\mathbf{k}}^{(U)}\right)+1\right)^{-1}\right. \\
& \left.-S_{\mathbf{k}+}^{(U)}\left(\exp \left(\beta E_{\mathbf{k}}^{(U)}\right)+1\right)^{-1}\right\} \\
& -\left(\frac{D_{\mathbf{k}}^{\dagger}}{2 q(\mathbf{k}) E_{\mathbf{k}}^{(L)}}\right) \\
\times & \left\{S_{\mathbf{k}-}^{(L)}\left(\exp \left(-\beta E_{\mathbf{k}}^{(L)}\right)+1\right)^{-1}\right. \\
& \left.\left.\quad-S_{\mathbf{k}+}^{(L)}\left(\exp \left(\beta E_{\mathbf{k}}^{(L)}\right)+1\right)^{-1}\right\}\right],
\end{aligned}
$$

where $S_{\mathbf{k} \pm}^{(j=U, L)} \equiv\left\{\left(E_{\mathbf{k}}^{(j)} \pm \xi_{\mathbf{k}}\right) \times\left(E_{\mathbf{k}}^{(j)} \pm \xi_{\mathbf{k}+\mathbf{Q}}\right)-D_{\text {eff }}(\mathbf{k})^{2}\right\}$. In the zero-temperature limit when nesting of the Fermi surface is near perfect, as in the square lattice with nearestneighbor hopping and a small second neighbor hopping $\left(\left(t_{2} / t_{1}\right)\right.$ small compared to unity), (13) may be written as $D_{\mathbf{k}^{\prime}}^{\dagger} \approx(1 / 2) \sum_{\mathbf{k}, \sigma} U\left(\mathbf{k}^{\prime}, \mathbf{k}\right)\left\{D_{\mathbf{k}}^{\dagger} /\left(\xi_{\mathbf{k}}^{2}+D_{\text {eff }}(\mathbf{k})^{2}\right)^{1 / 2}\right\}$. With the model interaction $U\left(\mathbf{k}^{\prime}, \mathbf{k}\right)=U_{1}\left(\cos k_{x} a-\cos k_{y} a\right)\left(\cos k_{x}^{\prime} a-\right.$ $\left.\cos k_{y}^{\prime} a\right)$, where $U_{1}$ is the repulsive coupling strength $\left(U_{1}>\right.$ $0)$, this equation assumes a simple form $1 \approx U_{1} \sum_{\mathbf{k}}\left[\left(\cos k_{x} a-\right.\right.$ $\left.\cos k_{y} a\right)^{2} /\left\{\xi_{\mathbf{k}}^{2}+\left(\Delta_{0}^{(\mathrm{PG})}(0)^{2}+\Delta_{0}^{(\mathrm{sc})}(0)^{2}\right)\left(\cos k_{x} a-\right.\right.$ $\left.\left.\cos k_{y} a\right)^{2}\right\}^{1 / 2}$. For the gap $\Delta_{\mathbf{k}}^{(\mathrm{sc})}$, on the other hand, we obtain

$$
\begin{aligned}
\Delta_{\mathbf{k}}^{(\mathrm{sc})}= & -\sum_{\mathbf{k}^{\prime}, \sigma} V\left(\mathbf{k}, \mathbf{k}^{\prime}\right)\left\{\frac{\Delta_{\mathbf{k}^{\prime}}^{(\mathrm{sc})}}{\left(2 q\left(\mathbf{k}^{\prime}\right) E_{\mathbf{k}^{\prime}}^{(U)}\right)}\right\} \\
& \times\left(E_{\mathbf{k}^{\prime}}^{(U) 2}-a\left(\mathbf{k}^{\prime}\right)\right) \tanh \left(\frac{\beta E_{\mathbf{k}^{\prime}}^{(U)}}{2}\right) \\
& +\sum_{\mathbf{k}^{\prime}, \sigma} V\left(\mathbf{k}, \mathbf{k}^{\prime}\right)\left\{\frac{\Delta_{\mathbf{k}^{\prime}}^{(\mathrm{sc})}}{\left(2 q\left(\mathbf{k}^{\prime}\right) E_{\mathbf{k}^{\prime}}^{(L)}\right)}\right\} \\
& \times\left(E_{\mathbf{k}^{\prime}}^{(L) 2}-a\left(\mathbf{k}^{\prime}\right)\right) \tanh \left(\frac{\beta E_{\mathbf{k}^{\prime}}^{(L)}}{2}\right),
\end{aligned}
$$

where $a(\mathbf{k}) \equiv\left(\xi_{\mathbf{k}+\mathbf{Q}}^{2}+D_{\text {eff }}(\mathbf{k})^{2}\right)$. In the zero-temperature case, for a nonnested dispersion, (14) reduces to

$$
\begin{aligned}
\Delta_{\mathbf{k}}^{(\mathrm{sc})}= & -\left(\frac{1}{2}\right) \sum_{\mathbf{k}^{\prime}, \sigma} V\left(\mathbf{k}, \mathbf{k}^{\prime}\right)\left\{\frac{\Delta_{\mathbf{k}^{\prime}}^{(\mathrm{sc})}}{\left(E_{\mathbf{k}^{\prime}}^{(U)}+E_{\mathbf{k}^{\prime}}^{(L)}\right)}\right\} \\
& \times\left\{1+\left(\frac{a\left(\mathbf{k}^{\prime}\right)}{E_{\mathbf{k}^{\prime}}^{(U)} E_{\mathbf{k}^{\prime}}^{(L)}}\right)\right\} .
\end{aligned}
$$

The near-nested dispersion yields $\Delta_{\mathbf{k}}^{(\mathrm{sc})} \approx$ $-(1 / 2) \sum_{\mathbf{k}^{\prime}, \sigma} V\left(\mathbf{k}, \mathbf{k}^{\prime}\right) \Delta_{\mathbf{k}^{\prime}}^{(\mathrm{sc})} \quad \times \quad\left[\mathrm{p}\left(\xi_{\mathbf{k}^{\prime}}^{2}+D_{\mathrm{eff}}\left(\mathbf{k}^{\prime}\right)^{2}\right)\right]^{-1}$ which is similar to the weak coupling BCS gap equation. With an appropriate attractive interaction $V\left(\mathbf{k}, \mathbf{k}^{\prime}\right)=-\left|V_{1}\right|\left(\cos k_{x} a-\cos k_{y} a\right)\left(\cos k_{x}^{\prime} a-\cos k_{y}^{\prime} a\right)$, where $V_{1}$ is the coupling strength, we find that the equation assumes a simple form $1 \approx\left|V_{1}\right| \sum_{\mathbf{k}}\left[\left(\cos k_{x} a-\cos k_{y} a\right)^{2} /\left\{\xi_{\mathbf{k}}^{2}+\right.\right.$ 
$\left.\left.\left(\Delta_{0}^{(\mathrm{PG})}(0)^{2}+\Delta_{0}^{(\mathrm{sc})}(0)^{2}\right)\left(\cos k_{x} a-\cos k_{y} a\right)^{2}\right\}^{1 / 2}\right]$. With the two gap equations combined, we obtain

$$
\begin{aligned}
1 \approx\left(\frac{1}{2}\right)\left(U_{1}+\left|V_{1}\right|\right) & \\
\times \sum_{\mathbf{k}}[ & \left(\cos k_{x} a-\cos k_{y} a\right)^{2} \\
\times & \left\{\xi_{\mathbf{k}}^{2}+\left(\Delta_{0}^{(\mathrm{PG})}(0)^{2}+\Delta_{0}^{(\mathrm{sc})}(0)^{2}\right)\right. \\
& \left.\left.\times\left(\cos k_{x} a-\cos k_{y} a\right)^{2}\right\}^{-1 / 2}\right] .
\end{aligned}
$$

We also obtain $U_{1} \approx\left|V_{1}\right|$ as the sum $\sum_{\mathbf{k}}\left[\left(\cos k_{x} a-\cos k_{y} a\right)^{2} \times\left\{\xi_{\mathbf{k}}^{2}+\left(\Delta_{0}^{(\mathrm{PG})}(0)^{2}+\Delta_{0}^{(\mathrm{sc})}(0)^{2}\right) \times\right.\right.$ $\left.\left.\left(\cos \left(k_{x} a\right)-\cos \left(k_{y} a\right)\right)^{2}\right\}^{-1 / 2}\right]$ is not zero. Since $U_{1}$ has its origin in the coulombic interaction [9-11], the condition $U_{1} \approx\left|V_{1}\right|$ obtained hints at the possibility of noninvolvement of the electron-phonon type interaction in the hole-doped cuprates. Upon modeling the functional dependence of the pairing interactions and order parameter as $U\left(\mathbf{k}, \mathbf{k}^{\prime}\right) \rightarrow$ $U_{1} \cos (2 \varphi) \cos \left(2 \varphi^{\prime}\right), V\left(\mathbf{k}, \mathbf{k}^{\prime}\right) \rightarrow-\left|V_{1}\right| \cos (2 \varphi) \cos \left(2 \varphi^{\prime}\right)$, and squared order parameter $\Delta_{\mathbf{k}}(T=0)^{2}=\left(\Delta_{0}^{(\mathrm{PG})}(T=0)^{2}+\right.$ $\left.\Delta_{0}^{(\mathrm{sc})}(T=0)^{2}\right)\left(\cos k_{x} a-\cos k_{y} a\right)^{2} \rightarrow \Delta_{0}(0)^{2} \cos ^{2}(2 \varphi)$, where $\varphi \equiv \operatorname{arc}\left(\tan \left(k_{y} / k_{x}\right)\right)$ and $\left(k_{x}, k_{y}\right)$ belong to the first Brillouin zone (BZ), we may write the gap equation (16) as

$$
\begin{aligned}
1 \approx & g\left(k_{F}\right) \int_{0}^{2 \pi}\left(\frac{d \varphi}{2 \pi}\right) \\
& \times\left(\int_{0}^{+1} d \xi\left[\frac{\cos ^{2}(2 \varphi)}{\sqrt{\left(\xi^{2}+\left(\Delta_{0}(0) / \hbar \omega_{c}\right)^{2} \cos ^{2}(2 \varphi)\right)}}\right]\right) \\
= & g\left(k_{F}\right) \int_{0}^{2 \pi}\left(\frac{d \varphi}{2 \pi}\right) \cos ^{2}(2 \varphi) \\
& \times \log \left|\frac{\left.\sqrt{\left(\left(\Delta_{0}(0) / \hbar \omega_{c}\right)^{2} \cos ^{2}(2 \varphi)+1\right)}+1\right\}}{\left\{\left(\Delta_{0}(0) / \hbar \omega_{c}\right) \times \cos (2 \varphi)\right\}}\right|,
\end{aligned}
$$

where the dimensionless quantity $g\left(k_{F}\right)=(D / 2)\left(U_{1}+\left|V_{1}\right|\right)$ and $\Delta_{0}(T=0)=\left(\Delta_{0}^{(\mathrm{PG})}(T=0)^{2}+\Delta_{0}^{(\mathrm{sc})}(T=0)^{2}\right)^{1 / 2}$. In writing energy integrations in the gap equation, we have assumed an arbitrary energy cut-off " $\hbar \omega_{c}$ " less than the Fermi energy. This facilitates integration over a length larger than $k_{F}^{-1}$. The quantity $D$ with dimensions (Energy) ${ }^{-1}$, which will be assumed to be a constant (see Figure 16), is the density of energy states. In the weak coupling limit $g\left(k_{F}\right) \ll 1$, for the special situation $\varphi \rightarrow 0$, we obtain $\left(\Delta_{0}(0) / \hbar \omega_{c}\right) \approx$ $2 \exp \left(-1 / g\left(k_{F}\right)\right)$. This is reminiscent of the corresponding result of the BCS theory for the "conventional superconductors." For the unconventional superconductor under consideration here, we adopt the simple strategy of assigning numerical values to $\left(\Delta_{0}(0) / \hbar \omega_{c}\right)$ and performing the integration in (17), by discretizing the integral, to obtain $g\left(k_{F}\right)$. The $2 \mathrm{D}$ graph in Figure 3 displays the outcome. We find that the quantity $\left(\Delta_{0}(0) / \hbar \omega_{c}\right)$ is an increasing function of $g\left(k_{F}\right)$. Though the quantitative aspect of the result may be an artefact of the model adopted, qualitatively the exercise underscores the fact that reasonable strong-coupling solution for the order parameter amplitude $\Delta_{0}(T=0)$ for a superconductor of $d$-wave variety is available within the BCS framework. Now at all temperatures above $T=0 \mathrm{~K}$, there is a finite possibility of finding electrons in the nonsuperconducting state. At finite temperature, the occupation of the excited oneelectron state $E_{k}=\sqrt{\left(\xi_{\mathbf{k}}^{2}+\Delta_{\mathbf{k}}(T)^{2}\right)}$ obeys the Fermi statistics with the Fermi distribution ( $\left.\exp \beta E_{k}+1\right)^{-1}$. Equation (17) is, therefore, replaced by

$$
\begin{aligned}
1 \approx & g\left(k_{F}\right) \int_{0}^{2 \pi}\left(\frac{d \varphi}{2 \pi}\right) \\
& \times\left(\int_{0}^{+1} d \xi\left[\frac{\cos ^{2}(2 \varphi)}{\mathrm{p}\left(\xi^{2}+\left(\Delta_{0} / \hbar \omega_{c}\right)^{2} \cos ^{2}(2 \varphi)\right)}\right]\right) \\
& \times\left(1-2 f\left(T, \xi,\left(\frac{\Delta_{0}}{\hbar \omega_{c}}\right), \varphi\right)\right),
\end{aligned}
$$

where $f\left(T, \xi,\left(\Delta_{0} / \hbar \omega_{c}\right), \varphi\right)=\left(\exp \left\{\beta \hbar \omega_{c}\right.\right.$ $\left.\left.\left(\sqrt{\left(\xi^{2}+\left(\Delta_{0} / \hbar \omega_{c}\right)^{2} \cos ^{2}(2 \varphi)\right)}\right)\right\}+1\right)^{-1}$. We assume that $\Delta_{0}(T=0)^{2}=\left(\Delta_{0}^{(\mathrm{PG})}(T=0)^{2}+\Delta_{0}^{(\mathrm{sc})}(T=0)^{2}\right) \approx \Delta_{0}^{(\mathrm{sc})}(T=0)^{2}$ which makes sense if the DDW and DSC are noncooperating/competing orders (see Section 4). In that case, one may regard that the factor of 2 multiplying the Fermi function appears because either one of the states $\mathbf{k}$ or -k may be occupied. One can derive the equation for the critical temperature $T_{c}$ readily from above:

$$
2=g\left(k_{F}\right)\left(\int_{0}^{+1} d \xi\left(\frac{1}{\xi}\right) \tanh \left(\frac{\xi \hbar \omega_{c}}{2 k_{B} T_{c}}\right)\right.
$$

Equation (19) immediately gives $k_{B} T_{c}=$ $0.57 \hbar \omega_{c} \exp \left(-1 / g\left(k_{F}\right)\right)$. With the aid of data available (for $g\left(k_{F}\right)=1.0$ (strong coupling) we have $\left(\Delta_{0} / \hbar \omega_{c}\right)=0.5$ ) from Figure 3, we then obtain the ratio $\left(2 \Delta_{0} / k_{B} T_{c}\right) \approx 4.8$. This value is quite different from the experimental value $[19,49]$, where $T_{c}$ is approximately related both to the gap $(G)$ and to the extension of Fermi arcs by $2 \mathrm{G}(4 \alpha / \pi)=4.3 k_{B} T_{c}$ where the angle $\alpha$ spans the half-Fermi arc as measured 
from the nodal direction. It may be relevant to note that the anomalous pairing

$$
\begin{aligned}
\left\langle d_{\mathbf{k}, \sigma}^{\dagger} d_{-\mathbf{k}-\mathbf{Q},-\sigma}^{\dagger}\right\rangle= & \left\{\frac{-D_{k}^{\dagger} \Delta_{\mathbf{k}}^{(\mathrm{sc})}\left(\xi_{\mathbf{k}}+\xi_{\mathbf{k}+\mathbf{Q}}\right)}{2 q(\mathbf{k}) E_{\mathbf{k}}^{(U)}}\right\} \\
& \times \tanh \left(\frac{\beta E_{\mathbf{k}}^{(U)}}{2}\right) \\
& +\left\{\frac{-D_{k}^{\dagger} \Delta_{\mathbf{k}}^{(\mathrm{sc})}\left(\xi_{\mathbf{k}}+\xi_{\mathbf{k}+\mathbf{Q}}\right)}{2 q(\mathbf{k}) E_{\mathbf{k}}^{(L)}}\right\} \\
& \times \tanh \left(\frac{\beta E_{\mathbf{k}}^{(L)}}{2}\right)
\end{aligned}
$$

is required to be nonzero for the validity of the results, such as (5) and (6) and the four Bogoluibov bands $E_{\mathbf{k}}\left(T<T_{c}\right)=$ $\pm E^{(U, L)}(\mathbf{k})$, obtained above. This is possible only when the nesting is absent, that is, $\left(\xi_{\mathbf{k}}+\xi_{\mathbf{k}+\mathbf{Q}}\right) \neq 0$. Thus, the plot in Figure 3 and the result $\left(2 \Delta_{0} / k_{B} T_{c}\right) \approx 4.8$ obtained are only qualitatively accurate. This is why we have solved the coupled equations (13) and (14) numerically assuming the specific form for the interactions $U\left(\mathbf{k}, \mathbf{k}^{\prime}\right)$ and $V\left(\mathbf{k}, \mathbf{k}^{\prime}\right)$ given above for the nonnested dispersion together with the equation to determine the chemical potential $(\mu)$ obtainable from (11). The equation, according to the Luttinger rule, is given by

$$
\begin{aligned}
(1+p)=\left(\frac{N_{s}}{2}\right)^{-1} \sum_{k^{\prime}}[ & u_{\mathbf{k}^{\prime}}^{(+)^{2}} \times\left(\exp \left(-\beta E_{\mathbf{k}^{\prime}}^{(U)}\right)+1\right)^{-1} \\
& +u_{\mathbf{k}^{\prime}}^{(-)^{2}} \times\left(\exp \left(\beta E_{\mathbf{k}^{\prime}}^{(U)}\right)+1\right)^{-1} \\
+ & v_{\mathbf{k}^{\prime}}^{(+)^{2}} \times\left(\exp \left(-\beta E_{\mathbf{k}^{\prime}}^{(L)}\right)+1\right)^{-1} \\
& \left.+v_{\mathbf{k}^{\prime}}^{(-)^{2}} \times\left(\exp \left(\beta E_{\mathbf{k}^{\prime}}^{(L)}\right)+1\right)^{-1}\right],
\end{aligned}
$$

where $p$ is the hole-doping level, and $N_{s}$ is the number of unit cells in the $\mathbf{k}$-space. The $\mathbf{k}$-summations in these equations, as before, will be replaced by the integration $\int d(\mathbf{k} a) \rho_{\mathrm{Fermi}}(\mathbf{k}) \rightarrow \int_{-\pi}^{+\pi} d\left(k_{x} a\right) / 2 \pi \int_{-\pi}^{+\pi} d\left(k_{y} a\right) / 2 \pi \rho_{\mathrm{Fermi}}(\mathbf{k})$, where $\rho_{\text {Fermi }}(\mathbf{k})$ stands for the Fermi energy density of states (DOSs). For example, for the doping level 9.94\% and temperatures $T=60 \mathrm{~K}$ and $20 \mathrm{~K}$, respectively, solving these equations simultaneously, we have found $\left(\mu / t_{1}=-0.0018\right.$, $\left.\Delta_{0}^{(\mathrm{PG})} / t_{1}=0.0240, \Delta_{0}^{(\mathrm{sc})} / t_{1}=0.0150\right)$, and $\left(\mu / t_{1}=-0.0009\right.$, $\left.\Delta_{0}^{(\mathrm{PG})} / t_{1}=0.0170, \Delta_{0}^{(\mathrm{sc})} / t_{1}=0.0294\right)$ for $\left(\left(t_{2} / t_{1}\right) \approx 0.4\right.$, $\left.\left(t_{3} / t_{1}\right)=0.0005\right)$.

The above-mentioned exercise leads to the graphical representations of $\varepsilon_{k}^{(U, L)}$ in the pure DDW state, $\pm \dot{\varepsilon}(\mathbf{k})$ in the pure DSC state, and $\pm E^{(U, L)}(\mathbf{k})$ in the coexistent DDW and DSC states. In Figure 4(a), we have shown the plot of $\varepsilon_{k}^{(U, L)}$ along the antinodal cut $(-\pi, \pi)-(0, \pi)-(\pi, \pi)$, while in
Figures $4(\mathrm{~b})$ and $4(\mathrm{c})$ we have shown the plot of $\pm \dot{\varepsilon}(\mathbf{k})$ and $\pm E^{(U, L)}(\mathbf{k})$, respectively. In order to highlight their special features, namely, the particle-hole asymmetry (symmetry) for $\varepsilon_{\mathbf{k}}^{(U, L)}$ and $\pm \varepsilon_{\mathbf{k}}^{(U, L)}( \pm \dot{\varepsilon}(\mathbf{k}))$, we note that the optical and electronic phenomena in solids arise from the behavior of electrons and holes (unoccupied states in a filled electron sea). Electron-hole symmetry can often be invoked as a simplifying description, which states that electrons with energy above the Fermi sea behave the same as holes below the Fermi energy. In semiconductors, for example, electronhole symmetry is generally absent because the energy-band structure of the conduction band differs from the valence band. In Figure 4(a), we find easy to notice that particlehole asymmetry as the upper band is parabolic while the lower band is characterized by $\operatorname{dip}$ at $(0, \pi)$ and the socalled back-bending momentum at $(-0.45, \pi)$ and $(0.45, \pi)$. A plot of the upper and the lower bands $\pm \dot{\varepsilon}(\mathbf{k})$ in Figure 4(b) indicates perfect electron-hole symmetry as both the upper and lower bands are parabolic. In Figure 4(c), we have shown a plot of the upper bands $E_{k}^{(U, L)}$ and the lower bands $-E_{k}^{(U, L)}$ in the DDW-DSC state once again along the antinodal cut $(-\pi, \pi)-(0, \pi)-(\pi, \pi)$. The shoulder-type feature of the dispersion $-E_{k}^{(L)}$, indicated by double-headed arrows, also exists in the experimental data of Hashimoto et al. [24]. The bands $\left(-E_{k}^{(U, L)}\right)$ below the Fermi energy are the reflected ones of those above the Fermi energy $\left(E_{k}^{(U, L)}\right)$. We notice that (i) in the presence of superconducting gap the elementary excitations are the Bogoluibov quasiparticles which mix electron and hole states, and (ii) as a manifestation of interplay of DDW and DSC the band $\left(-E_{k}^{(L)}\right)$ no more peak at $k_{F}(0, \pi)$ but rather at the back-bending momentum position as shown due to the presence of a particle-hole asymmetric pseudogap. It must be noted that the Bogoluibov quasiparticle band features, agreeable with $d$-wave BCS theory, observed in the superconducting state here have been reported time and again in the ARPES experiments [14, 24, 50-52].

The particle-hole asymmetry of $\varepsilon_{\mathbf{k}}^{(U, L)}$ and $\pm E_{\mathbf{k}}^{(U, L)}$ could also be visualized through the corresponding contour plots in the first Brillouin zone (BZ) shown in Figure 5. In Figures 5(a) and $5(\mathrm{~b})$, respectively, we have shown plots of $\varepsilon_{\mathbf{k}}^{(U)}$ and $\varepsilon_{\mathbf{k}}^{(L)}$. The band-maxima in $\varepsilon_{\mathbf{k}}^{(U)}$ occur at the points $( \pm \pi, \pm \pi)$ and $(0,0)$ whereas those in $\varepsilon_{\mathbf{k}}^{(L)}$ occur at the points $( \pm \pi / 2, \pm \pi / 2)$. Since the electrons with energy above the Fermi sea do not behave the same as the holes below the Fermi energy, we have the particle-hole asymmetry in the pure DDW state. In Figures 5(c) and 5(d), respectively, we have shown $\left(-E_{\mathbf{k}}^{(U)}\right)$ and $\left(-E_{\mathbf{k}}^{(L)}\right)$. Since the bands $E_{\mathbf{k}}^{(U, L)}$ above the Fermi energy are the reflected ones of those below the Fermi energy, that is, $\left(-E_{\mathbf{k}}^{(U, L)}\right)$, we have not shown $E_{\mathbf{k}}^{(U)}$ and $E_{\mathbf{k}}^{(L)}$ here. In fact, the band-maxima in $E_{\mathbf{k}}^{(U)}$ occur at the points $( \pm \pi, \pm \pi)$ and $(0,0)$ whereas those in $E_{\mathbf{k}}^{(L)}$ occur at the points $( \pm \pi / 2, \pm \pi / 2)$. On the other hand, the band-maxima in $\left(-E_{\mathbf{k}}^{(U)}\right)$ occur at the antinodal points $( \pm \pi, 0)$ and $(0, \pm \pi)$ while the band-minima in $\left(-E_{\mathbf{k}}^{(L)}\right)$ occur at the nodal points $( \pm \pi / 2, \pm \pi / 2)$. Since the Bogoluibov quasiparticles here with energy above the Fermi 


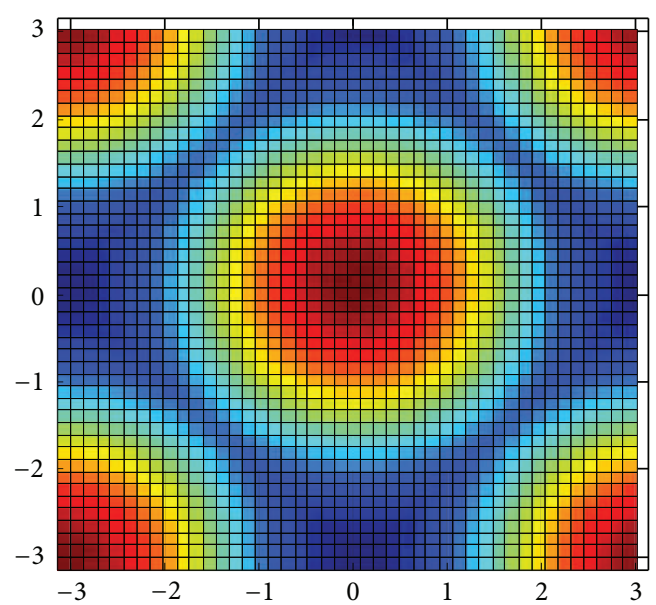

(a)

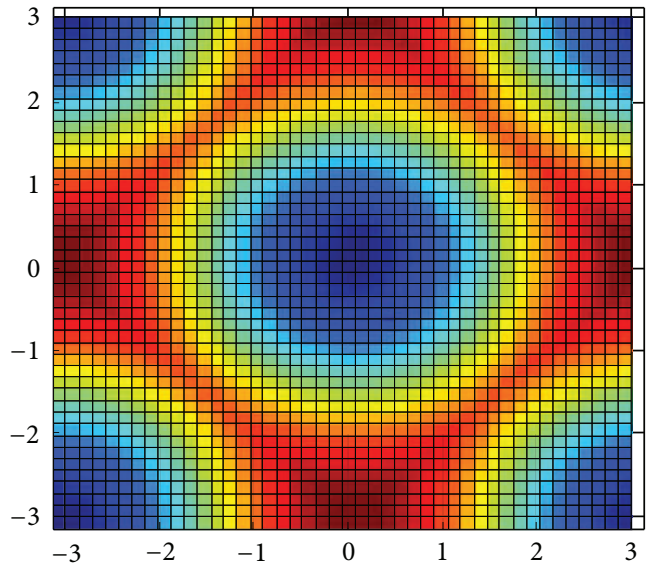

(c)

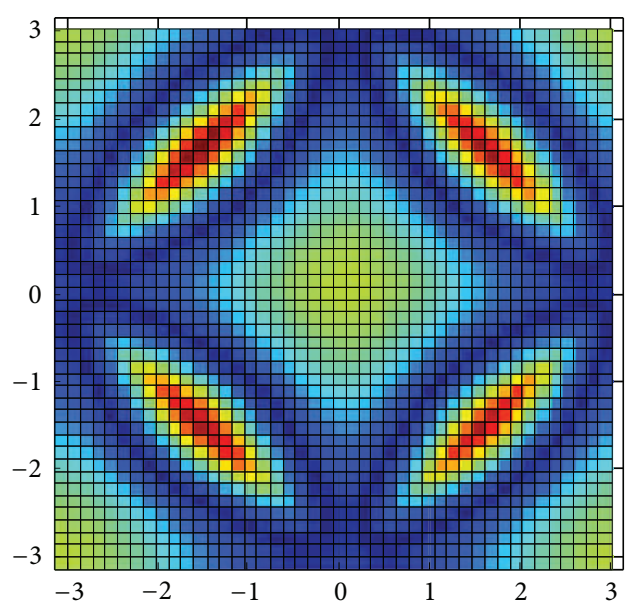

(b)

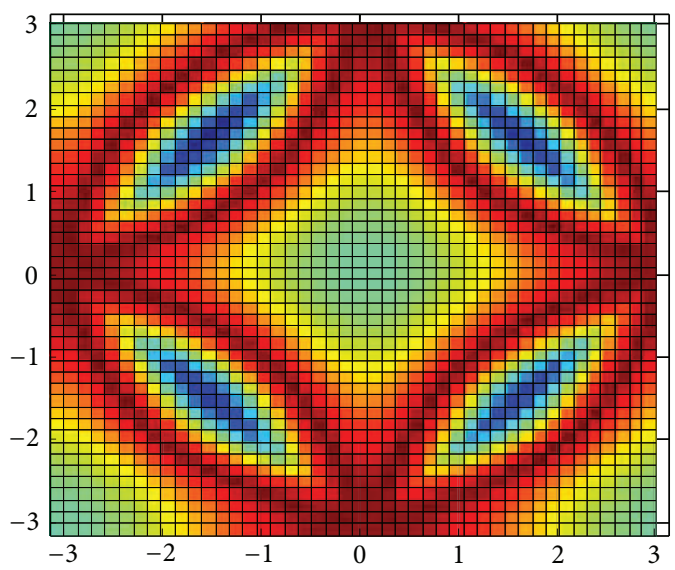

(d)

FIGURE 5: (i) The contour plots of the upper band $\varepsilon_{k}^{(U)}$ (a) and the lower band $\varepsilon_{k}^{(L)}$ (b) in the pure DDW state (doping level 9.94\%) on the first Brillouin zone (BZ). The wave vector component $\left(k_{x} a\right)$ is plotted along the horizontal direction while $\left(k_{y} a\right)$ is plotted along the vertical direction. The scale of the plot in (a) is from 0 to 8 whereas in (b) it is from 0 to -2 . The band-maxima in (a) occur at the points ( $\pm \pi$, $\pm \pi)$ and $(0,0)$. The band-maxima in (b) occur at the nodal points $( \pm \pi / 2, \pm \pi / 2)$. (ii) The contour plots of the band $\left(-E_{\mathbf{k}}^{(U)}\right)(\mathrm{c})$ and the band $\left(-E_{\mathbf{k}}^{(L)}\right)$ (b) in the DDW + DSC state (doping level 9.94\%) on the first Brillouin zone (BZ). The scale of the plot in (c) is from 0 to -8 while in (d) it is from 0 to -2 . The band-maxima in (c) occur at the antinodal points $( \pm \pi, 0)$ and $(0, \pm \pi)$. The band-minima in (d) occur at the nodal points $( \pm \pi / 2, \pm \pi / 2)$. The nodal quasiparticles correspond to the cone-like structure.

sea do not behave the same as those below the Fermi energy, we have clear particle-hole asymmetry in the DDW + DSC state as well.

\section{Thermodynamics and Transport Properties in PG Phase}

3.1. Thermodynamic Potential. We wish to obtain now expressions for the entropy and the specific heat in closed form for the DDW + SC state. For this purpose, it is convenient to define a thermodynamic potential $\Omega(\lambda)$ in terms of the Hamiltonian $H(\lambda)=\lambda H$ where $\lambda$ is a variable and $H$ is given by (2). One can write $\Omega(\lambda)-\Omega(0)=\int(d \lambda / \lambda)\langle H(\lambda)\rangle_{\lambda}$, where $\Omega(0)$ is an integration constant and the angular brackets $\langle\cdots\rangle_{\lambda}$ denote thermodynamic average calculated with $H(\lambda)$. The system under consideration corresponds to $\Omega(\lambda=$
1). Following the Kadanoff-Baym approach [47, 48], one can write a relation between the thermodynamic potential and the spectral functions $A_{\lambda}(k, \sigma, \omega)$, corresponding to the Matsubara propagators $G_{\sigma}^{\lambda}\left(\mathbf{k}, \omega_{n}\right), G_{\sigma}^{\lambda}\left(\mathbf{k}+\mathbf{Q}, \omega_{n}\right)$, and so forth as

$$
\begin{aligned}
& \Omega(\lambda)-\Omega(0) \\
&=\int\left(\frac{d \lambda}{\lambda}\right) \sum_{k, \sigma} \int_{-\infty}^{+\infty}\left(\frac{d \omega}{2 \pi}\right)\left(e^{\beta \omega}+1\right)^{-1} \\
& \times\left[\omega\left\{A_{\lambda}(\mathbf{k}, \sigma, \omega)+A_{\lambda}(\mathbf{k}+\mathbf{Q}, \sigma, \omega)\right\}\right. \\
&\left.+\Delta_{\mathbf{k}}^{(\mathrm{sc})} B_{\lambda}(\mathbf{k}, \sigma, \omega)+\Delta_{\mathbf{k}}^{\dagger(\mathrm{sc})} C_{\lambda}(\mathbf{k}, \sigma, \omega)\right],
\end{aligned}
$$


where the spectral functions $A_{\lambda}(\mathbf{k}, \sigma, \omega), A_{\lambda}(\mathbf{k}+\mathbf{Q}, \sigma, \omega)$, $B_{\lambda}(\mathbf{k}, \sigma, \omega)$, and $C_{\lambda}(\mathbf{k}, \sigma, \omega)$ are given by

$$
\begin{gathered}
A_{\lambda}(\mathbf{k}, \sigma, \omega)=i\left[\left.G_{\sigma}^{\lambda}\left(\mathbf{k}, \omega_{n}\right)\right|_{i \omega_{n}=\omega+i 0^{+}}\right. \\
\left.-\left.G_{\sigma}^{\lambda}\left(\mathbf{k}, \omega_{n}\right)\right|_{i \omega_{n}=\omega-i 0^{+}}\right], \\
A_{\lambda}(\mathbf{k}+\mathbf{Q}, \sigma, \omega)=i\left[\left.G_{\sigma}^{\lambda}\left(\mathbf{k}+\mathbf{Q}, \omega_{n}\right)\right|_{i \omega_{n}=\omega+i 0^{+}}\right. \\
\left.\quad-\left.G_{\sigma}^{\lambda}\left(\mathbf{k}+\mathbf{Q}, \omega_{n}\right)\right|_{i \omega_{n}=\omega-i 0^{+}}\right], \\
B_{\lambda}(\mathbf{k}, \sigma, \omega)=i\left[\left.\Gamma_{\sigma}^{\lambda}\left(\mathbf{k}, \omega_{n}\right)\right|_{i \omega_{n}=\omega+i 0^{+}}\right. \\
\left.\quad-\left.\Gamma_{\sigma}^{\lambda}\left(\mathbf{k}, \omega_{n}\right)\right|_{i \omega_{n}=\omega-i 0^{+}}\right], \\
C_{\lambda}(\mathbf{k}, \sigma, \omega)=i\left[\left.F_{\sigma}^{\lambda}\left(\mathbf{k}, \omega_{n}\right)\right|_{i \omega_{n}=\omega+i 0^{+}}\right. \\
\left.-\left.F_{\sigma}^{\lambda}\left(\mathbf{k}, \omega_{n}\right)\right|_{i \omega_{n}=\omega-i 0^{+}}\right] .
\end{gathered}
$$

Here $G_{\sigma}^{\lambda}(\mathbf{k}, \tau)=-\left\langle T\left\{d_{\mathbf{k}, \sigma}(\tau) d_{\mathbf{k}, \sigma}^{\dagger}(0)\right\}\right\rangle_{\lambda}, \Gamma_{\sigma}^{\lambda}(\mathbf{k}, \tau)=$ $-\left\langle T\left\{d_{-\mathbf{k},-\sigma}^{\dagger}(\tau) d_{\mathbf{k}, \sigma}^{\dagger}(0)\right\}\right\rangle_{\lambda}, F_{\sigma}^{\lambda}(\mathbf{k}, \tau)=-\left\langle T\left\{d_{\mathbf{k}, \sigma}(\tau) d_{-\mathbf{k},-\sigma}(0)\right\}\right\rangle_{\lambda}$, and $G_{\sigma}^{\lambda}(\mathbf{k}+\mathbf{Q}, \tau)=-\left\langle T\left\{d_{k+Q, \sigma}(\tau) d_{k+Q, \sigma}^{\dagger}(0)\right\}\right\rangle_{\lambda}$. Besides, $G_{\sigma}^{\lambda}\left(\mathbf{k}+\mathbf{Q}, \omega_{n}\right)=\int_{0}^{\beta} d \tau e^{i \omega n \tau} G_{\sigma}^{\lambda}(\mathbf{k}+\mathbf{Q}, \tau), F_{\sigma}^{\lambda}\left(k, \omega_{n}\right)=$ $\int_{0}^{\beta} d \tau e^{i \omega n \tau} F_{\sigma}^{\lambda}(k, \tau)$, and so on. Since we already have calculated $G_{\sigma}^{\lambda}\left(\mathbf{k}, \omega_{n}\right)$ and $\Gamma_{\sigma}^{\lambda}\left(\mathbf{k}, \omega_{n}\right)$, we shall be able to calculate the spectral functions $A_{\lambda}(\mathbf{k}, \sigma, \omega)$ and $B_{\lambda}(\mathbf{k}, \sigma, \omega)$. To calculate $A_{\lambda}(\mathbf{k}+\mathbf{Q}, \sigma, \omega)$, we use the result

$$
\begin{aligned}
G_{\sigma}\left(\mathbf{k}+\mathbf{Q}, \omega_{n}\right)=\{ & u_{\mathbf{k}+\mathbf{Q}}^{(+)}{ }^{2}\left(i \omega_{n}+E_{\mathbf{k}}^{(U)}\right)^{-1}+u_{\mathbf{k}+\mathbf{Q}}^{(-)}{ }^{2} \\
& \times\left(i \omega_{n}-E_{\mathbf{k}}^{(U)}\right)^{-1}+v_{\mathbf{k}+\mathbf{Q}}^{(+)}\left(i \omega_{n}+E_{\mathbf{k}}^{(L)}\right)^{-1} \\
& \left.+v_{\mathbf{k}+\mathbf{Q}}^{(-)}{ }^{2}\left(i \omega_{n}-E_{\mathbf{k}}^{(L)}\right)^{-1}\right\},
\end{aligned}
$$

where the coherence factors $\left(u_{\mathbf{k}+\mathbf{Q}}^{( \pm)}{ }^{2}, v_{\mathbf{k}+\mathbf{Q}}^{( \pm}{ }^{2}\right)$ may be written down using (11). In fact, this has already been done below (11). Since in the pure $d$-wave case $\Delta_{\mathbf{k}}^{\dagger(\text { sc) }}=\Delta_{\mathbf{k}}^{(\text {sc) }}$, we need not calculate $C_{\lambda}(\mathbf{k}, \sigma, \omega)$ as this is given by $B_{\lambda}(\mathbf{k}, \sigma, \omega)$. In the pure DDW state, we find that the thermodynamic potential is given by the expression

$$
\begin{gathered}
\Omega(T, \mu)=\Omega_{0}-2 \beta^{-1} \int d(\mathbf{k} a) \rho_{\text {Fermi }}(\mathbf{k}) \\
\sum_{j(=U, L)}\left\{\ln \cosh \left(\frac{\beta \varepsilon_{\mathbf{k}}^{(j)}}{2}\right)\right\}, \\
\Omega_{0}=\int d(\mathbf{k} a) \rho_{\text {Fermi }}(\mathbf{k}) \sum_{j(=U, L)} \varepsilon_{\mathbf{k}}^{(j)} .
\end{gathered}
$$

Using this expression, we shall calculate the entropy $(S)$ and the electronic specific heat $\left(C_{\mathrm{el}}\right)$. However, this requires the calculation of the Fermi energy density of states (DOSs) which we wish to discuss first before we calculate $\left(S, C_{\mathrm{el}}\right)$.

3.2. Elastic Scattering by Impurities and Quasiparticle Lifetime. We now wish to calculate the lifetimes of the electron and hole quasiparticle below and show that the former is short-lived compared to the latter. We also wish to link this important fact with the entropy, the electronic specific heat and the thermal conductivity. In order to calculate the quasiparticle lifetime (QPLT), we need to consider the effect of elastic scattering by impurities. This involves calculation of self-energy $\Sigma\left(\mathbf{k}, \omega_{n}\right)$, involving the momentum and the Matsubara frequencies $\omega_{n}$. The self-energy alters the singleparticle excitation spectrum in a fundamental way. A few diagrams contributing to the self-energy are shown in Figure 6 . The unsmooth lines carry momentum but no energy as the scattering is assumed to be elastic. The total momentum entering each impurity vertex, depicted by a dark circle, is zero. We assume that impurities are alike, distributed randomly, and contribute a potential term $V(\mathbf{r}=\mathbf{R}+$ $z \mathbf{k})=\sum_{i} u\left(\mathbf{r}-\mathbf{r}_{i}\right)$, where $u\left(\mathbf{r}-\mathbf{r}_{i}\right)$ is the potential due to a single impurity at $\mathbf{r}_{\mathbf{i}}=\mathbf{R}_{\mathbf{i}}+z \mathbf{k}$ for a given $z$ and $\mathbf{R}=x \mathbf{i}+y \mathbf{j}$. The term $u\left(\mathbf{r}-\mathbf{r}_{i}\right)$ is expanded in a Fourier series: $u\left(\mathbf{r}-\mathbf{r}_{i}\right)=\sum_{\mathbf{q}, i} u(|\mathbf{q}|) \exp \left[i \mathbf{q} \cdot\left(\mathbf{r}-\mathbf{r}_{i}\right)\right]$. We first consider only the contribution of Figure 6(a). Assuming the scattering by impurities weak, we may write it as $\Sigma^{(1)}\left(\mathbf{k}, \omega_{n}\right)=N_{j} \sum_{\mathbf{k}^{\prime}}\left|u\left(\mathbf{k}-\mathbf{k}^{\prime}\right)\right|^{2} G\left(\mathbf{k}^{\prime}, \omega_{n}\right)=\Sigma_{0}^{(1)}\left(\mathbf{k}, \omega_{n}\right)+\Sigma_{e}$ where

$$
\begin{aligned}
& \Sigma_{0}^{(1)}\left(\mathbf{k}, \omega_{n}\right)=-N_{j} \sum_{\mathbf{k}^{\prime}}\left|u\left(\mathbf{k}-\mathbf{k}^{\prime}\right)\right|^{2}\left(i \omega_{n}\right) \\
& \times \int_{-\infty}^{+\infty} d \varepsilon \rho(\varepsilon) \times\left[u_{\mathbf{k}^{\prime}}^{2}\left(\omega_{n}^{2}+\varepsilon_{\mathbf{k}^{\prime}}^{(U) 2}\right)^{-1}\right. \\
&\left.+v_{\mathbf{k}^{\prime}}^{2}\left(\omega_{n}^{2}+\varepsilon_{\mathbf{k}^{\prime}}^{(L) 2}\right)^{-1}\right],
\end{aligned}
$$

$N_{j}$ is the impurity concentration, $u\left(\mathbf{k}-\mathbf{k}^{\prime}\right)$ characterizes the momentum-dependent impurity potential, and $\Sigma_{e}<0$ is the part of the first-order contribution which redefines the chemical potential (see the first equation in (29) below). To evaluate the integrals in (26), such as $\int_{-\infty}^{+\infty} d \varepsilon \rho(\varepsilon)\left(\omega_{n}^{2}+\varepsilon_{\mathbf{k}}^{(j) 2}\right)^{-1}$, we assume $\rho(\varepsilon)=\rho_{0} \delta\left(\varepsilon-\varepsilon_{\mathbf{k}}^{(j)}\right)$ where we take a broad bandwidth, say, $\left(10 t_{1}\right) \sim 1.65 \mathrm{eV}$ which gives $\rho_{0}=\left(10 t_{1}\right)^{-1} \sim$ $0.6(\mathrm{eV})^{-1}$ We, thus, obtain $G\left(\mathbf{k}, \omega_{n}\right) \approx-\rho_{0}\left(i \omega_{n}\right)\left(\pi /\left|\omega_{n}\right|\right)$, and

$$
\Sigma_{0}^{(1)}\left(\mathbf{k}, \omega_{n}\right)=-N_{j} \rho_{0}\left(i \omega_{n}\right) \sum_{k^{\prime}}\left|u\left(\mathbf{k}-\mathbf{k}^{\prime}\right)\right|^{2}\left(\frac{\pi}{\left|\omega_{n}\right|}\right) .
$$

We may write the right-hand side of (27) as $\left[-i \omega_{n} t_{1} /\left(2\left|\omega_{n}\right| \hat{\iota}_{\mathbf{k}}\right)\right]$, where $i_{\mathbf{k}}^{-1} \equiv\left(1 / t_{1} \tau_{\mathbf{k}}\right)=$ $2 \pi N_{j} t_{1}^{-1} \rho_{0} \sum_{k^{\prime}}\left|u\left(\mathbf{k}-\mathbf{k}^{\prime}\right)\right|^{2}$. Note that $i_{\mathbf{k}}$, which corresponds to the quasiparticle lifetime (QPLT), is expressed as a dimension-less quantity. We model 

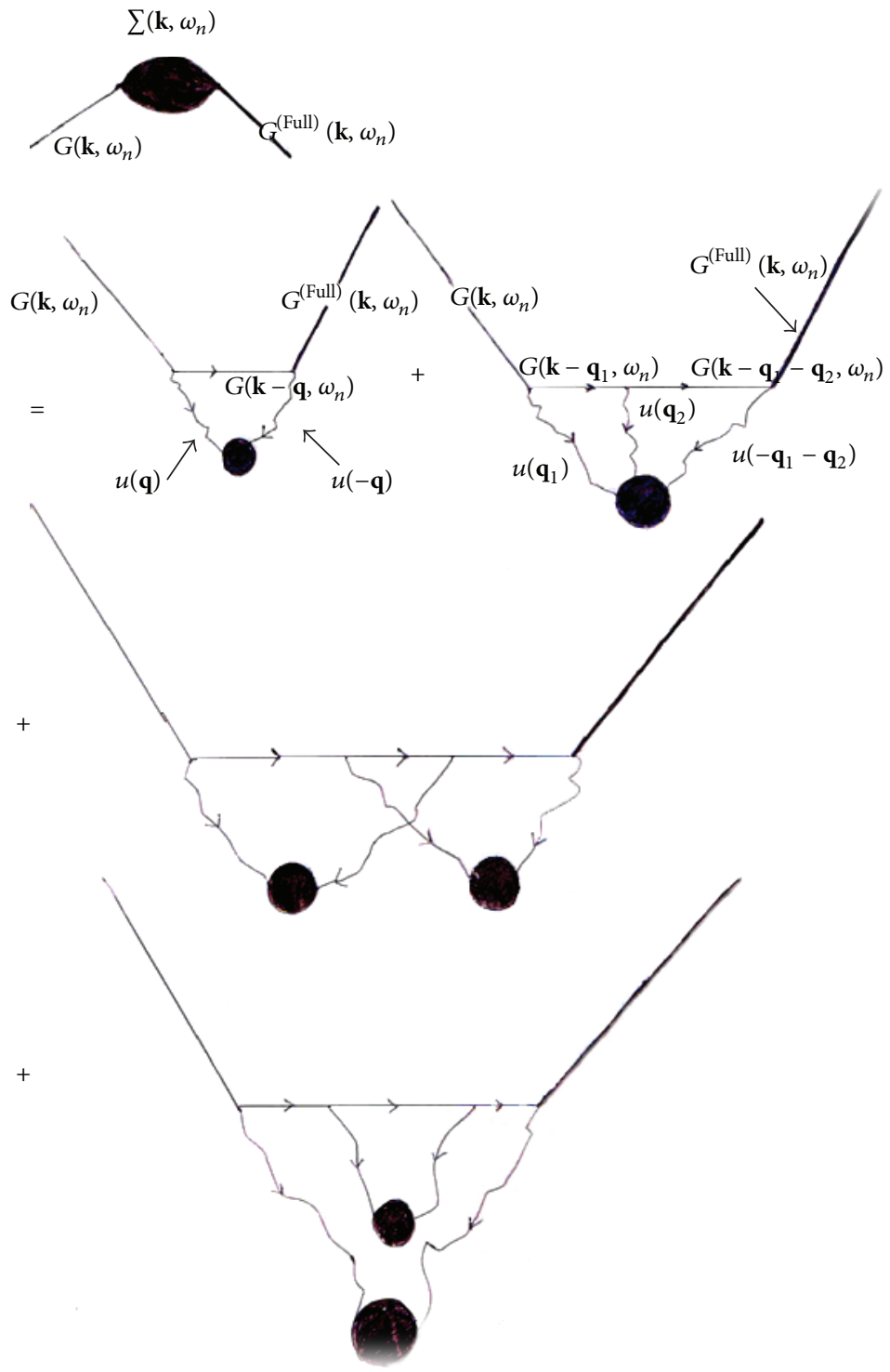

FIGURE 6: A few diagrams contributing to the self-energy. The jagged lines carry momentum but no energy. The total momentum entering each impurity vertex, depicted by a dark circle, is zero. We have assumed that impurities are alike, and distributed randomly. Whereas the first two figures correspond to one impurity vertex, the remaining two correspond to the product of four impurity potentials with nonzero averages. These are the cases where two impurities each give rise to two potentials. Thus, the remaining two figures involve the interference of the scattering by more than one impurity. We have assumed low concentration of impurities, and therefore the figures yield smaller contributions compared to those corresponding to the first two and the other diagrams of the same class involving only one impurity vertex.

$u\left(\left|\mathbf{k}-\mathbf{k}^{\prime}\right|\right)$ by a screened exponential falloff of the form $u\left(\left|\mathbf{k}-\mathbf{k}^{\prime}\right|\right)=\left[\left|u_{0}\right|^{2} \kappa^{2} /\left\{\left|\mathbf{k}-\mathbf{k}^{\prime}\right|^{2}+\kappa^{2}\right\}\right]^{1 / 2}$ to consider the effect of the in-plane impurities, where $\kappa^{-1}$ characterizes the range of the impurity potential. The limit $\kappa \gg\left|\mathbf{k}-\mathbf{k}^{\prime}\right|$, which corresponds to a point-like isotropic scattering potential characterizing the in-plane impurities, will only be considered here for simplicity. For low concentration of impurities and weak disorder potential $\left(t_{1}^{-1} \rho_{0}\left|u_{0}\right|^{2} \ll 1\right)$, we have $\hat{l}_{\mathbf{k}}^{-1} \ll 1$. Upon using the Dyson's equation, under this condition, the full propagator may be written as

$$
\begin{aligned}
G^{(\text {Full })}\left(\mathbf{k}, \omega_{n}\right) \approx & u_{r, \mathbf{k}}^{(1)^{2}}\left[i \omega_{n}-\hat{\varepsilon}_{r, \mathbf{k}}^{(j=+1)}+i\left(\frac{t_{1}}{4 \hat{\iota}_{\mathbf{k}}^{(j=+1)}}\right)\right]^{-1} \\
& +u_{r, \mathbf{k}}^{(2)^{2}}\left[i \omega_{n}-\hat{\varepsilon}_{r, \mathbf{k}}^{(j=-1)}+i\left(\frac{t_{1}}{4 \hat{\imath}_{\mathbf{k}}^{(j=-1)}}\right)\right]^{-1},
\end{aligned}
$$


where

$$
\begin{aligned}
\hat{\varepsilon}_{r, \mathbf{k}}^{(j= \pm 1)}=\left(\frac{1}{2}\right)\left\{\xi_{\mathbf{k}}^{\prime}+j R_{\mathbf{k}}^{1 / 2} \cos \left(\frac{\theta_{\mathbf{k}}}{2}\right)\right\}, \quad \xi_{\mathbf{k}}^{\prime}=\varepsilon_{\mathbf{k}}-\mu+\Sigma_{e} \\
R_{\mathbf{k}}=\left[E_{1}(\mathbf{k})^{4}+E_{2}(\mathbf{k})^{4}\right]^{1 / 2}, \\
\tan \left(\theta_{\mathbf{k}}\right)=\pi-\left(\frac{E_{2}(\mathbf{k})^{2}}{E_{1}(\mathbf{k})^{2}}\right), \\
E_{1}(\mathbf{k})^{2}=\left(\xi_{\mathbf{k}+\mathbf{Q}}+2 w_{\mathbf{k}}\right)^{2}-\xi_{\mathbf{k}}^{2}+\xi_{\mathbf{k}}^{\prime 2}-\left(\frac{t_{1}^{2} \mathbf{l}_{\mathbf{k}}}{4}\right) \\
-4 \Sigma_{e}\left(u_{\mathbf{k}}^{2} \varepsilon_{\mathbf{k}}^{(L)}+v_{\mathbf{k}}^{2} \varepsilon_{\mathbf{k}}^{(U)}\right), \\
E_{2}(\mathbf{k})^{2}=\xi_{\mathbf{k}}^{\prime}\left(\frac{t_{1}}{\hat{l}_{\mathbf{k}}}\right)-\left(\frac{t_{1} \hat{l}_{\mathbf{k}}^{-1}}{2}\right)\left(u_{\mathbf{k}}^{2} \varepsilon_{\mathbf{k}}^{(L)}+v_{\mathbf{k}}^{2} \varepsilon_{\mathbf{k}}^{(U)}\right), \\
u_{r, \mathbf{k}}^{(1)^{2}}=\left[\hat{\varepsilon}_{r, \mathbf{k}}^{(j=+1)}-\left(u_{\mathbf{k}}^{2} \varepsilon_{\mathbf{k}}^{(L)}+v_{\mathbf{k}}^{2} \varepsilon_{\mathbf{k}}^{(U)}\right)\right] \\
\times\left[\hat{\varepsilon}_{r, \mathbf{k}}^{(j=+1)}-\hat{\varepsilon}_{r, \mathbf{k}}^{(j=-1)}\right]^{-1}, \\
u_{r, \mathbf{k}}^{(2)}=\left[\left(u_{\mathbf{k}}^{2} \varepsilon_{\mathbf{k}}^{(L)}+v_{\mathbf{k}}^{2} \varepsilon_{\mathbf{k}}^{(U)}\right)-\hat{\varepsilon}_{r, \mathbf{k}}^{(j=-1)}\right] \\
\times\left[\hat{\varepsilon}_{r, \mathbf{k}}^{(j=+1)}-\hat{\varepsilon}_{r, \mathbf{k}}^{(j=-1)}\right]^{-1} .
\end{aligned}
$$

The QPLT for $j= \pm 1$ are given by

$$
\frac{1}{\hat{\imath}_{\mathbf{k}}^{(j= \pm 1)}}=\left\{\hat{\imath}_{\mathbf{k}}^{-1}+j 2 t_{1}^{-1} R_{\mathbf{k}}^{1 / 2} \sin \left(\frac{\theta_{\mathbf{k}}}{2}\right)\right\} .
$$

We have calculated formal expressions of the propagator $G^{(\text {Full })}\left(\mathbf{k}, \omega_{n}\right)$ and reciprocal QPLT $\left(1 / i_{\mathbf{k}}^{(j= \pm 1)}\right)$ above with the inclusion of impurity scattering. The corresponding retarded Green's function $G^{(R)}(\mathbf{k}, t)$, in units such that $\hbar=1$, is given by $G^{(R)}(\mathbf{k}, t)=$ $\int_{-\infty}^{+\infty}(d \omega / 2 \pi) \exp (-i \omega t) G^{(\text {Full })}(\mathbf{k}, \omega)$ where in the upper half-plane $G^{(\text {Full) }}\left(\mathbf{k}, \omega_{n}\right)$ is given by (28) while in the lower half-plane

$$
\begin{aligned}
G^{(\text {Full })}\left(\mathbf{k}, \omega_{n}\right) \approx & u_{r, \mathbf{k}}^{(1)^{2}}\left[i \omega_{n}-\hat{\varepsilon}_{r, \mathbf{k}}^{(j=+1)}-i\left(\frac{1}{4 \beta \iota_{\mathbf{k}}^{(j=+1)}}\right)\right]^{-1} \\
& +u_{r, \mathbf{k}}^{(2)}\left[i \omega_{n}-\hat{\varepsilon}_{r, \mathbf{k}}^{(j=-1)}-i\left(\frac{1}{4 \beta \iota_{\mathbf{k}}^{(j=-1)}}\right)\right]^{-1} .
\end{aligned}
$$

Thus, $G^{(R)}\left(\mathbf{k}, \omega^{\prime}\right)=\int_{-\infty}^{+\infty} d t \exp \left(i \omega^{\prime} t\right) G^{(R)}(\mathbf{k}, t)$ is given by (31) with $\omega$ real. We obtain

$$
G^{(R)}(\mathbf{k}, t)=\sum_{v=1,2, j=( \pm 1)} u_{r, \mathbf{k}}^{(v)^{2}} i \exp \left(-i \hat{\varepsilon}_{r, \mathbf{k}}^{(j)} t-\left(\frac{t}{4 \beta i_{\mathbf{k}}^{(j)}}\right)\right) \theta(t),
$$

where for $v=1$ and $v=2$, respectively, are the values of the index $v$ which have correspondence with the index $j$ equal to +1 and -1 . The unit step function $\theta(t)=\int_{-\infty}^{+\infty}(i d \omega / 2 \pi)\left\{\exp (-i \omega t) /\left(\omega+i 0^{+}\right)\right\}$. The electronic excitations in cuprates are thus demonstrably particle-hole mixed quasiparticles in the pseudogap phase with finite lifetime for the states of definite momentum due to the impurity scattering. Using the integral representation of $\theta(t)$ above, it is not difficult to show that

$$
\begin{aligned}
G^{(R)}\left(k, \omega^{\prime}\right)= & \int_{-\infty}^{+\infty} d t \exp \left(i \omega^{\prime} t\right) G^{(R)}(k, t) \\
= & \sum_{v=1,2 ; j=( \pm 1)} u_{r, \mathbf{k}}^{(v)^{2}}\left[\omega^{\prime}-\hat{\varepsilon}_{r, \mathbf{k}}^{(j)}+\left(\frac{i t_{1}}{4 \iota_{\mathbf{k}}^{(j)}}\right)\right] \\
& \times\left[\left(\omega^{\prime}-\hat{\varepsilon}_{r, \mathbf{k}}^{(j)}\right)^{2}+\left(\frac{t_{1}}{4 \hat{\iota}_{\mathbf{k}}^{(j)}}\right)^{2}\right]^{-1} .
\end{aligned}
$$

The dimensionless density of states $\rho(\mathbf{k}, \omega) \equiv$ $\left(-1 / 2 \pi^{2} \rho_{0}\right) \operatorname{Im} G^{(R)}(\mathbf{k}, \omega)$, consequently, may be written as $\rho(\mathbf{k}, \omega)=\sum_{v=1,2 ; j=( \pm 1)} \rho^{(j, v)}(\mathbf{k}, \omega)$, where

$$
\rho^{(j, v)}(\mathbf{k}, \omega)=Z_{\mathbf{k}}^{(v)} \gamma_{\mathbf{k}}^{(j)} \times\left[\left(\omega-\hat{\varepsilon}_{r, \mathbf{k}}^{(j)}\right)^{2}+\gamma_{\mathbf{k}}^{(j) 2}\right]^{-1},
$$

$Z_{\mathbf{k}}^{(v)}=\left(1 / 2 \pi^{2} \rho_{0}\right) u_{r, \mathbf{k}}^{(v)}$, and $\gamma_{\mathbf{k}}^{(j)}=\left(t_{1} / 4 \iota_{\mathbf{k}}^{(j)}\right)$ (the level broadening factors). In order to determine the Fermi energy density of states (DOS) $\rho_{\text {Fermi }}(\mathbf{k})$, we shall put $\omega=0$ in (34). It is clear that

$$
\rho_{\text {Fermi }}(\mathbf{k})=\sum_{\nu=1,2 ; j=( \pm 1)} Z_{\mathbf{k}}^{(v)} \gamma_{\mathbf{k}}^{(j)} \times\left[\hat{\varepsilon}_{r, \mathbf{k}}^{(j) 2}+\gamma_{\mathbf{k}}^{(j) 2}\right]^{-1} .
$$

At this stage, assuming low concentration of impurities, one may include the contributions of all such diagrams which involve only one impurity vertex. This gives the equation to determine the total self-energy $\Sigma\left(\mathbf{k}, \omega_{n}\right): \Sigma\left(\mathbf{k}, \omega_{n}\right)=$ $N_{j} \sum_{\mathbf{q}} u(\mathbf{q}) G^{(\text {Full })}\left(\mathbf{k}-\mathbf{q}, \omega_{n}\right) \Gamma\left(\mathbf{k}, \mathbf{q}, \omega_{n}\right)$, where the LippmannSchwinger equation to determine $\Gamma\left(\mathbf{k}, \mathbf{q}, \omega_{n}\right)$ is $\Gamma\left(\mathbf{k}, \mathbf{q}, \omega_{n}\right)=$ $u(-\mathbf{q})+\sum_{\mathbf{q}^{\prime}} u\left(\mathbf{q}^{\prime}-\mathbf{q}\right) G^{(\text {Full })}\left(\mathbf{k}-\mathbf{q}^{\prime}, \omega_{n}\right) \Gamma\left(\mathbf{k}, \mathbf{q}^{\prime}, \omega_{n}\right)$. This is the $t$-martix approximation. Upon using the optical theorem for the $t$-matrix [53], one may write $\Sigma\left(\mathbf{k}, \omega_{n}\right)=i \operatorname{Im} \Gamma\left(\mathbf{k}, \mathbf{k}, \omega_{n}\right)=$ $-i \omega_{n} t_{1} /\left(2\left|\omega_{n}\right| \hat{\Gamma}_{\mathbf{k}}\right)$ where $\hat{\Gamma}_{\mathbf{k}}^{-1}=2 \pi N_{j} t_{1}^{-1} \rho_{0} \sum_{\mathbf{k}^{\prime}}\left|\Gamma\left(\mathbf{k}, \mathbf{k}^{\prime}\right)\right|^{2}$. Thus, the effect of the inclusion of contribution of all the above-mentioned diagrams, which involve only one impurity vertex, is to replace the Born approximation for scattering by the exact scattering cross-section for a single impurity, that is, $\stackrel{\imath}{\mathbf{k}}^{-1} \rightarrow \tilde{\Gamma}_{\mathbf{k}}^{-1}$. 
Since $G\left(\mathbf{k}, \omega_{n}\right)$ and $u(\mathbf{q})$ are known, one can determine $\Gamma_{\mathbf{k}}^{-1}$ in terms of $u(\mathbf{k})$. In the limit $\kappa \gg\left|\mathbf{k}-\mathbf{k}^{\prime}\right|$, the disorder potential $u(|\mathbf{q}|) \approx\left|u_{0}\right|$, and, therefore, we obtain $\Gamma\left(\mathbf{k}, \omega_{n}\right) \approx$ $\left|u_{0}\right| /\left(1-\left|u_{0}\right| G\left(\mathbf{k}, \omega_{n}\right)\right)$. In view of $G\left(\mathbf{k}, \omega_{n}\right)$ given above (27), we find that $\operatorname{Im} \Gamma\left(\mathbf{k}, \omega_{n}\right) \approx-\rho_{0} \pi\left|u_{0}\right|^{2} /\left(1+\rho_{0}^{2} \pi^{2}\left|u_{0}\right|^{2}\right)$. From the equation $\left(i \operatorname{Im} \Gamma\left(\mathbf{k}, \mathbf{k}, \omega_{n}\right)\right)=-i \omega_{n} t_{1} /\left(2\left|\omega_{n}\right| \dot{\Gamma}_{\mathbf{k}}\right)$, we consequently find that $\Gamma_{\mathbf{k}}^{-1}$, in the first approximation, is given by $\Gamma_{0}^{-1}=\left[2 \pi N_{j} t_{1}^{-1} \rho_{0}\left|u_{0}\right|^{2} /\left(1+\rho_{0}^{2} \pi^{2}\left|u_{0}\right|^{2}\right)\right]$. We now replace $i_{\mathbf{k}}^{-1}$ by $\hat{\Gamma}_{0}^{-1}$ in (30) and (35). The reciprocal QPLT for the electron and the hole quasiparticles are plotted in Figure 7 as a function of $\hat{\Gamma}_{0}^{-1}$ (measure of disorder potential) for $\Sigma_{e} / t_{1}=$ -1.2 . The former is assumed to be centered at $( \pm \pi, 0)$ and $(0, \pm \pi)$ whereas the latter at $( \pm \pi / 2, \pm \pi / 2)$ of the first Brillouin zone. We find that the former is short-lived compared to the latter for very weak to moderately weak disorder potential. We note that, even though $\Gamma_{\mathbf{k}}$ is found to be $\mathbf{k}$-independent in the first approximation, the term $\pm 2 t_{1}^{-1} R_{\mathbf{k}}^{1 / 2} \sin \left(\theta_{\mathbf{k}} / 2\right)$ in (30) will ensure that $1 / \hat{\Gamma}_{\mathbf{k}}^{(j= \pm 1)}=\left\{\hat{\Gamma}_{0}^{-1}+j 2 t_{1}^{-1} R_{\mathbf{k}}^{1 / 2} \sin \left(\theta_{\mathbf{k}} / 2\right)\right\}$ are momentum-dependent. In Figure 8, we have shown the contour plots of the Fermi energy DOS or spectral density given by (35) on the Brillouin zone (BZ) at $9.94 \%$ hole doping where $\Sigma_{e} / t_{1}(=-1.2)$ redefines the chemical potential. Whereas for Figure 8(a) the disorder potential measure $\Gamma_{0}^{-1}=$ 0.05 , for Figure $8(\mathrm{~b})$ it is 0.30 . The usual Fermi arc (Figure $8(\mathrm{a}))$ for very low value of $\hat{\Gamma}_{0}^{-1}\left(\hat{\Gamma}_{0}^{-1} \ll 1\right)=0.05$ gets nearly washed out (Figure $8(\mathrm{~b})$ ) for higher value $\Gamma_{0}^{-1}=0.3$ since, as seen in Figure 7, the increase in $\Gamma_{0}^{-1}$ makes the lifetime of nodal excitations shorter. Thus, the disorder seems to have significant influence on the Fermi energy DOS.

3.3. Entropy and Specific Heat. The dimensionless entropy per unit cell is given by $S=\beta^{2}(\partial \Omega / \partial \beta)$. For the pseudogapped (PG) phase $\left(T<T^{*}\right)$ and the normal phase $\left(T>T^{*}\right)$, the entropy expressions are $S_{\mathrm{PG}}=2 \int d(\mathbf{k} a) \rho_{\text {Fermi }}(\mathbf{k}) s_{\mathrm{PG}}(\mathbf{k})$ and $S_{N}=2 \int d(\mathbf{k} a) \rho_{\text {Fermi }}(\mathbf{k}) s_{N}(\mathbf{k})$, respectively, where

$$
\begin{aligned}
& s_{\mathrm{PG}}(\mathbf{k})=\sum_{j(=U, L)}[ \ln \left(\exp \left(-\beta \varepsilon_{\mathbf{k}}^{(j)}\right)+1\right) \\
&+\left(\beta \varepsilon_{\mathbf{k}}^{(j)}+\beta^{2}\left(\frac{\partial \varepsilon_{\mathbf{k}}^{(j)}}{\partial \beta}\right)\right) \\
&\left.\times\left(\exp \left(\beta \varepsilon_{\mathbf{k}}^{(j)}\right)+1\right)^{-1}\right], \\
& s_{N}(\mathbf{k})=\sum_{j=1,2}[\ln (\left.\exp \left(-\beta \varepsilon_{j}(\mathbf{k})\right)+1\right) \\
&+\left(\beta \varepsilon_{j}(\mathbf{k})+\beta^{2}\left(\frac{\partial \varepsilon_{j}(\mathbf{k})}{\partial \beta}\right)\right) \\
& \times\left(\exp \left(\beta \varepsilon_{j}(\mathbf{k})+1\right)^{-1}\right],
\end{aligned}
$$

$\varepsilon_{1}(\mathbf{k})=\varepsilon_{\mathbf{k}}$, and $\varepsilon_{2}(\mathbf{k})=\varepsilon_{\mathbf{k}+\mathbf{Q}}$. We calculate the value of the entropy difference between the DDW state and the normal state $\left(\Delta S=S_{\mathrm{PG}}\left(T<T^{*}\right)-S_{N}\left(T>T^{*}\right)\right)$. The specific heat

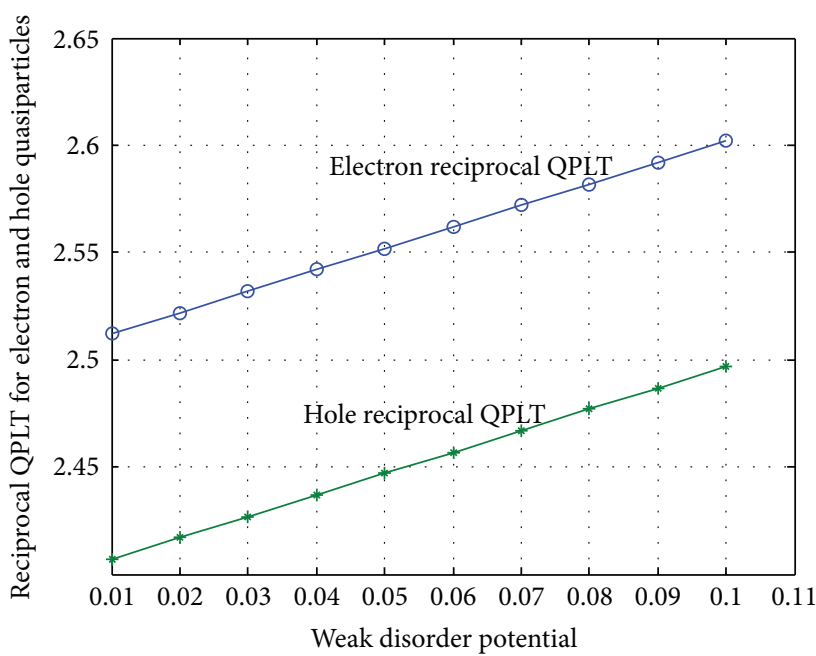

FIGURE 7: A plot of the electron and hole reciprocal QPLT as a function of weak disorder potential using (30) for $\Sigma_{e} / t_{1}=-1.2$. The former is assumed to be centered at $( \pm \pi, 0)$ and $(0, \pm \pi)$ whereas the latter at $( \pm \pi / 2, \pm \pi / 2)$ of the first Brillouin zone. One notices that the former is short-lived compared to the latter for very weak to moderately weak disorder potential.

$\left(C_{\mathrm{el}}=-\beta(\partial S / \partial \beta)\right)$, for the pseudo-gapped $(\mathrm{PG})$ phase $(T<$ $\left.T^{*}\right)$, is

$$
\begin{aligned}
C_{\mathrm{el}} \approx 2 k_{B} \int d(\mathbf{k} a) \rho_{\text {Fermi }}(\mathbf{k}) \sum_{j(=U, L)}\left(\beta \varepsilon_{\mathbf{k}}^{(j)}\right)^{2} \times \exp \left(\beta \varepsilon_{\mathbf{k}}^{(j)}\right) \\
\times\left(\exp \left(\beta \varepsilon_{\mathbf{k}}^{(j)}\right)+1\right)^{-2} .
\end{aligned}
$$

We have ignored the temperature dependence of the chemical potential above. For the $\mathbf{k}$-summation purpose, we shall first divide the BZ into finite number of rectangular patches. We shall next determine the numerical values corresponding to each of these patches of the momentum-dependent density (e.g., for $C_{\mathrm{el}}$ the momentum dependent density is $\rho_{\text {Fermi }}(\mathbf{k}) \sum_{j(=U, L)}\left(\beta \varepsilon_{\mathbf{k}}^{(j)}\right)^{2} \times \exp \left(\beta \varepsilon_{\mathbf{k}}^{(j)}\right) \times$ $\left.\left(\exp \left(\beta \varepsilon_{\mathrm{k}}^{(j)}\right)+1\right)^{-2}\right)$ and sum these values. We generate these values through the surface plots using "Matlab." With these inputs, we now embark on a calculation of the entropy and the specific heat. The entropy difference $\Delta S\left(=S_{\mathrm{PG}}-S_{\text {Normal }}\right)$ between the PG and normal paramagnetic states is negative (see Table 1). As an ordered state is expected to have lower entropy, there is nothing unusual about it.

The physical quantities, such as specific heat $C_{\mathrm{el}}$, electrical resistivity $\rho$, and magnetic susceptibility $\chi$, are expected to show anomalous temperature dependences due to the onset of exotic DDW ordering which, as we have seen, has its origin in the nonnesting property of the dispersion. In order to examine this aspect, we have calculated the specific heat, using (38), as a function of decreasing temperature in the PG phase starting from $T^{*} \approx 200 \mathrm{~K}$ at a fixed underdoping level $\approx 9.94 \%$. The dimensionless electronic specific heat displays a non-Fermi liquid behavior with decreasing temperature in 


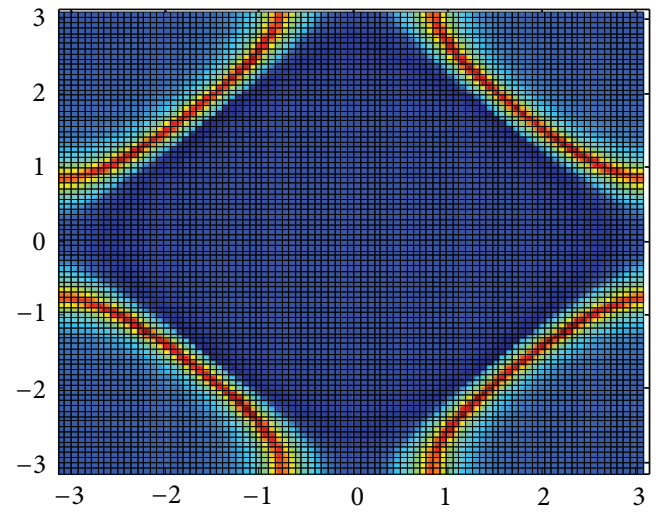

(a)

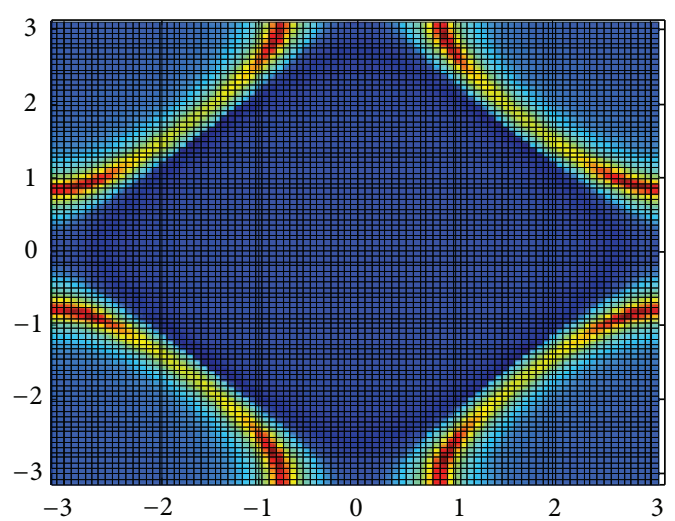

(b)

Figure 8: The contour plots of the Fermi energy DOS on the Brillouin zone (BZ) at 9.94\% hole doping for $\Sigma_{e} / t_{1}=-1.2$ which redefines the chemical potential. The scale of the plots is from -0.2 to 0.6 . In (a) $\Gamma_{0}^{-1}=0.05$ whereas in (b) it is 0.30 . The usual Fermi arc scenario in (a) gets nearly washed out in (b) due to increase in the disorder potential.

TABLE 1: The values of the pseudo-gap (PG) state entropy $S_{\mathrm{PG}}$ given by (36) (column (ii)) and the normal state entropy $S_{N}$ given by (37) (column (iv)) at the temperatures indicated at the doping level $9.94 \%$. We also have noted the values of $C_{\mathrm{el}}$ calculated using (38). We identify the pseudo-gap temperature $T^{*}(\sim 200 \mathrm{~K})$ as the one at which the entropy difference $\Delta S=S_{\mathrm{PG}}-S_{N}$ becomes zero. The entropy is almost a smoothly increasing function of temperature. There appears to be a small discontinuity (not clearly discernable) in entropy at $T^{*}$ during the passage from the PG state to normal state. We find that there is no discontinuity in the electronic specific heat $C_{\mathrm{el}}$ either.

\begin{tabular}{|c|c|c|c|}
\hline (i) & (ii) & (iii) & (iv) \\
\hline$T($ in $\mathrm{K})$ & $\begin{array}{l}\text { Entropy and } \\
\text { specific heat }\end{array}$ & $T($ in $\mathrm{K})$ & $\begin{array}{l}\text { Entropy and } \\
\text { specific heat }\end{array}$ \\
\hline 100 & $348.4303 ; 0.0065$ & 200 & $779.3915 ; 0.0342$ \\
\hline 120 & $418.3876 ; 0.0100$ & 210 & $824.0177 ; 0.0360$ \\
\hline 135 & $471.2098 ; 0.0129$ & 220 & $869.3290 ; 0.0378$ \\
\hline 136 & $471.2035 ; 0.0130$ & 230 & $915.0129 ; 0.0396$ \\
\hline 143 & $499.5318 ; 0.0144$ & 240 & $961.5105 ; 0.0413$ \\
\hline 147 & $512.7416 ; 0.0151$ & 250 & $1008.8000 ; 0.0429$ \\
\hline 160 & $567.3138 ; 0.0168$ & & \\
\hline 175 & $624.8505 ; 0.0245$ & & \\
\hline 200 & $777.1552 ; 0.0341$ & & \\
\hline
\end{tabular}

Figure 9(b): $C_{\mathrm{el}} \approx 3.1218-0.9001\left[\left(k_{B} T / t_{1}\right) \log \left(k_{B} T / t_{1}\right)\right]+$ $0.0713\left[\left(k_{B} T / t_{1}\right) \log \left(k_{B} T / t_{1}\right)\right]^{2}$. The plot above shows that the second-degree polynomial fit (in $\left[\left(k_{B} T / t_{1}\right) \log \left(k_{B} T / t_{1}\right)\right]$ ) approximately takes care of the anomalous behavior in $C_{\mathrm{el}}$. In the normal phase, $C_{\mathrm{el}}$ is linear in $T$ as shown in Figure 9(c). The Sommerfield constant $\gamma$ is found to be $1.7143 \times 10^{-4} k_{B}$. We find that, for $\left(T-T^{*}\right) / T^{*} \rightarrow 0_{ \pm}, C_{\mathrm{el}} \approx 1.7100 \times 10^{-4} k_{B} T^{*}$, that is, there is no discontinuity in $C_{\mathrm{el}}$ at the PG temperature $T^{*}$. Within a mean field theoretic framework, we observe that even slightly shorter lifetime of electron quasiparticles for a weak disorder potential $\sim 0.1$ (see Figure 7 ) in PG phase leads to a severe non-Fermi liquid (NFL) behavior in specific heat which is absent in the normal phase.
3.4. Transport Properties. We now consider the simplest description of quasiparticle transport [54] in the DDW state in terms of the Boltzmann equation in the relaxationtime approximation. The elastic impurity scattering with the inclusion of the disorder potential leads to a momentumdependent relaxation time as seen in the Section 3.2. In our calculations and graphical representations in Section 2 , however, we assumed an intrinsic lifetime broadening $\left(\gamma / t_{1}\right) \sim 0.1$ independent of momentum. We make the same assumption here in the first approximation and consider a momentum- and energy-independent relaxation time $\tau$ and its dimensionless counterpart $\tau_{0}=t_{1} \tau / \hbar$. This is seemingly quite adequate for elastic impurity scattering for a weak disorder potential $\sim 0.1$ as the lifetimes of electron quasiparticles are not strikingly different from that of hole quasiparticles (see Figure 7). The electrical and thermal conductivities may then be expressed as

$$
\begin{aligned}
& \sigma_{x x}=-\left(\frac{2 e^{2}}{h}\right) \tau_{0} \sum_{\mathbf{k}, j(=U, L)}\left(\frac{\partial f_{\mathbf{k}}^{(j)}}{\partial \varepsilon_{\mathbf{k}}^{(j)}}\right)\left(v_{k, x}^{(j)}\right)^{2}, \\
& \sigma_{x y}=-\left(\frac{D e^{2}}{h}\right) \tau_{0}^{2} \sum_{\mathbf{k}, j=U, L)}\left(\frac{\partial f_{\mathbf{k}}^{(j)}}{\partial \varepsilon_{\mathbf{k}}^{(j)}}\right)\left(v_{k, x}^{(j)}\right) \\
& \times\left[v_{k, y}^{(j)}\left(\frac{\partial v_{k, y}^{(j)}}{\partial k_{x} a}\right)-v_{k, x}^{(j)}\left(\frac{\partial v_{k, y}^{(j)}}{\partial k_{y} a}\right)\right], \\
& \alpha_{x x}=\left(\frac{2 e^{2}}{h}\right)\left(\frac{\tau_{0}}{e T}\right) \sum_{\mathbf{k}, j=U, L)} \varepsilon_{\mathbf{k}}^{(j)}\left(\frac{\partial f_{\mathbf{k}}^{(j)}}{\partial \varepsilon_{\mathbf{k}}^{(j)}}\right)\left(v_{k, x}^{(j)}\right)^{2}, \\
& \alpha_{x y}=\left(\frac{D e^{2}}{h e T}\right) \tau_{0}^{2} \sum_{\mathbf{k}, j(=U, L)} \varepsilon_{\mathbf{k}}^{(j)}\left(\frac{\partial f_{\mathbf{k}}^{(j)}}{\partial \varepsilon_{\mathbf{k}}^{(j)}}\right)\left(v_{k, x}^{(j)}\right) \\
& \times {\left[v_{k, y}^{(j)}\left(\frac{\partial v_{k, y}^{(j)}}{\partial k_{x} a}\right)-v_{k, x}^{(j)}\left(\frac{\partial v_{k, y}^{(j)}}{\partial k_{y} a}\right)\right], }
\end{aligned}
$$




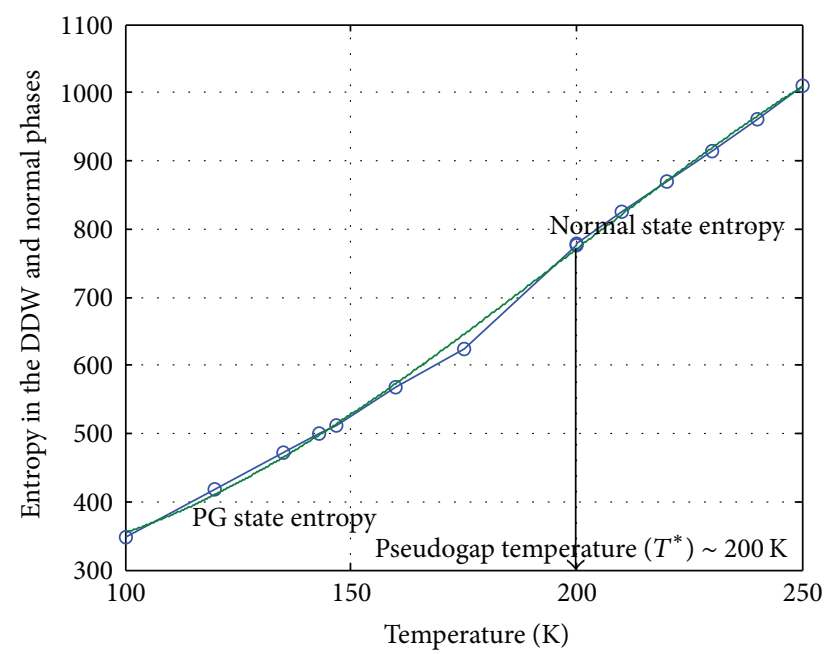

(a)

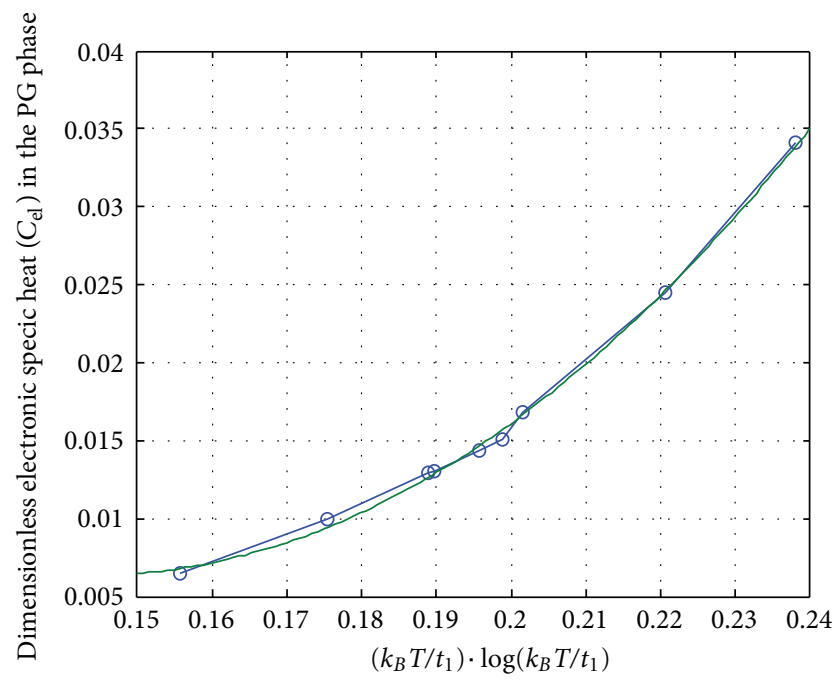

(b)

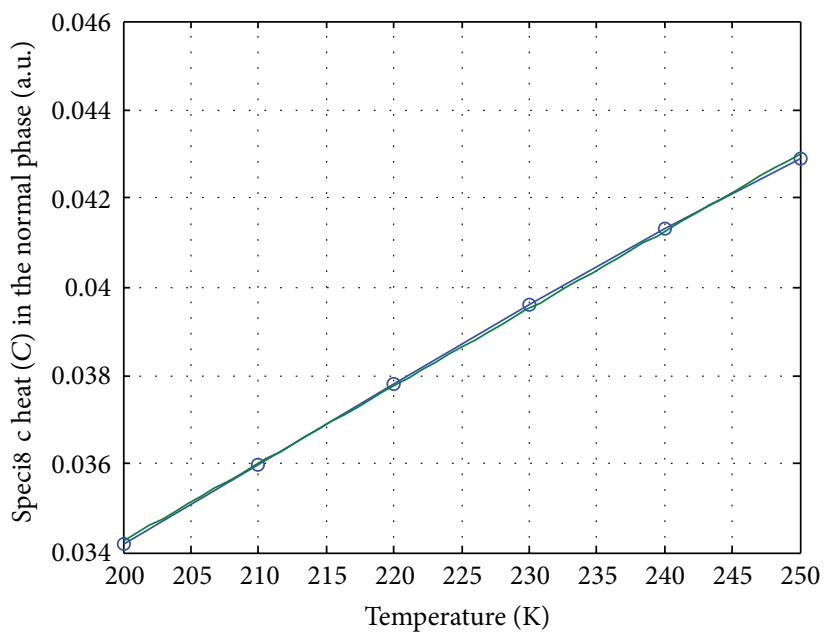

(c)

Figure 9: (a) A plot of the entropy in the DDW and normal phases as a function of temperature. We identify the pseudogap temperature $T^{*}$ $(\sim 200 \mathrm{~K})$ as the one at which the entropy difference $\Delta S=S_{\mathrm{PG}}-S_{N}$ becomes zero. (b) A plot of the dimensionless electronic specific heat in the PG phase as a function of $\left[\left(k_{B} T / t_{1}\right) \log \left(k_{B} T / t_{1}\right)\right]$ and a second-degree polynomial fit. (c) A plot of the specific heat $\left(C_{\mathrm{el}}\right)$ in the normal phase as a function of $T$. We have the usual linear dependence of $C_{\mathrm{el}}$ on $T$ in the normal phase.

where the dimensionless quantity $D \equiv 4 \pi\left(a / l_{B}\right)^{2}(a / L)^{2}$, $l_{B} \equiv(\hbar / e B)^{1 / 2}$ is the magnetic length, $\sum_{j}$ runs over $j=$ $(U, L)$, and $\varepsilon_{\mathbf{k}}^{(j)}, f_{\mathbf{k}}^{(j)}, v_{k, \alpha}^{(j)}(\alpha=x, y)=\left(\partial \varepsilon_{\mathbf{k}}^{(j)} / \partial k_{\alpha} a\right)$ are the corresponding energies, Fermi functions and velocity components, respectively. The energies $\varepsilon_{\mathbf{k}}^{(j)}$ are expressed in units of the first neighbor hopping $t_{1}$. Equation (39) shows that, in the presence of multiple quasiparticle bands, the elements of the conductivity tensors are simply a superposition of the contributions from the individual bands. The reason for the interband contributions not showing up in (39) is that the spectral functions, appearing in the general quantum Boltzmann equations [54], are replaced by $\delta$ functions at the quasiparticle energies. The spectral densities do appear as bunch of $\delta$ functions as can be seen in the discussions below in (9) in Section 2. We have artificially introduced an intrinsic lifetime broadening though to make these $\delta$ functions appear Lorentzians. Figure 10 shows a clear discontinuity in $\alpha_{x x}$ at $T^{*} \sim 200 \mathrm{~K}$. The dimensionless Lorentz number $L \equiv$ $\alpha_{x x} / T \sigma_{x x}\left(\sim 6.7 \times 10^{-4}\right)$ is nearly a constant in the PG phase. The reason for the slight nonconstancy may be ascribed to the total omission of the interband contributions alluded to above. We see that $L \ll 1$ and $\alpha_{x x} \sim 1$, and $\sigma_{x x} \gg 1$ (see Table 2) inside the DDW phase. This shows somewhat metallic character of the DDW phase. It indicates that, though the DDW phase is characterized by the gap maxima in the antinodal regions and the zero gap in the nodal regions of the $\mathrm{BZ}$, the electron quasiparticles which inhabit the former region must have a shorter lifetime compared to those inhabiting the latter region (cf. Figure 7). In fact, 


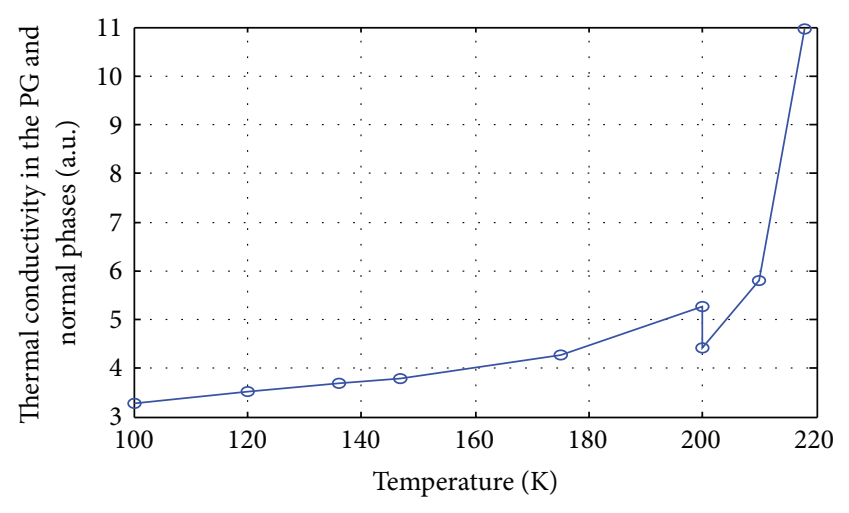

Figure 10: A plot of the values of the pseudogap (PG) state quasiparticle (dimensionless) thermal conductivity $\left(\alpha_{x x}^{\mathrm{PG}}\right)$ and the normal state thermal conductivity $\left(\alpha_{x x}^{N}\right)$ as a function of temperature ( $T$ in Kelvin) at the doping level $9.94 \%$. The discontinuity in $\alpha_{x x}$ is clearly visible at $T^{*} \sim 200 \mathrm{~K}$. The dimensionless Lorentz number ( $L=\alpha_{x x} / T \sigma_{x x}$ ), at the doping level $9.94 \%$ in the PG phase, is found to be of $\sim 6.7 \times 10^{-4}$ (indicating that (dimensionless) $\sigma_{x x} \gg 1$ in the PG phase) albeit with slight variation. The variation may be due to the total omission of the interband contributions in (39).

precisely for this reason the thermal conductivity $\alpha_{x x}$ shows discontinuity in the passage from the PG to normal phase; in PG phase the dominant contributions to $\alpha_{x x}$ come through the states corresponding to Fermi arc including the nodal quasiparticles (see Figure 11(a)) whereas in the normal phase the main contributions are from the portion of the arc close to the antinodal region $( \pm \pi, 0)$ (see Figure 11(b)). As shown in Figure 11(c), the main contributions to $\sigma_{x x}$ as well come through the states corresponding to Fermi arc. Furthermore, we also find that as we move to the SC phase from the PG phase along the line indicated in Figure 1, there is considerable enhancement in $\sigma_{x x}$ as expected.

The underlying assumption in writing down the expressions for the electrical and the thermal Hall conductivities $\left(\sigma_{x y}, \alpha_{x y}\right)$ in (39) is that we are in the linear response regime. The invocation of the Landau level (LL) quantization is not necessary in this regime as the magnetic field $(B)$ may be assumed to be much smaller than 1 Tesla. Besides, the LL quantization is expected to be relevant for a large and ultraclean sample. The normal state dispersion in (10) now appears as

$$
\begin{aligned}
\varepsilon_{\mathbf{k}}(B)= & -2 t_{1}\left(\cos \left(k_{x} a\right)+\cos \left(k_{y} a+\varphi\right)\right) \\
& +4 t_{2} \cos \left(k_{x} a\right) \cos \left(k_{y} a+\frac{\varphi}{2}\right) \\
& -2 t_{3}\left(\cos 2 k_{x} a+\cos 2\left(k_{y} a+\varphi\right)\right)+4 t_{2},
\end{aligned}
$$

where the vector potential $\mathbf{A}$ may be assumed to be in the Landau gauge: $\mathbf{A}=\left(0-B_{x} 0\right)$. The hopping amplitudes $t_{i j}$, corresponding to the sites $\mathbf{i}$ and $\mathbf{j}$, now may assume the form $\left[t_{i j} \exp \left(a_{i j}\right)\right]$, where $a_{i j}=\left(\pi / \Phi_{0}\right) \int_{\mathbf{j}}^{\mathbf{i}} \mathbf{A} \cdot \mathrm{dL}$ and $\Phi_{0}=$ $(h / 2 e)$. For the first neighbor hopping, say, corresponding to the sites $\mathbf{i}=(a, 0)$ and $\mathbf{j}=(a, a)$, the quantity $a_{i j}=$
TABLE 2: The values of the pseudo-gap (PG) state electrical hall conductivity $\left(\sigma_{x y}\right)$, longitudinal conductivity $\left(\sigma_{x x}\right)$, and The Hall angle $\left(\theta_{H}\right)$ for $B=0.08$ Tesla. We find $\tan \theta_{H} \equiv \sigma_{x y} / \sigma_{x x} \sim 0.01$ in the PG phase.

\begin{tabular}{lccc}
\hline (i) & (ii) & (iii) & (iv) \\
$T($ in K) & $\sigma_{x y}$ & $\sigma_{x x}$ & $\tan \theta_{H} \equiv \sigma_{x y} / \sigma_{x x}$ \\
\hline 100 & 0.4216 & 49.1248 & 0.0086 \\
120 & 0.3898 & 43.9357 & 0.0089 \\
135 & 0.4070 & 40.7728 & 0.0100 \\
137 & 0.4029 & 40.5583 & 0.0099 \\
147 & 0.3948 & 38.6188 & 0.0102 \\
175 & 0.4046 & 36.6506 & 0.0110 \\
\hline
\end{tabular}

$-\left(\pi / \Phi_{0}\right) \int_{(a, a)}^{(a, 0)} B x d y=\varphi$, where $\varphi=\left(2 \pi e B a^{2} / h\right)$ is the Peierls phase factor. Similarly, for the second neighbor hopping, say, corresponding to the sites $\mathbf{i}=(a, 0)$ and $\mathbf{j}=$ $(2 a, a)$, the quantity $a_{i j}=-\left(\pi / \Phi_{0}\right) \int_{(2 a, a)}^{(a, 0)} B y d y=\varphi / 2$. These explain the reason behind the appearance of $\varphi$ and $\varphi / 2$, respectively, in the first and the second terms of (40). Likewise, the reason for the appearance of $\varphi$ in the third term of (40) could be explained. Taking lattice constant $=3.8 \AA$, we find that $\varphi=2.1998 \times 10^{-4} B$ where $B$ should be in Tesla. For $B \ll 1$ Tesla, $\varphi \ll 1$. Therefore, a nonzero $\varphi \ll 1$ is not expected to affect the linear dependence of $\left(\sigma_{x y}, \alpha_{x y}\right)$ on $B$, as could be apparently inferred from (26), in any significant way. We indeed find the linear dependence of $\left(\sigma_{x y}, \alpha_{x y}\right)$ on $B$ in the PG phase at a given temperature for the doping level $\sim 10 \%$ (see Figure 12). Now as shown in Figure 13, where we have plotted the electrical and the thermal Hall conductivity densities in the first BZ at $T=127 \mathrm{~K}, p=9.94 \%$, and $B=$ 0.08 Tesla, the main contributions to $\left(\sigma_{x y}, \alpha_{x y}\right)$ arise from the density of states corresponding to those portions of the Fermi arc which are not part of the nodal region inhabited by quasiparticles of longer lifetime; there is no contribution from the antinodal regions centered at $[( \pm \pi, 0),(0, \pm \pi)]$ either. These findings are consistent as long as $B \ll 1$ and the temperature is lower than $T^{*}$. Therefore, these are very much supportive of the experimental findings of Doiron-Leyraud et al. [55-57] who have detected quantum oscillations in the electrical resistance of underdoped YBCO establishing the existence of a well-defined Fermi surface (FS) with Fermi pockets in the antinodal region when the superconductivity is suppressed by a strong magnetic field. Furthermore, Riggs et al. [58] have observed the oscillations in the specific heat of YBCO-Ortho II samples (in the presence of a magnetic field $B=45 \mathrm{~T}$ ), the same type of YBCO samples investigated by Doiron-Leyraud and coworkers [55-57], confirming the findings regarding Fermi pockets.

As shown in Table 2, we could find reasonably conducting character of the PG state for the near-nested Fermi surface. We obtain nearly constant small Hall (electrical) conductivity for all the temperatures considered close to $T_{c}$ and those below it in the underdoped region. The longitudinal conductivity $\sigma_{x x}$ varies slowly, while the Hall angle $\tan \theta_{H} \equiv$ $\sigma_{x y} / \sigma_{x x} \ll 1$. 


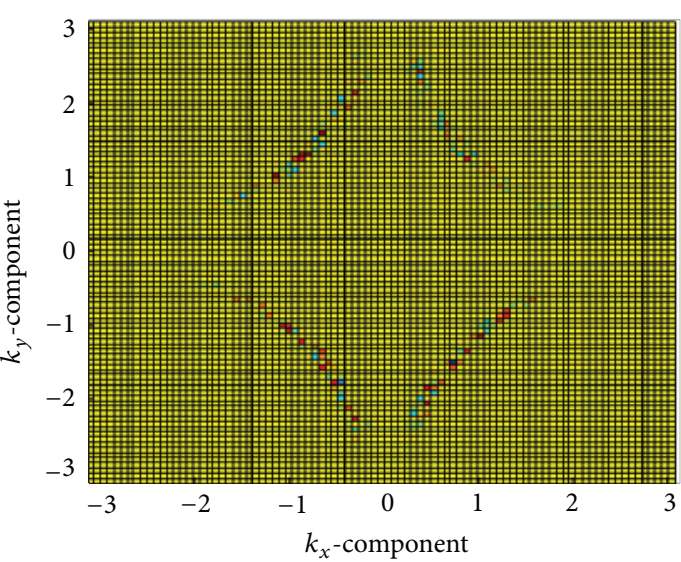

(a)

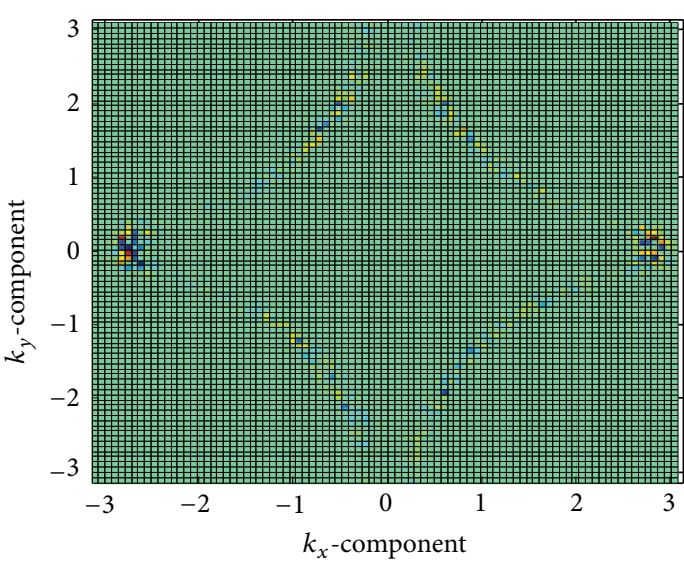

(b)

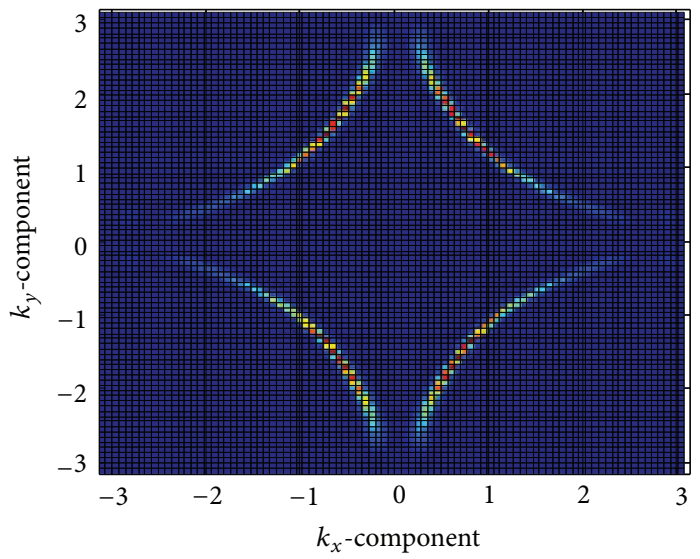

(c)

FIGURE 11: The contour plots of (a) the pseudogap (PG) state quasiparticle (dimensionless) thermal conductivity $\left(\alpha_{x x}^{\mathrm{PG}}\right)$ density, (b) the normal state thermal conductivity $\left(\alpha_{x x}^{N}\right)$ density, and (c) the electrical conductivity $\left(\sigma_{x x}^{\mathrm{PG}}\right)$ density on the first BZ at $120 \mathrm{~K}$. The relative scale of the plots in (a) and (b) is from -3 to +2 while in (c) is from 0 to 0.8 . The dominant contribution to $\left(\alpha_{x x}^{\mathrm{PG}}, \sigma_{x x}^{\mathrm{PG}}\right)$ come from the states corresponding to the Fermi arc. There is, however, no contribution from the antinodal quasiparticles. In the normal phase, the main contributions to $\alpha_{x x}$ are from the portion of the arc close to the antinodal region $( \pm \pi, 0)$.

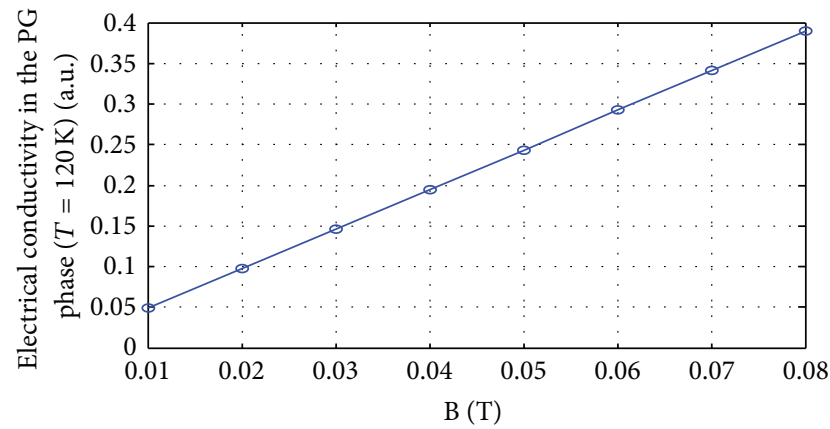

FigURE 12: A 2D plot showing the linear dependence of $\sigma_{x y}$ on $B$ in the PG phase at the temperature $120 \mathrm{~K}$ for the doping level $\sim 10 \%$.

\section{Spectral Weight}

We now focus our attention on the optical spectral weight $[32-40] \operatorname{SW}(T)=\int_{0}^{\infty} \operatorname{Re} \sigma_{x x}(\omega, T)(d \omega / \pi) \propto\left\langle-H_{\text {kin }}\right\rangle$, where $\operatorname{Re} \sigma_{x x}(\omega, T)$ is the real part of the optical conductivity, assuming the direction $x$ to be the direction of the current flow when induced by an electromagnetic field in the same direction. The quantity SW $(T)$ provides valuable information on the evolution of the electronic dynamics with temperature [35]. The issue of the constancy and the temperature dependence of the total optical sum above is discussed at length in [34-37]. One may refer to the works of Baeriswyl et al. [36, 37], in particular, to examine the validity of the relation for $\mathrm{SW}(T)$ above for discrete lattice models at finite temperature. We shall see, for our single-band system of cuprates, that the optical sum is dependent on the temperature when the full temperature dependence of the single-particle distribution due to the tight-binding band dispersion with the van Hove singularity and the unconventional orderings (DDW and DSC) are consistently accounted for. Our treatment produces a similar temperature dependence in this sum as seen in experiments reported nearly a decade ago $[38,39]$. It may be noted that one sometimes introduces a suitable upper cutoff frequency $\dot{\omega}$ in place of $\infty$ in the optical sum above 


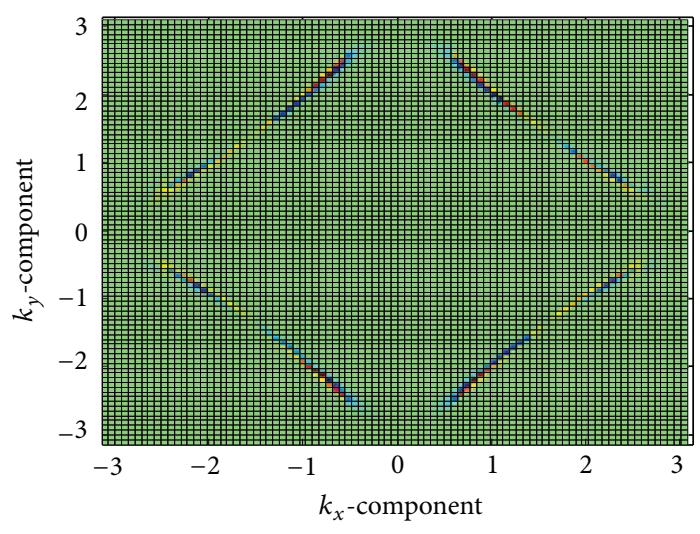

(a)

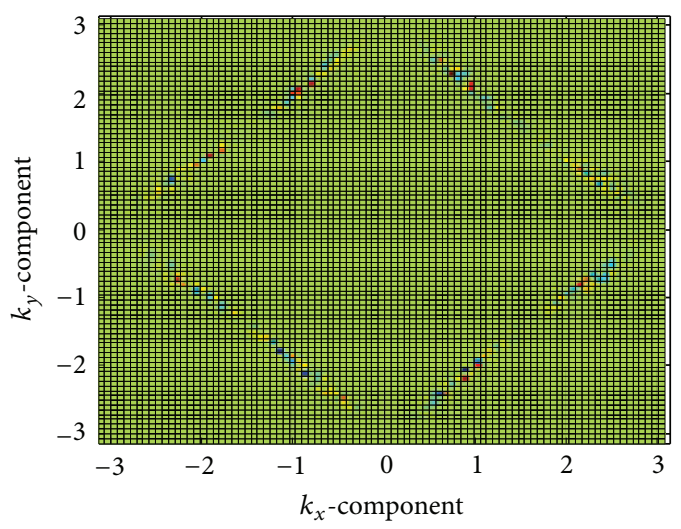

(b)

Figure 13: The contour plots of (a) the electrical Hall conductivity density $\left(\sigma_{x y}^{(\mathrm{PG})}(\mathbf{k}, T, B)\right)$. (b) The thermal Hall conductivity density $\left(\alpha_{x y}^{(\mathrm{PG})}(\mathbf{k}, T, B)\right)$ on the first Brillouin zone in the pseudogap phase at $T=127 \mathrm{~K}, p=9.94 \%$, and $B=0.08$ Tesla. The scale of the plot in (a) is from -0.06 to 0.06 while that in (b) is from -3 to 3 in arbitrary unit. The main contributions to $\left(\sigma_{x y}, \alpha_{x y}\right)$ arise from the density of states corresponding to those portions of the Fermi arc which are not part of the nodal region; there is no contribution from the antinodal region either.

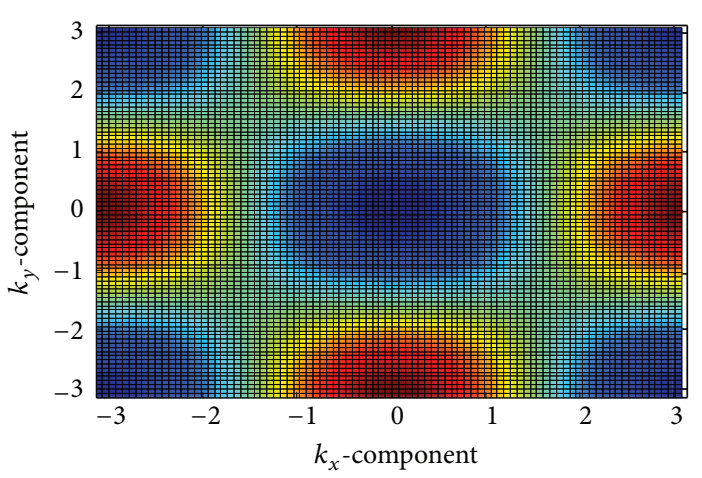

(a)

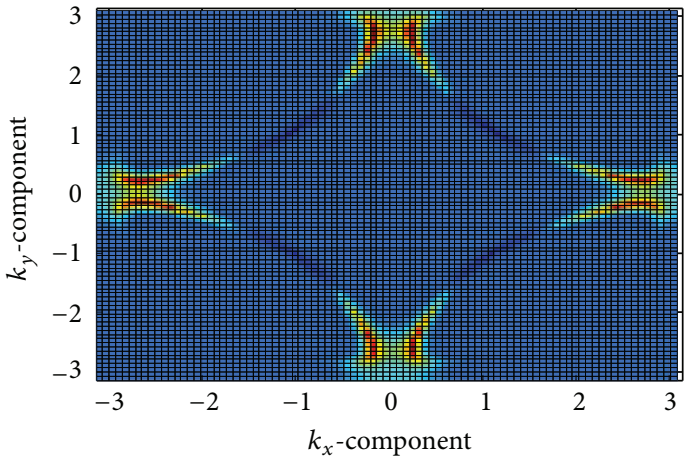

(b)

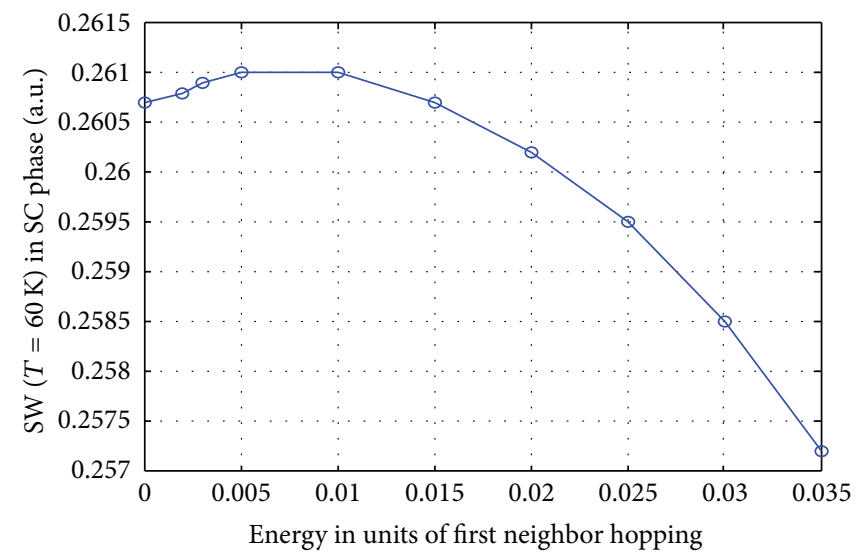

(c)

Figure 14: (a) The contour plot of the density $\sum_{\alpha} A(\mathbf{k}, \omega) \varepsilon_{x x}(\mathbf{k}) n_{k, \alpha}$, where the subscript " $\alpha$ " stands for the conduction band, on the first BZ for the SC phase at $60 \mathrm{~K}$. The gap amplitude $\left(\Delta_{\mathrm{sc}}^{(0)} / t_{1}\right) \sim 0.01$. (b) The contour plot of the density $\sum_{\alpha} A(\mathbf{k}, \omega) \varepsilon_{x x}(\mathbf{k}) n_{k, \alpha}$ on the first BZ for the PG phase at $120 \mathrm{~K}$. The gap amplitude $\left(\Delta_{\mathrm{PG}}^{(0)} / t_{1}\right) \sim 0.07$. There is considerable depletion of the spectral weight density available for pairing in the antinodal region of momentum space in the PG phase compared to the SC phase as could be inferred from the shrinkage of the hot region. (c) A $2 \mathrm{D}$ plot of $F(\omega)$ at $T=60 \mathrm{~K}$ as a function of energy in units of the first neighbor hopping. The integral $F(\omega)$ is a decreasing function for energy larger than that corresponding to the gap amplitude. 


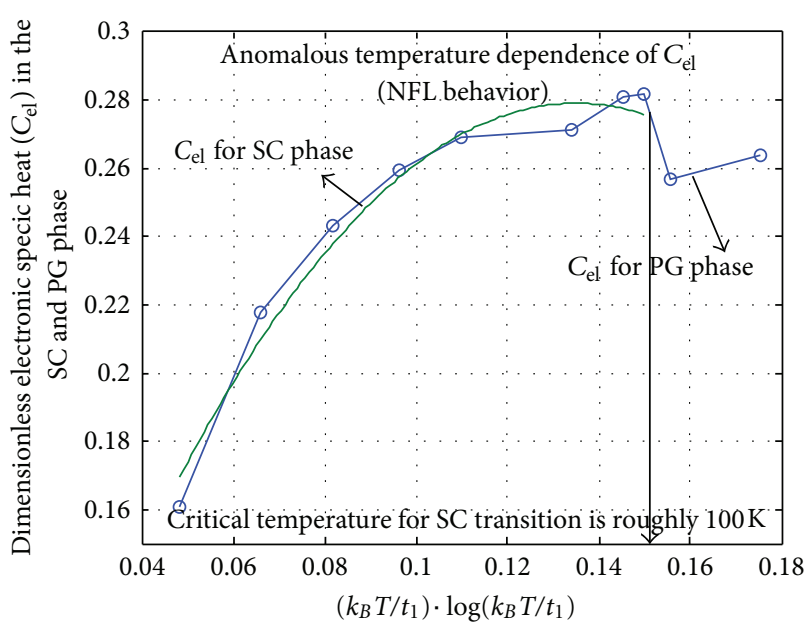

Figure 15: A plot of the dimensionless electronic specific heat $\left(C_{\mathrm{el}}\right)$ in the DSC and DDW phases as a function of $\left[\left(k_{B} T / t_{1}\right) \log \left(k_{B} T / t_{1}\right)\right]$ and a second-degree polynomial fit $\left(C_{\mathrm{el}} \approx-14.5830+\right.$ $\left.3.9355\left[\left(k_{B} T / t_{1}\right) \log \left(k_{B} T / t_{1}\right)\right]-0.0136\left[\left(k_{B} T / t_{1}\right) \log \left(k_{B} T / t_{1}\right)\right]^{2}\right)$. The anomalous temperature dependence of $C_{\mathrm{el}}$ is indicative of the nonFermi liquid (NFL) behavior.

if a problem so requires. For example, the screened plasma frequency $\omega_{p}$, approximated with the plasma edge in the reflectivity $R(\omega)$, may be introduced in place of $\dot{\omega}$ while dealing with the problem of metals. We, however, refrain from doing so as we are not dealing with a similar system.

The longitudinal optical conductivity $\sigma_{x x}(\omega)$ due to an applied field $E_{x}$, in the linear response theory, is given by the frequency-dependent current $j_{x}(\omega)=\sigma_{x x}(\omega) E_{x}(\omega)$. The quantity $j_{x}(\omega)$ may also be expressed in terms the vector potential $A_{x}(\omega)$ of the applied field as $j_{x}(\omega)=\left\langle J_{x}(\omega)\right\rangle+\Pi_{j x, j x}(\omega) A_{x}(\omega)$, where $\Pi_{j x, j x}(\omega)=(i / N) \int_{0}^{\infty} d t e^{i \omega t}\left\langle\left[J_{x}(t), J_{x}(0)\right]\right\rangle$ is the current-current correlation function, and $N$ is the number of $\mathbf{k}$-vectors in the Brillouin zone. Here, $J_{x}(t)$ is the current operator in the interaction representation: $J_{x}(t)=\exp \left(i H_{0} t\right) J_{x} \exp \left(-i H_{0} t\right)$, where $H_{0}$ is the Hamiltonian with $\mathbf{A}=0$. The conical brackets $\langle\cdots\rangle$ stand for an equilibrium average defined with the Hamiltonian $H_{0}$. For simplicity, one may assume the electron self-energy to be q-independent or the vector potential to be positionindependent. It can then be shown that the vertex corrections in the current-current correlation function vanish for $\mathbf{q} \rightarrow 0$, due to the odd parity of the current operator. As in $[32,40]$, the equilibrium average of the current operator $\left\langle J_{x}(\omega)\right\rangle=-S A_{x}(\omega)$, where $S=\left(N^{-1} \sum_{k}^{\prime} \varepsilon_{x x}(\mathbf{k}) n_{k}(T)\right), \varepsilon_{x x}(\mathbf{k})$ is the second derivative with respect to $k_{x}$ of dispersion $\varepsilon(\mathbf{k})$, and $n_{k}(T)$ is the momentum distribution function. The prime symbol in the sum above is to emphasize that only the conduction band contributions need to be taken into account. In view of this result, we obtain $j_{x}(\omega)=\left(-S+\Pi_{j x, j x}(\omega)\right) A_{x}(\omega)$. Now as noted above, $j_{x}(\omega)=\sigma_{x x}(\omega) E_{x}(\omega)$. The vector potential $A_{x}(\omega)$ is related to the field component $E_{x}(\omega)$ by $E_{x}(\omega)=i\left(\omega+i 0^{+}\right) A_{x}(\omega)$.
It follows that $[32,40] \sigma_{x x}(\omega)=\left[-S+\Pi_{j x, j x}(\omega)\right] / i\left(\omega+i 0^{+}\right)$ where the usual spectral form for $\Pi_{j x, j x}(\omega)$ is

$$
\Pi_{j x, j x}(q x, \omega)=\frac{\int\left(d \omega^{\prime} / \pi\right) \chi_{j x, j x}\left(q_{x}, \omega^{\prime}\right)}{\omega^{\prime}-\omega-i 0^{+}} .
$$

Here, the Fourier space current-current correlation function $\chi_{j x, j x}\left(q_{x}, \omega\right)$ is related to the charge correlation function $\chi_{\rho, \rho},\left(q_{x}, \omega\right)$ in the form $\chi_{j x, j x}\left(q_{x}, \omega\right)=\left(\omega^{2} / q_{x}^{2}\right) \chi_{\rho, \rho}\left(q_{x}, \omega\right)$ through the equation of continuity $(\partial \rho(\mathbf{r}, t) / \partial t)+\nabla$. $\mathbf{j}(\mathbf{r}, t)=0$. One may then write $q_{x}^{-2} \int(\omega d \omega / \pi) \chi_{\rho, \rho}\left(q_{x}, \omega\right)=$ $\int(d \omega / \pi \omega) \chi_{j x, j x}\left(q_{x}, \omega\right)$. The integral in the right-hand side of this equation may be shown equal to $S$ as the corresponding left-hand side is

$$
\begin{aligned}
& q_{x}^{-2} \int\left(\frac{\omega d \omega}{\pi}\right) \chi_{\rho, \rho}\left(q_{x}, \omega\right) \\
& \quad=q_{x}^{-2} i \frac{\partial}{\partial t} \int\left(\frac{d \omega}{2 \pi}\right) \exp (-i \omega t) 2 \chi_{\rho, \rho}\left(q_{x}, \omega\right) \\
& =\left.\left(N^{1 / 2} q_{x}\right)^{-2}\left\langle\left[i \frac{\partial}{\partial t} \rho(\mathbf{q}, t), \rho(-\mathbf{q}, 0)\right]\right\rangle\right|_{t=0} \\
& =\left.\left(N^{1 / 2} q_{x}\right)^{-2}\langle[[\rho(\mathbf{q}), H](t), \rho(-\mathbf{q}, 0)]\rangle\right|_{t=0},
\end{aligned}
$$

and the double commutator, in the long wavelength limit, is found to be approximately equal to $S$. We substitute, for $S$, the outcome of this exercise, namely, $\int(d \omega / \pi \omega) \chi_{j x, j x}\left(q_{x}, \omega\right)=$ $S$, in the result $\sigma_{x x}(\omega)=\left[-S+\Pi_{j x, j x}(\omega)\right] / i\left(\omega+i 0^{+}\right)$ above to obtain $\sigma_{x x}(\omega)=\int(d \omega / \pi i \omega) \chi_{j x, j x}(\mathbf{q}, \omega) /\left(\omega+i 0^{+}\right)$. This eventually yields $\operatorname{SW}(T)=N^{-1} \sum_{k}^{\prime} \varepsilon_{x x}(\mathbf{k}) n_{k}(T)$. We note that the elastic scattering by impurities, as discussed in Section 3.2, has not been taken into account in this derivation. We shall assume below, instead, an intrinsic lifetime broadening $\left(\gamma / t_{1}\right) \sim 0.1$ independent of momentum.

As the outline of the derivation above shows, the spectral weight $\mathrm{SW}(T)$ is obtained from the real part of the optical conductivity (OC). We reemphasize that we have taken into account only the carrier contribution from the conduction band to OC and assumed the direction $x$ to be the direction of the current flow when induced by an electromagnetic field in the same direction. For $\hbar=1$, we are, thus, led to

$$
\mathrm{SW}(T)=\left(\frac{\pi e^{2}}{N_{s}}\right) \sum_{k}^{\prime} \varepsilon_{x x}(\mathbf{k}) n_{k}(T) .
$$

We slightly modify (43) replacing the $\mathbf{k}$-summation above by $\int_{-\pi}^{+\pi} d\left(k_{x} a\right) / 2 \pi \int_{-\pi}^{+\pi}\left(d\left(k_{y} a\right) / 2 \pi\right) A(\mathbf{k}, \omega)$, where $A(\mathbf{k}, \omega)$, given below (11), is the density of states (DOSs) or spectral function (SF) obtained using the Matsubara propagators with poles at $\pm E_{\mathbf{k}}^{(U, L)}$. The Fermi energy DOS corresponds to $\omega=0$. The reason for not using (35) giving the DOS with impurity scattering is the fact that in deriving (43) the scattering aspect has not been taken into account. To obtain the SF and SW $(T)$, the coupled gap equations for $i \Delta_{k}$ and $\Delta_{k}^{(\mathrm{sc})}$ together with 


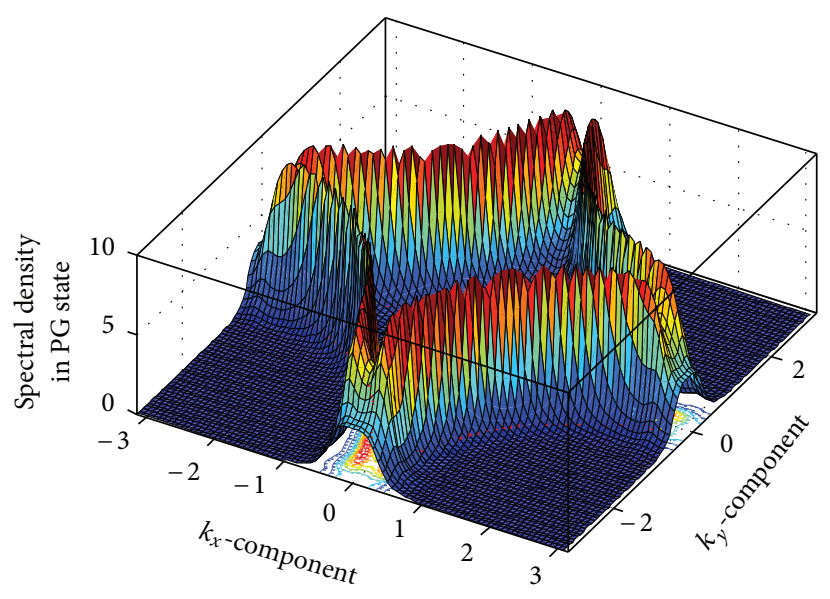

(a)

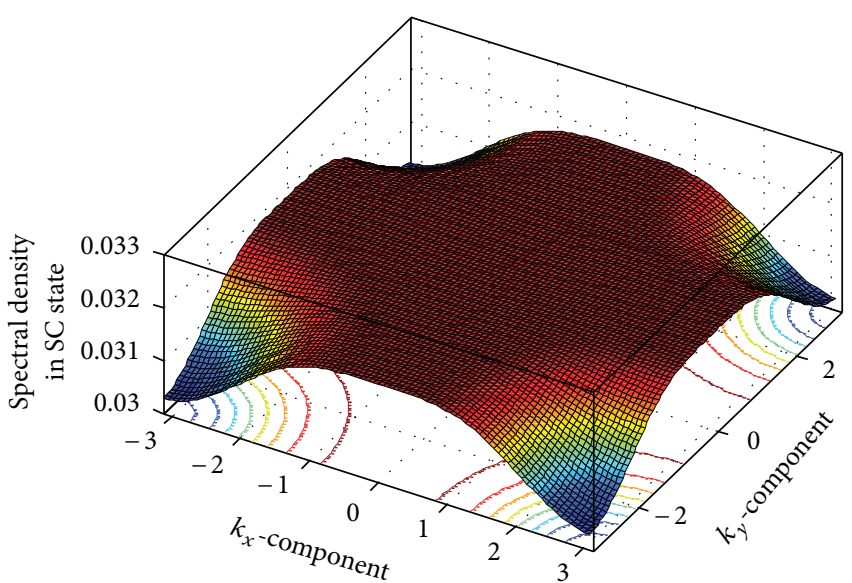

(b)

Figure 16: (a) The plot of the spectral function in the pseudogap phase (doping level 9.94\%) at $\omega=0$ (or, the Fermi energy density of states) on the first Brillouin zone (BZ) for $\left(\mu / t_{1}\right)=-0.0189,\left(\Delta_{0}^{(\mathrm{pg})} / t_{1}\right)=0.0707$, and $\left(\Delta_{0}^{(\mathrm{sc})} / t_{1}\right)=0$. The hopping parameters are $\left(t_{2} / t_{1}\right)=0.3925$ and $\left(t_{3} / t_{1}\right)=0.0005$. (b) The plot of the spectral function in the superconducting phase (doping level 9.94\%) at $\omega=0$ (or, the Fermi energy density of states) on the first Brillouin zone (BZ) for $\left(\mu / t_{1}\right)=-0.00183,\left(\Delta_{0}^{(\mathrm{pg})} / t_{1}\right)=0.024$, and $\left(\Delta_{0}^{(\mathrm{sc})} / t_{1}\right)=0.015$. The hopping parameters are $\left(t_{2} / t_{1}\right)=0.39125$ and $\left(t_{3} / t_{1}\right)=0.0005$. The intrinsic lifetime broadening $\left(\gamma / t_{1}\right)$ has been assumed to be 0.1 .

the equation to determine the chemical potential $(\mu)$ of the fermion number have been solved in a consistent manner with the pinning of the van Hove singularity (vHS) close to $\mu$.

We consider the quantity $F(\omega)=\int_{-\pi}^{+\pi} d\left(k_{x} a\right) /$ $2 \pi \int_{-\pi}^{+\pi} d\left(k_{y} a\right) / 2 \pi \sum_{\alpha} A(\mathbf{k}, \omega) \varepsilon_{x x}(\mathbf{k}) n_{k, \alpha}$, where the subscript " $\alpha$ " stands for the conduction band. In Figures 14(a) and 14(b) above, we have contour-plotted the density $\sum_{\alpha} A(\mathbf{k}, \omega) \varepsilon_{x x}(\mathbf{k}) n_{k, \alpha}$ in the SC phase and the PG phase, respectively, whereas in Figure 14(c) we have plotted the quantity $F(\omega)$ as a function of $\omega$ for $T=60 \mathrm{~K}<T_{c} \sim 100 \mathrm{~K}$. We find that there is considerable depletion of the spectral weight density available for pairing in the antinodal region of momentum space in the PG phase compared to the SC phase as could be inferred from the shrinkage of the hot region in Figure 14(b) in contrast with Figure 14(a). To obtain the data for Figure 14(c), k-integration is necessary. For the integration purpose, we first divide the $\mathrm{BZ}$ into finite number of rectangular patches. We next determine the numerical values corresponding to each of these patches of the momentum-dependent density and sum these values. The sum is then divided by the number of patches. We have generated these values through a surface plot. The gap amplitude at $60 \mathrm{~K}$ is $\left(\Delta_{\mathrm{sc}}^{(0)} / t_{1}\right) \sim 0.01$. The integral $F(\omega)$ is a decreasing function for energy larger than that corresponding to the gap amplitude. This is supportive of the fact that DSC of the hole-doped cuprates corresponds to the strong coupling superconductivity.

\section{Concluding Remarks}

In this communication, we have assumed that the SC order parameter is such that it is linked with an attractive interaction $V\left(\mathbf{k}, \mathbf{k}^{\prime}\right)=-\left|V_{1}\right|\left(\cos k_{x} a-\cos k_{y} a\right)\left(\cos k_{x}^{\prime} a-\cos k_{y}^{\prime} a\right)$, where $V_{1}$ is the coupling strength, that produces the DSC pairing given approximately by $\Delta_{\mathbf{k}}^{(\mathrm{SC})}=-\sum_{\mathbf{k}^{\prime}} V\left(\mathbf{k}, \mathbf{k}^{\prime}\right) \Delta_{\mathbf{k}^{\prime}}^{(\mathrm{SC})} \times$ $\left[{\sqrt{\varepsilon_{\mathbf{k}^{\prime}}^{2}+\Delta_{\mathbf{k}^{\prime}}^{2}+\Delta_{\mathbf{k}^{\prime}}^{(\mathrm{SC}) 2}}}^{-1}\right.$. We have assumed that a combination of e-e and e-bm interactions will lead to a $d$-wave gap $\Delta_{k}^{(\mathrm{SC})}$. It must be clarified that our effort to obtain the solution of the gap equation has not been ambitious enough to aim at attempting the settlement of the long debated issue [59] whether the pairing interactions are of purely electronic and (or) electron-bosonic mode origin. In fact, assuming that these agents jointly or in a mutually exclusive manner provide us the requisite pairing interactions, we bypass this vital issue.

The angle-resolved photoemission spectroscopy (ARP ES), which is a valuable tool in the toolkit of experimentalists for studying the PG phase, shows Fermi arcs [15-20], below the characteristic temperature $T^{*}$, centered along the zone diagonal instead of the expected Fermi surface (FS). Though a general consensus exists about the existence of the Fermi arcs, there is again a debate on their main characteristics which revolves around the following issues. Some authors [15-17] report that the Fermi arcs are linked to the preformed pair scenario. Near the antinode there exists a single gap, nearly independent of temperature, and in the superconducting state the gap follows the expected $d$-wave behavior along the FS. In contrast, other group of authors [18-20,24] claim that arcs are associated to a pairing which is distinct from that corresponding to SC. These theoretical and experimental studies carried out so far have been able to keep the interest alive for solving this puzzle. In this communication, as already mentioned, our focal point is the latter view. While the singleparticle excitation spectrum of the DDW state involves the usual fermionic particle $(p)$ and hole $(h)$ pairing/mixing in the singlet channel with an associated energy scale of roughly 
$20 \mathrm{meV}$, the coexisting and competing DDW and DSC states correspond to the well-known Bogoliubov quasiparticles with an associated energy scale of nearly $10 \mathrm{meV}$. Most importantly, from our viewpoint, the passage to the PG state from the normal state, upon cooling at a fixed underdoping level, corresponds to a nonsharp thermal transition rather than a smooth crossover as is suggested by the authors who advocate the preformed pairing scenario. The PG phase, as we have shown in Section 3.3, is characterized by the nonFermi liquid (NFL) behavior. This NFL behavior persists in the superconducting DDW phase.

We have shown above (see Figure 15) a plot of the dimensionless electronic specific heat $\left(C_{\mathrm{el}}\right)$ in the SC and PG phases as a function of $\left[\left(k_{B} T / t_{1}\right) \log \left(k_{B} T / t_{1}\right)\right]$ and a second-degree polynomial fit. The details of this part of the investigation will be reported elsewhere. The anomalous temperature dependence of $C_{\mathrm{el}}$ in Figure 15 is indicative of the non-Fermi liquid (NFL) behavior persisting even in the $\mathrm{DDW}+\mathrm{DSC}$ phase. The discontinuity in $\mathrm{C}_{\mathrm{el}}$ occurs roughly at $T \approx 100 \mathrm{~K}$ due to the passage from the PG phase to SC phase at the fixed doping level 9.94\%. This allows us to infer that the critical temperature $\left(T_{c}\right)$ of the superconducting transition for a generic hole-doped cuprate is approximately $100 \mathrm{~K}$.

We have mentioned below (11) that the spectral function $A(\mathbf{k}, \omega)$ in the DDW + DSC phase is given by a sum of $\delta$ functions at the quasiparticle energies:

$$
A(\mathbf{k}, \omega)=2 \pi \sum_{j= \pm 1}\left[u_{\mathbf{k}}^{(j)^{2}} \delta\left(\omega+j E_{\mathbf{k}}^{(U)}\right)+v_{\mathbf{k}}^{(j)^{2}} \delta\left(\omega+j E_{\mathbf{k}}^{(L)}\right)\right]
$$

We simply replace the $\delta$ functions here by Lorentzians with an assumed intrinsic lifetime broadening $\left(\gamma / t_{1}\right) \sim 0.1$ as was done in Section 2. From the expression for spectral density, putting put $\omega=0$, we then obtain

$$
\begin{aligned}
\rho_{\mathrm{Fermi}}(\mathbf{k})= & \left(\frac{1}{2 \pi \rho_{0}}\right) \sum_{j=( \pm)}\left[u_{\mathbf{k}}^{(j)^{2}}\left(\frac{\gamma}{t_{1}}\right)\right. \\
& \times\left[E_{\mathbf{k}}^{(U) 2}+\left(\frac{\gamma}{t_{1}}\right)^{2}\right]^{-1}+v_{\mathbf{k}}^{(j)}\left(\frac{\gamma}{t_{1}}\right) \\
& \times\left[E_{\mathbf{k}}^{(L) 2}+\left(\frac{\gamma}{t_{1}}\right)^{2}\right]^{-1} .
\end{aligned}
$$

This leads to the usual Fermi arc picture on the first Brillouin zone in the PG phase and nearly a constant in the SC phase. In Figure 16, we have depicted the plots of the spectral density in the PG and SC phases assuming the abovementioned value of the intrinsic lifetime broadening. The plot in Figure 16(b) validates the approximation of the Fermi energy density of states (DOS) by a constant we made while seeking solutions of the gap equations analytically in the SC phase in (17).

The authors, who link PG to preformed pair scenario, advocate the existence and relevance of superconducting fluctuations (SCF) with short correlation lengths in the underdoped cuprates for transport properties. In particular, the survival of a large Nernst signal $[3,4]$ up to temperatures much larger than the superconducting transition temperature $T_{c}$ in the pseudogap region has been interpreted as the evidence for such SCFs. The fluctuations are supposed to correspond to vortex-like phase fluctuations with a Beresinkii-Kosterlitz-Thouless (BKT) character due to the quasi-two-dimensional (2D) nature of the system [4]. In our mean field approach neither there is such superconductingfluctuations nor there are vortices in the normal state, as in the preformed pair scenario, supporting a Nernst signal. In a future work, our aim would be to look into this aspect.

\section{References}

[1] V. J. Emery and S. A. Klvelson, "Importance of phase fluctuations in superconductors with small superfluid density," Nature, vol. 374, no. 6521, pp. 434-437, 1995.

[2] M. Franz, Z. Tešanović, and O. Vafek, " $\mathrm{QED}_{3}$ theory of pairing pseudogap in cuprates: from $d$-wave superconductor to antiferromagnet via an algebraic fermi liquid," Physical Review B, vol. 66, no. 5, Article ID 054535, 2002.

[3] Y. Wang, L. Li, and N. P. Ong, "Nernst effect in high- $T_{c}$ superconductors," Physical Review B, vol. 73, no. 2, Article ID 024510, 2006.

[4] L. Li, Y. Wang, S. Komiya et al., "Diamagnetism and Cooper pairing above $T_{c}$ in cuprates," Physical Review B, vol. 81, no. 5, Article ID 054510, 2010.

[5] A. Kanigel, U. Chatterjee, M. Randeria et al., "Evidence for pairing above the transition temperature of cuprate superconductors from the electronic dispersion in the pseudogap phase," Physical Review Letters, vol. 101, no. 13, Article ID 137002, 2008.

[6] O. Yuli, I. Asulin, Y. Kalcheim, G. Koren, and O. Millo, "Proximity-Induced Pseudogap: Evidence for Preformed Pairs," Physical Review Letters, vol. 103, no. 19, Article ID 197003, 2009.

[7] A. Levchenko, M. R. Norman, and A. A. Varlamov, "Nernst effect from fluctuating pairs in the pseudogap phase of the cuprates," Physical Review B, vol. 83, no. 2, Article ID 020506, 2011.

[8] S. Hüfner, M. A. Hossain, A. Damascelli, and G. A. Sawatzky, “Two gaps make a high-temperature superconductor?" Reports on Progress in Physics, vol. 71, no. 6, Article ID 062501, 2008.

[9] S. Chakravarty, R. B. Laughlin, D. K. Morr, and C. Nayak, "Hidden order in the cuprates," Physical Review B, vol. 63, no. 9, Article ID 094503, pp. 945031-9450310, 2001.

[10] I. Dimov, P. Goswami, X. Jia, and S. Chakravarty, "Competing order, Fermi surface reconstruction, and quantum oscillations in underdoped high-temperature superconductors," Physical Review B, vol. 78, no. 13, Article ID 134529, 2008.

[11] S. Chakravarty, "Quantum oscillations and key theoretical issues in high temperature superconductors from the perspective of density waves," Reports on Progress in Physics, vol. 74, no. 2, Article ID 022501, 2011.

[12] J.-X. Li, C.-Q. Wu, and D.-H. Lee, "Checkerboard charge density wave and pseudogap of high- $T_{c}$ cuprate," vol. 74, no. 18, Article ID 184515, 2006.

[13] A. Levchenko, T. Micklitz, M. R. Norman, and I. Paul, "Transport implications of Fermi arcs in the pseudogap phase of the 
cuprates," Physical Review B, vol. 82, no. 6, Article ID 060502, 2010.

[14] A. Damascelli, Z. Hussain, and Z. X. Shen, "Angle-resolved photoemission studies of the cuprate superconductors," Reviews of Modern Physics, vol. 75, no. 2, pp. 473-541, 2003.

[15] A. Kanigel, M. R. Norman, M. Randeria et al., "Evolution of the pseudogap from Fermi arcs to the nodal liquid," Nature Physics, vol. 2, no. 7, pp. 447-451, 2006.

[16] A. Kanigel, U. Chatterjee, M. Randeria et al., "Protected nodes and the collapse of fermi arcs in high- $T_{c}$ cuprate superconductors," Physical Review Letters, vol. 99, no. 15, Article ID 157001, 2007.

[17] M. Shi, J. Chang, S. Pailhés et al., "Coherent $d$-Wave superconducting gap in underdoped $\mathrm{La}_{2-x} \mathrm{Sr}_{x} \mathrm{CuO}_{4}$ by angle-resolved photoemission spectroscopy," Physical Review Letters, vol. 101, no. 4, Article ID 047002, 2008.

[18] K. Terashima, H. Matsui, T. Sato, T. Takahashi, M. Kofu, and K. Hirota, "Anomalous momentum dependence of the superconducting coherence peak and its relation to the pseudogap of $\mathrm{La}_{1.85} \mathrm{Sr}_{0.15} \mathrm{CuO}_{4}$," Physical Review Letters, vol. 99, no. 1, Article ID 017003, 2007.

[19] T. Yoshida, M. Hashimoto, S. Ideta et al., "Universal versus material-dependent two-gap behaviors of the high- $T_{c}$ cuprate superconductors: Angle-resolved photoemission study of $\mathrm{La}_{2-x} \mathrm{Sr}_{x} \mathrm{CuO}_{4}$," Physical Review Letters, vol. 103, no. 3, Article ID 037004, 2009.

[20] T. Kondo, R. Khasanov, T. Takeuchi, J. Schmalian, and A. Kaminski, "Competition between the pseudogap and superconductivity in the high- $T_{c}$ copper oxides," Nature, vol. 457, no. 7227, pp. 296-300, 2009.

[21] D. Z. Liu and K. Levin, "Long range Coulomb effects in the cuprates: a possible alternative route to $d$-wave superconductivity," Physica C, vol. 275, no. 1-2, pp. 81-86, 1997.

[22] P. Kotetes and G. Varelogiannis, "Meissner effect without superconductivity from a chiral $d$-density wave," Physical Review B, vol. 78, no. 22, Article ID 220509, 2008.

[23] P. Kotetes and G. Varelogiannis, "Chirality induced tilted-hill giant Nernst signal," Physical Review Letters, vol. 104, no. 10, Article ID 106404, 2010.

[24] M. Hashimoto, R.-H. He, K. Tanaka et al., "Particle-hole symmetry breaking in the pseudogap state of Bi2201," Nature Physics, vol. 6, no. 6, pp. 414-418, 2010.

[25] R.-H. He, M. Hashimoto, H. Karapetyan et al., "From a singleband metal to a high-temperature superconductor via two thermal phase transitions," Science, vol. 331, no. 6024, pp. 1579-1583, 2011.

[26] K. Tanaka, T. Yoshida, A. Fujimori et al., "Effects of nextnearest-neighbor hopping $\mathrm{t}^{\prime}$ on the electronic structure of cuprate superconductors," Physical Review B, vol. 70, no. 9, Article ID 092503, 2004.

[27] J. Chang, N. Doiron-Leyraud, F. Laliberté et al., "Nernst effect in the cuprate superconductor $\mathrm{YBa}_{2} \mathrm{Cu}_{3} \mathrm{O}_{y}$ : broken rotational and translational symmetries," Physical Review B, vol. 84, no. 1, Article ID 014507, 2011.

[28] C. Zhang, S. Tewari, V. M. Yakovenko, and S. Das Sarma, "Anomalous Nernst effect from a chiral $d$-density-wave state in underdoped cuprate superconductors," Physical Review B, vol. 78, no. 17, Article ID 174508, 2008.

[29] S. Tewari, C. Zhang, V. M. Yakovenko, and S. Das Sarma, “Timereversal symmetry breaking by a $(d+i d)$ density-wave state in underdoped cuprate superconductors," Physical Review Letters, vol. 100, no. 21, Article ID 217004, 2008.

[30] J. Xia, E. Schemm, G. Deutscher et al., "Polar kerr-effect measurements of the high-temperature $\mathrm{YBa}_{2} \mathrm{Cu}_{3} \mathrm{O}_{6+x}$ superconductor: evidence for broken symmetry near the pseudogap temperature," Physical Review Letters, vol. 100, no. 12, Article ID 127002, 2008.

[31] A. Kaminski, M. Randeria, J. C. Campuzano et al., "Renormalization of spectral line shape and dispersion below $T_{c}$ in $\mathrm{Bi}_{2} \mathrm{Sr}_{2} \mathrm{CaCu}_{2} \mathrm{O}_{8+\delta}$, Physical Review Letters, vol. 86, no. 6, pp. 1070-1073, 2001.

[32] A. J. Millis, "Optical conductivity and correlated electron physics," in Strong Interactions in Low Dimensions, D. Baeriswyl and L. DeGiorgi, Eds., Springer, Berlin, Germany, 2004.

[33] E. Khatami, K. Mikelsons, D. Galanakis et al., "Quantum criticality due to incipient phase separation in the two-dimensional Hubbard model," Physical Review B, vol. 81, no. 20, Article ID 201101, 2010.

[34] C. Presura, D. Van Der Marel, A. Damascelli, and R. K. Kremer, "Low-temperature ellipsometry of $\alpha^{\prime}-\mathrm{NaV}_{2} \mathrm{O}_{5}$," Physical Review $B$, vol. 61, no. 23, pp. 15762-15765, 2000.

[35] M. R. Norman, A. V. Chubukov, E. Van Heumen, A. B. Kuzmenko, and D. Van Der Marel, "Optical integral in the cuprates and the question of sum-rule violation," Physical Review B, vol. 76, no. 22, Article ID 220509, 2007.

[36] D. Baeriswyl, J. Carmelo, and A. Luther, "Correlation effects on the oscillator strength of optical absorption: sum rule for the one-dimensional Hubbard model," Physical Review B, vol. 33, no. 10, pp. 7247-7248, 1986.

[37] D. Baeriswyl, C. Gros, and T. M. Rice, "Landau parameters of almost-localized Fermi liquids," Physical Review B, vol. 35, no. 16, pp. 8391-8395, 1987.

[38] H. J. A. Molegraaf, C. Presura, D. Van der Marel, P. H. Kes, and M. Li, "Superconductivity-induced transfer of in-plane spectral weight in $\mathrm{Bi}_{2} \mathrm{Sr}_{2} \mathrm{CaCu}_{2} \mathrm{O}_{8+\delta}$, Science, vol. 295, no. 5563, pp. 2239-2241, 2002.

[39] A. F. Santander-Syro, R. P. S. M. Lobo, N. Bontemps, Z. Konstantinovic, Z. Z. Li, and H. Raffy, "Pairing in cuprates from high-energy electronic states," Europhysics Letters, vol. 62, no. 4, pp. 568-574, 2003.

[40] D. Bergeron, V. Hankevych, B. Kyung, and A.-M. S. Tremblay, "Optical and dc conductivity of the two-dimensional Hubbard model in the pseudogap regime and across the antiferromagnetic quantum critical point including vertex corrections," Physical Review B, vol. 84, no. 8, Article ID 085128, 2011.

[41] J. P. F. Leblanc, J. P. Carbotte, and E. J. Nicol, "Effects of a particle-hole asymmetric pseudogap on Bogoliubov quasiparticles," Physical Review B, vol. 83, no. 18, Article ID 184506, 2011.

[42] K.-Y. Yang, T. M. Rice, and F.-C. Zhang, "Phenomenological theory of the pseudogap state," Physical Review B, vol. 73, no. 17, Article ID 174501, 2006.

[43] K.-Y. Yang, H.-B. Yang, P. D. Johnson, T. M. Rice, and F.-C. Zhang, "Quasiparticles in the pseudogap phase of underdoped cuprate," Europhysics Letters, vol. 86, no. 3, Article ID 37002, 2009.

[44] J. Kokalj and P. Prelovšek, "Breakdown of the Luttinger sum rule within the Mott-Hubbard insulator," Physical Review B, vol. 78, no. 15, Article ID 153103, 2008.

[45] A. Hackl and M. Vojta, "Nernst-effect anisotropy as a sensitive probe of Fermi-surface distortions from electron-nematic 
order," Physical Review B, vol. 80, no. 22, Article ID 220514 , 2009.

[46] A. Karl Gschneidner, Handbook on the Physics and Chemistry of Rare Earths, vol. 34, 2005.

[47] L. P. Kadanoff and G. Baym, Quantum Statistical Mechanics, vol. 14, Benzamin, New York, NY, USA, 1962.

[48] P. Goswami, "Some useful relations to derive the thermodynamic potentials of Fermi and Bose systems using spectralweight functions," Physical Review B, vol. 49, no. 3, pp. 1600-1607, 1994.

[49] A. Pushp, C. V. Parker, A. N. Pasupathy et al., "Extending universal nodal excitations optimizes superconductivity in $\mathrm{Bi}_{2} \mathrm{Sr}_{2} \mathrm{CaCu}_{2} \mathrm{O}_{8+\delta}$, Science, vol. 324, no. 5935, pp. 1689-1693, 2009.

[50] J. C. Campuzano, H. Ding, M. R. Norman et al., "Direct observation of particle-hole mixing in the superconducting state by angle-resolved photoemission," Physical Review B, vol. 53, no. 22, pp. R14737-R14740, 1996.

[51] H. Matsui, T. Sato, T. Takahashi et al., "BCS-like Bogoliubov quasiparticles in high- $T_{c}$ superconductors observed by angleresolved photoemission spectroscopy," Physical Review Letters, vol. 90, no. 21, pp. 2170021-2170024, 2003.

[52] H.-B. Yang, J. D. Rameau, P. D. Johnson, T. Valla, A. Tsvelik, and G. D. Gu, "Emergence of preformed Cooper pairs from the doped Mott insulating state in $\mathrm{Bi}_{2} \mathrm{Sr}_{2} \mathrm{CaCu}_{2} \mathrm{O}_{8+\delta}$," Nature, vol. 456, no. 7218, pp. 77-80, 2008.

[53] G. Rickayzen, Green'S Functions and Condensed Matter, chapter 4, Academic Press, London, UK, 1980.

[54] A. Hackl, M. Vojta, and S. Sachdev, "Quasiparticle Nernst effect in stripe-ordered cuprates," Physical Review B, vol. 81, no. 4, Article ID 045102, 2010.

[55] N. Doiron-Leyraud, C. Proust, D. LeBoeuf et al., "Quantum oscillations and the Fermi surface in an underdoped high- $T_{c}$ superconductor," Nature, vol. 447, no. 7144, pp. 565-568, 2007.

[56] D. LeBoeuf, N. Doiron-Leyraud, J. Levallois et al., "Electron pockets in the Fermi surface of hole-doped high- $T_{c}$ superconductors," Nature, vol. 450, no. 7169, pp. 533-536, 2007.

[57] A. F. Bangura, J. D. Fletcher, A. Carrington et al., "Small fermi surface pockets in underdoped high temperature superconductors: observation of Shubnikov-de Haas oscillations in YBa2Cu4O8," Physical Review Letters, vol. 100, no. 4, Article ID 047004, 2008.

[58] S. Riggs, J. Betts, S. Sebastian et al., "Quantum oscillations in the specific heat of ultraclean YBCO in 45T magnetic fields," in APS March meeting, vol. 54, abstract L33-00004, no. 1, Pittsburgh, Pa, USA, 2009.

[59] P. W. Anderson, "Is there glue in cuprate superconductors?" Science, vol. 316, no. 5832, pp. 1705-1707, 2007. 

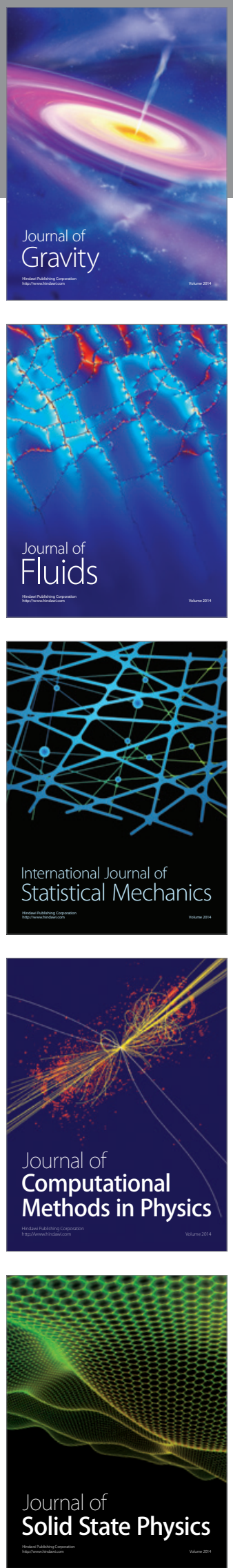

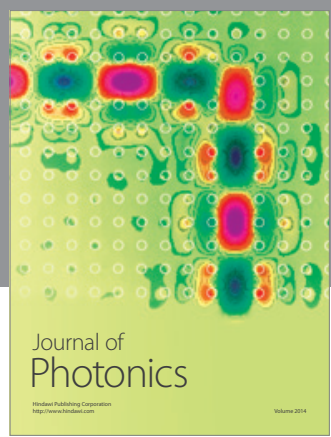

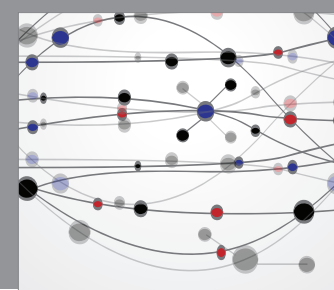

The Scientific World Journal

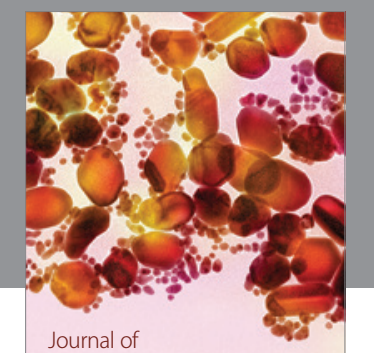

Soft Matter
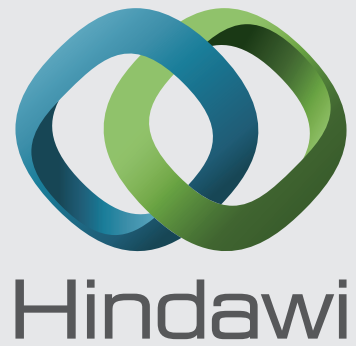

Submit your manuscripts at

http://www.hindawi.com
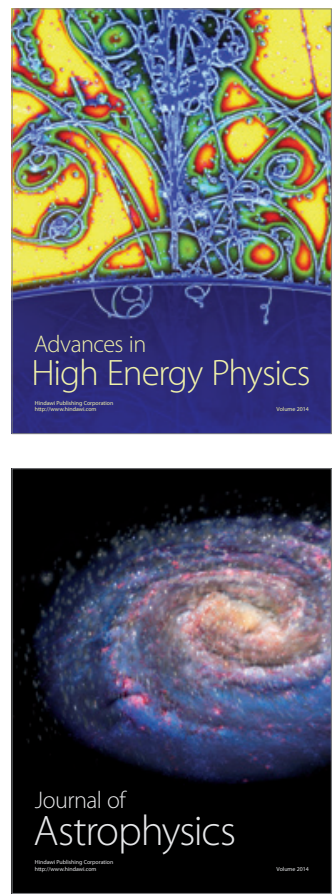
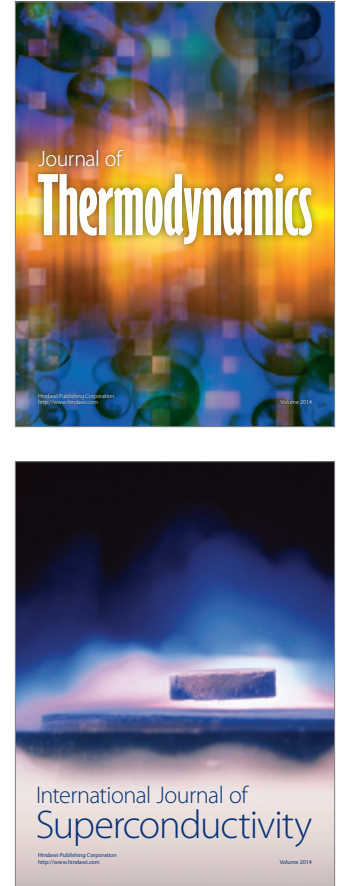
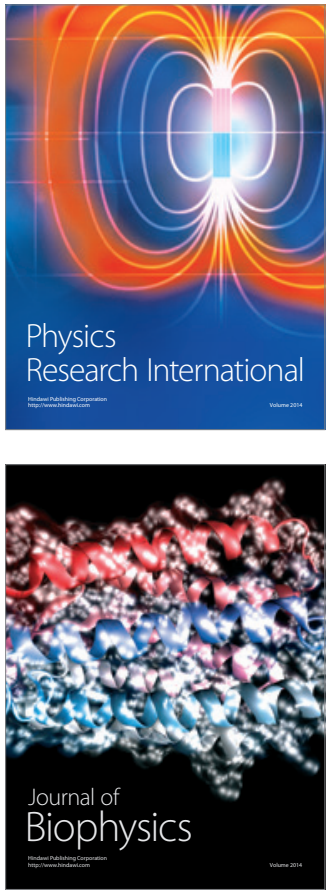
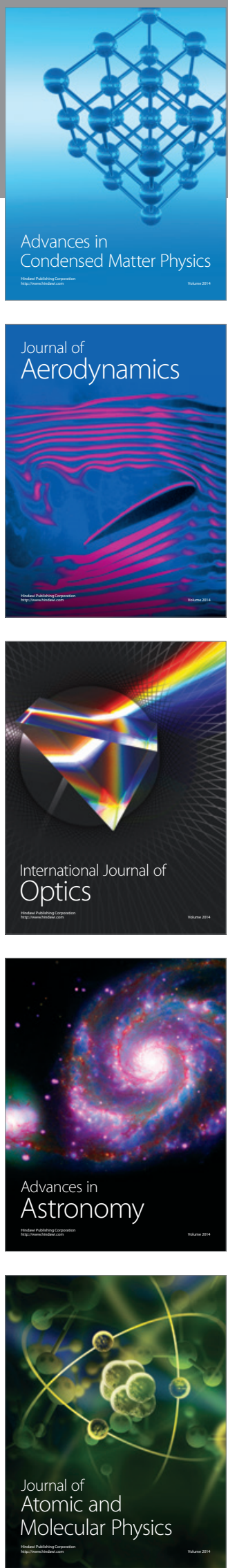